\title{
Overhead Performance Tradeoffs-A Resource Allocation Perspective
}

\author{
Jie Ren, Bradford D. Boyle, Student Member, IEEE, Gwanmo Ku, Student Member, IEEE, \\ Steven Weber, Senior Member, IEEE, and John MacLaren Walsh, Member, IEEE
}

\begin{abstract}
A key aspect of many resource allocation problems is the need for the resource controller to compute a function, such as the max or arg max, of the competing users metrics. Information must be exchanged between the competing users and the resource controller in order for this function to be computed. In many practical resource controllers the competing users' metrics are communicated to the resource controller, which then computes the desired extremization function. However, in this paper it is shown that information rate savings can be obtained by recognizing that controller only needs to determine the result of this extremization function. If the extremization function is to be computed losslessly, the rate savings are shown in most cases to be at most 2 bits independent of the number of competing users. Motivated by the small savings in the lossless case, simple achievable schemes for both the lossy and interactive variants of this problem are considered. It is shown that both of these approaches have the potential to realize large rate savings, especially in the case where the number of competing users is large. For the lossy variant, it is shown that the proposed simple achievable schemes are in fact close to the fundamental limit given by the rate distortion function.
\end{abstract}

\section{Index Terms}

Distributed function computation, extremization, rate distortion, scalar quantization, interactive communication, resource allocation

\section{INTRODUCTION}

In this paper we consider a problem in which series of $N$ users have access to independent sequences $\boldsymbol{X}_{i}=$ $\left[X_{i, s} \mid s \in\{1,2, \ldots\},\right], i \in\{1, \ldots, N\}$ of independent and identically distributed observations $X_{i, s}$ from a known distribution on a set $\mathcal{X} \subset \mathbb{R}^{+}$, a subset of the non-negative real numbers. The users compress their observations for transmission to a chief estimating officer (CEO) that wishes to know for each element in the sequence:

1) the largest observation, i.e. $\max _{i \in\{1, \ldots, N\}} X_{i, s}$ for each $s$;

This research has been supported by the Air Force Research Laboratory under agreement number FA9550-12-1-0086. The U.S. Government is authorized to reproduce and distribute reprints for Governmental purposes notwithstanding any copyright notation thereon.

The authors are with the Department of Electrical and Computer Engineering, Drexel University, Philadelphia, PA USA. The contact author is John MacLaren Walsh (email: jwalsh@ece.drexel.edu)

Preliminary results were reported at CISS 2014 [1], |2]. 


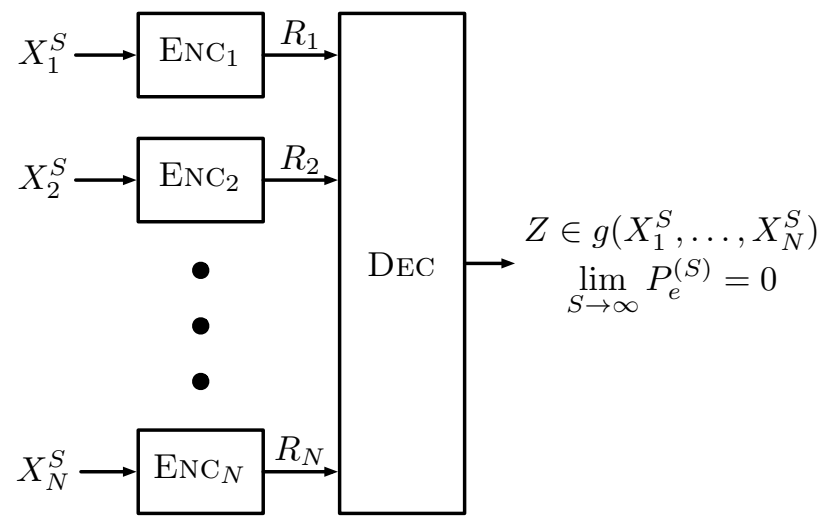

(a)

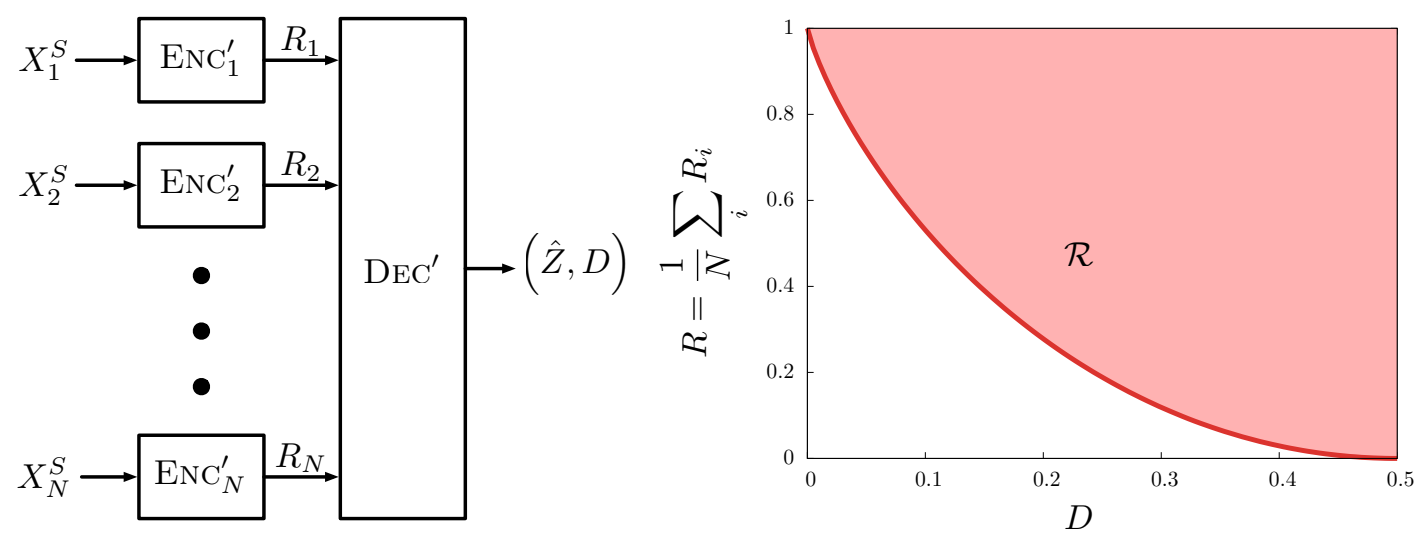

(b)

Fig. 1. Model block diagrams

\begin{tabular}{|c|c|c|c|c|}
\hline & & $\max$ & $\arg \max$ & $(\max , \arg \max )$ \\
\hline 1 & OFDMA resource allocation & anycasting & rateless PHY layer & traditional AMC PHY layer \\
\hline 2 & economics & asset pricing & asset allocation & sealed-bid first-price auctions \\
\hline 3 & sensor network/intrusion detection & is there an intruder & where is the intruder & is there an intruder $\&$ where is the intruder \\
\hline
\end{tabular}

TABLE I

EXAMPLE APPLICATION OF INDIRECT EXTREMAL VALUE COMPUTATION

2) a source having the largest observation, i.e. a single member of $\arg \max _{i \in\{1, \ldots, N\}} X_{i, s}$ for each $s$, or;

3) both the largest observation and the user that having the largest observation.

We refer the three cases as the max problem, the arg max problem, and the (max, arg max) problem respectively. Although we present all our results terms of max, similar results will hold for the corresponding minimization problems. 


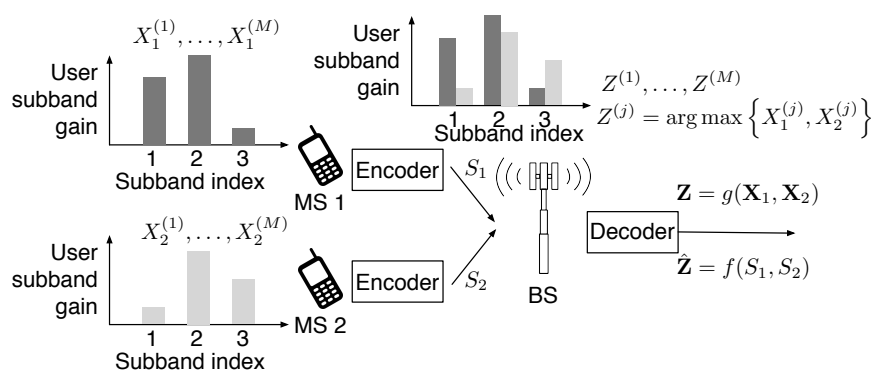

Fig. 2. The BS wishes to compute the index (arg max) of the user with the largest gain on each subband. The users encode their local gains across subbands using SQs.

This generic indirect extremal value computation problem finds examples in several fields; Table 1 lists a few of these. We consider three in more detail here:

Example 1 (orthogonal frequency-division multiple access (OFDMA) resource allocation). Rateless coding, also known as fixed-to-variable coding [3], can achieve performance close to the channel capacity without requiring the explicit feedback of channel state information and use of adaptive modulation and coding in a single user system [4], [5]. These schemes operate by enabling the block length (in channel uses) for the modulation and coding to stretch or shrink based on the received channel quality in a manner that closely resembles H-ARQ. Rather than feeding back channel quality, the receiver only needs to indicate when it has successfully decoded the transmitted message, which it learns through an outer error detection code. In a multiuser OFDMA system, the base station (BS) needs to assign mobile stations (MSs) to subblocks of channles, even when a rateless code used (Fig. 2). If the BS wishes to maximize the sum-rate across users, the uplink feedback from the MS only needs to enable the basestation to determine which MS has the best channel. The BS does not need to know the actual channel gain/capacity. Once the BS has decided which user to schedule on a particular collection of subbands, it must signal this resource decision on the downlink as overhead control information in addition to the data to be transmitted to the user itself. These resource decisions control information, along with the MS's feedback, result in control overheads that are surprisingly large- the control overheads account for $\approx 25-30 \%$ of all downlink transmission in the LTE standard [6].

Anycasting is transmission scheme whereby a sender wishes to send a message to a group of potential receivers and ensure that it is received by at least one receiver [7]. This is contrasted with broadcasting, where every receiver is required to receive the message. In this context, the CEO is the BS and the sourcess at the different receivers are the channel gains/capacities on the downlinks. The BS needs to know the largest channel capacity in order to select an approriate transmission rate. Replacing max with min, this setup becomes a broadcasting problem. By knowing the smallest channel capacity, the BS can select a rate that ensures its message is received by all of the users.

Traditional adaptive modulation and coding (AMC) [8] proceeds by first defining a finite collection of codes and 
modulation schemes associated with different information rates $r_{k}$ measured in bits per channel use. The index $k \in\{1, \ldots, K\}$ indicating which scheme to use is called the modulation and coding scheme (MCS) index. The receiver measures the channel quality using reference or training signals, or pilots, and determines the information rate among this finite set corresponding to a modulation and coding scheme achieving a given target probability of error. The associated index $k$, or some quantization of it, is then fed back to the transmitter under the label channel quality indicator (CQI). The transmitter then takes into consideration factors such as the amount of data waiting to be sent to the various receivers associated with it and their necessary quality of service, then selects the modulation and coding scheme to use when transmitting to them.

Example 2 (Economics). When a seller has a commodity that it wishes to sell, it sets the price with respect to the market [9]. If the seller wants to ensure that it does not price it self out of the market, it would want to compute the max of the individual valuations of a representative sample of the market. Conversely, if the seller wants to undercut its competition it would need to compute the min of the competitor's prices.

In many situations, goods should be allocated/distributed to users based on their "need" or expected derived utility [10]. For example, need-based financial aid for higher education. In this scenario, the entity distributing the goods would only need to calculate the individual with the largest expected derived utility (i.e., the $\arg \max$ ).

We think of the CEO selling a good through an auction to a set of independent buyers. In a sealed-bid first-price auction, the buyers submit bids in "sealed" envelopes and the highest bidder is the winner, paying their bid amount [11]. The auctioneer has no need for recovering this bids of the other users.

Example 3 (Sensor network/intrusion detection [12]). A collection of sensor nodes are monitoring an area large enough that individual sensor readings are independent. As a very simple model, we can take the local sensor outputs to be binary: 0 if no intruder is present, 1 if an intruder is present. Computing the arg max determines where an intruder is (if in fact there is one); computing themax sensor reading determines if an intruder is present but not where, and; computing both determines if and where an intruder is.

The remainder of the paper is organized as follows: In Section [I] we review the existing literature concerning fundamental limits and achievable schemes for the non-interactive lossless and lossy estimation. We also review literature for the interactive variant of this problem where the users and CEO are allowed to interactively communicate over multiple rounds. Next we formalize the mathematical model for this problem and propose natural distortion measures in Section III In Section IV] we derive the fundamental limit on rate to estimate losslessly (in the usual Shannon sense) and propose a scheme to achieve this limit. We observe that the rate savings compared with the source recovery (i.e. Slepian-Wolf (SW) [13]) are not large. In Section $\mathrm{V}$, we consider the same problem, but allow the CEO to estimate the function in a lossy manner. We compute the rate-distortion function numerically (Section $\mathrm{V}-\mathrm{A}$ ) and compare it with an achievable scheme based on scalar quantization (Section $\mathrm{V}-\mathrm{B}$ \& Section $\mathrm{V}-\mathrm{C}$ ). Finally in Section VI] we consider interactive communications [14] between the CEO and the users. We propose interactive schemes in which the CEO will losslessly determine the max, arg max or the pair. For both the one-shot 
lossy and interactive lossless case, we show the rate saving can be substantial.

\section{RELATED WORK}

We first review some previous results of the CEO problem under both lossless and lossy setup to understand the fundamental limits of the rate region. We then review some results about quantization design which help us to give achievable schemes for the extremization problems we are interested in.We will also cover the lossless interactive communication results which allows multi-round communications. In this paper, we consider aymptotically lossless, lossy (i.e., rate-distortion), and interactive limits for the problem of computing the functions of interest. In this section we review the literature for the different approaches to the problem as well as the literature on quantization-based achievable schemes.

\section{A. Related Work-Lossless}

The two-terminal function computation with side information problem has been considered in [15] and [16] where two terminals (transmitter and receiver) each contain a source and the receiver wants to decode a function of the two sources. Earlier work by Witsenhausen considered the problem of minimizing the encoder's alphabet size with the constraint that the computation needs to be zero-error [15]. He showed the minimum alphabet size is related to the chromatic number of the characteristic graph of the source. Orlitsky et al. considered a similar problem setup, but instead of zero-error they allowed an asymptotically small probability of error [16]. With this assumption, they showed the fundamental limit on rate is the graph entropy of the characteristic graph. The distributed function computation problem has been considered in [17] and [18] where the problem is under the CEO setup [19] where the CEO wants to compute a function of the sources from two or more users. Doshi et al. gave the rate region to the problem under a constraint that they term a "zig-zag" condition [18]. They showed that any achievable rate point can be realized by graph coloring at each user and SW encoding the colors. Sefidgaran et al. derived inner and outer bounds to the rate region under for a class of tree structured networks, which includes the classical CEO problem formulation. They also showed that the two bounds coincide with each other if the sources are independent and hence obtained an expression for the rate region [17]. The extremization functions that we are interested in are set-valued and the CEO only needs to know one value rather than the whole set of the function result. Under this setup, we give the fundamental limits of the minimum sum-rate to losslessly determine the extremizations. These results are in agreement with the results in [17] and [18] when the function is single-valued.

\section{B. Related Work-Lossy}

After Shannon introduced rate distortion function in source coding with a fidelity criterion [20], rate distortion theory was notably developed by Berger and Gallager [21], [22]. Recent work in rate distortion theory has been focused on lossy source coding by attempting to find efficient compression algorithms based on information theory. Rate distortion theory was extended to multi-terminal systems in the 1970's by Berger and Tung [23], [24]. For point-to-point rate distortion problems, Arimoto and Blahut proposed numerical calculation algorithms based on 
alternating optimization to find the channel capacity and rate distortion function [25], [26]. The convergence proof of Blahut and Arimoto' algorithms was developed by Csiszar [27] and Boukris [28]. The generic CEO problem from multi-terminal source coding theory was introduced by Berger [19] and the Quadratic Gaussian CEO rate region is known by Oohama [29] and Prabhakaran [30]. A general outer bound to the CEO problem, not necessarily required Quadratic Gaussian CEO problem, was derived by Wagner [31]. Lossy indirect function computation at the CEO was developed by Oohama [29] and Wyner [32]. An adaptation of the Blahut-Arimoto algorithm to the CEO model with independent sources is developed by the authors [33].

\section{Related Work-Scaler Quantization}

Recent work by Misra et al. considered the problem of distributed functional scalar quantization (DFSQ) [34]. By focusing on the high-rate regime and assuming a mean squared error (MSE) distortion, the authors are able to make several approximations to obtain distortion expressions that are optimal asymptotically (i.e., as the rate goes to infinity). We assume a different distortion measure, derive an exact expression for the distortion as a function of the quantizer parameters, and derive necessary conditions for optimal parameters. Moreover, our results hold for all rates.

Our focus on the use of SQs as an achievable scheme is motivated by several results concerning the optimality of a layered architecture of quantization followed by entropy coding. Zamir et al. considered the distributed encoding and centralized decoding of continuous valued sources and established that lattice quantization followed by SW encoding is optimal asymptotically in rate [35]. When the sources are Gaussian and the distortion is MSE, local vector quantizers followed by SW coding is optimal, not just asymptotically [36]. For discrete valued random variables, scalar quantization with block entropy encoding is optimal [37]. Each of the problem models considered in [35]-[37] can be understood as an instance of indirect distributed lossy source coding for the identity function.

\section{Related Work-Interaction}

Interactive communication is the scheme that allows message passing forward and backward multiple times between two or more terminals. For the two terminals' interactive communication problem of lossy source reproduction, Kaspi first characterized the rate region in [38]. Followed by this, Ishwar and Ma made some further contributions. They worked on both two and more than two terminals cases for computing any function of the sources in both lossy and lossless manner. They showed that interactive communication strictly improves the Wyner-Ziv rate-distortion function [39]. They also showed that in some distributed function computation problems, interactive communication can provide substantial benefits over non-interactive codes and infinite-many rounds of interaction may still improve the rate-region [14]. In Section VI] we consider resource allocation in the multiuser OFDMA systems that use rateless AWGN codes for downlink data transmission as a model of the distributed arg-max problem. We propose an interactive communication scheme for this resource allocation problem. This scheme is inspired by the ideas of selective multiuser diversity [40] (SMUD) scheme as well as the multi-predefined thresholds [41] 
scheme which is an extension of SMUD that set up multiple thresholds and allow the user nodes sending messages based on these thresholds.

\section{MODEL SPECIFICATION}

As stated previously, we are considering the $N$ user CEO problem for estimating either max, arg max, or the pair $(\max , \arg \max )$. The $i^{\text {th }}$ user observes the sequence $\boldsymbol{X}_{i} \triangleq\left(X_{i, s}: s \in[S]\right)$ of non-negative random variables

1 Let $\boldsymbol{X} \triangleq\left(\boldsymbol{X}_{i}: i \in[N]\right)$. We assume that the sources are independent and identically distributed (i.i.d.) across both users $(i)$ and the sequence $(s)$; that is

$$
f_{\boldsymbol{X}}(\boldsymbol{x})=\prod_{i=1}^{N} \prod_{s=1}^{S} f_{X}\left(x_{n, s}\right) .
$$

The quantities we are interested in for our problem are

$$
Z_{A}(s) \triangleq\left\{i \mid X_{i, s}=\max \left\{X_{i, s}: i \in[N]\right\}\right\}
$$

as the users with the maximum $s^{\text {th }}$ source output and

$$
Z_{M}(s) \triangleq \max \left\{X_{i, s}: i \in[N]\right\}
$$

as the maximum $s^{\text {th }}$ source output. Specifically for the arg max case, we consider a class of problems where we need not estimate the set $Z_{A}(s)$, but rather a representative user from this set.

\begin{tabular}{cl}
\hline Symbol & Meaning \\
\hline $\mathcal{C}(G(f))$ & set of all coloring method to color $G$ \\
$c\left(x_{i}\right)$ & coloring of source $x_{i}$ \\
$\ell$ & scalar quantizer decision boundaries \\
$\epsilon$ & fixed, but arbitrarily small value \\
$E_{k}$ & $\mathbb{E}\left[X \mid \ell_{k-1} \leq X \leq \ell_{k}\right]$ \\
$f$ & probability density function \\
$f_{k}$ & $f_{X}\left(\ell_{k}\right)$ \\
$F$ & cumulative density function \\
$F_{k}$ & $F_{X}\left(\ell_{k}\right)$ \\
$g$ & generic optimal resource allocation function \\
$G(V, E)$ & non directed graph with vertex set $\mathrm{V}$ and edge set $\mathrm{E}$ \\
$h$ & binary entropy function $-p \log _{2} p-(1-p) \log _{2}(1-p)$ \\
$H(\cdot)$ & Shannon entropy \\
$H_{G}$ & graph entropy \\
\hline
\end{tabular}

Continued on next page

${ }^{1}$ For any integer $n$, let $[n] \triangleq\{1, \ldots, n\}$ 
TABLE II-Continued from previous page

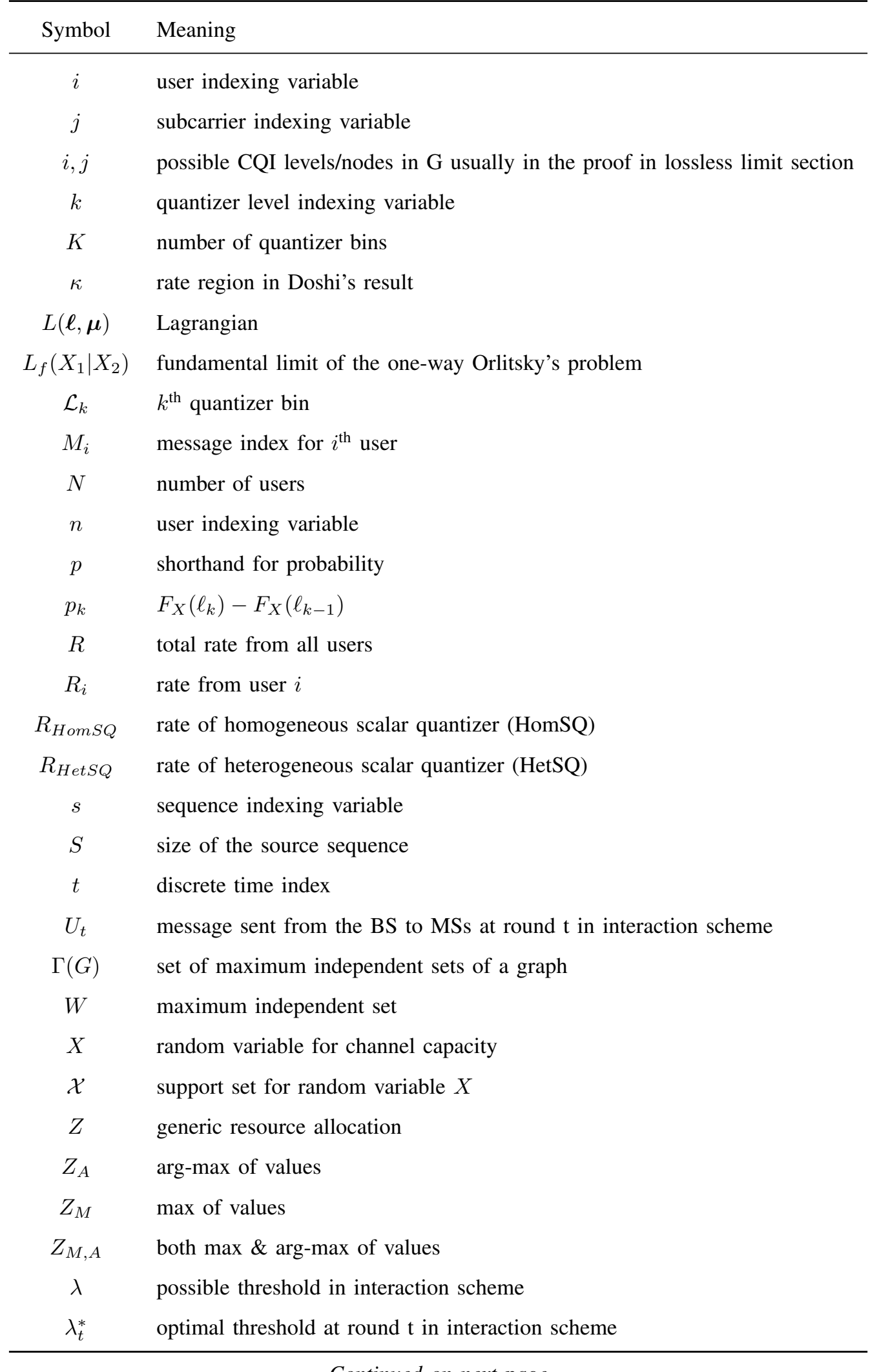

Continued on next page 
TABLE II-Continued from previous page

\begin{tabular}{cl}
\hline Symbol & Meaning \\
\hline$\mu$ & Lagrange multiplier (slope) for rate-distortion computation \\
$\nu$ & Lagrange multiplier associated with equality constraints \\
$\phi$ & encoding mapping \\
$\psi$ & decoding mapping
\end{tabular}

TABLE II: Notation Guide

\section{A. Distortion Measures}

Two typical distortion measures are Hamming and squared error, neither of which are appropriate for the problems of interest (Section [1. Examples 13). Hamming distortion is "all-or-nothing" in that all estimation errors are treated the same. Squared error distortion more heavily penalizes larger estimation errors, but treats under- and over-estimation the same. For the problems of interest, over-estimation needs to be more heavily penalized then under-estimation. With that in mind, we propose the following distortion measures. For estimating the max, the distortion measures linearly penalizes underestimation of the maximum value; we can think of this as the lost revenue (difference between what you could have gotten and what you got) when an asset is priced below market value. It also captures the loss when the estimated max rate exceeds the actual max; continuing the analogy, this is the case where an asset does not sell because it is priced above market value.

$$
d_{M}\left(\left(X_{1, s}, \ldots, X_{N, s}\right), \hat{Z}_{M}(s)\right)= \begin{cases}Z_{M}(s)-\hat{Z}_{M}(s) & \text { if } \hat{Z}_{M}(s) \leq Z_{M}(s) \\ Z_{M}(s) & \text { otherwise }\end{cases}
$$

For estimating the $\arg \max$, a similar distortion measure is utilized. The distortion measures the loss between source value of the user with the actual max and the source value for the user estimated to have the max. Unlike the previous case, the CEO cannot make an over-estimation error.

$$
d_{A}\left(\left(X_{1, s}, \ldots, X_{N, s}\right), \hat{Z}_{A}(s)\right)= \begin{cases}0 & \text { if } \hat{Z}_{A} \in Z_{A} \\ Z_{M}(s)-X_{\hat{Z}_{A}(s), s} & \text { otherwise }\end{cases}
$$

Finally, for estimating the pair of values (max, $\arg \max )$ we propose a distortion measure that is a hybrid of the previous two. The distortion is a combination of under-estimating the max value, provided the estimate does not exceed the value of the user estimated as having the max value. It also captures the loss due to over-estimation, both exceeding the estimated arg max user's value or exceeding the actual max value.

$$
d_{M, A}\left(\left(X_{1, s}, \ldots, X_{N, s}\right),\left(\hat{Z}_{M}(s), \hat{Z}_{A}(s)\right)\right)= \begin{cases}Z_{M}-\hat{Z}_{M}(s) & \text { if } \hat{Z}_{M}(s) \leq X_{\hat{Z}_{A}(s), s} \\ Z_{M} & \text { otherwise }\end{cases}
$$

Depending on the problem formulation being considered, let $d(s)$ be 
1) $d_{M, A}\left(\left(X_{1, s}, \ldots, X_{N, s}\right),\left(\hat{Z}_{M}(s), \hat{Z}_{A}(s)\right)\right)$;

2) $d_{A}\left(\left(X_{1, s}, \ldots, X_{N, s}\right), \hat{Z}_{A}(s)\right)$, or;

3) $d_{M}\left(\left(X_{1, s}, \ldots, X_{N, s}\right), \hat{Z}_{M}(s)\right)$

and define

$$
d\left(\left(\boldsymbol{X}_{1}, \ldots, \boldsymbol{X}_{N}\right), \hat{\boldsymbol{Z}}\right)=\frac{1}{S} \sum_{s=1}^{S} d(s)
$$

as the distortion between sequences. Finally, denote

$$
D=\mathbb{E}\left[d\left(\left(\boldsymbol{X}_{1}, \ldots, \boldsymbol{X}_{N}\right), \hat{\boldsymbol{Z}}\right)\right]
$$

where the expectation is with respect to joint distribution on the sources. In the next section, we consider the problem of finding the minimum sum rate necessary for computing the different extremization functions when the distortion is constrained $D=0$. Later, we will consider the problem of the minimum sum rate necessary for computing the different extremization functions with a non-zero upper bound on the distortion $D$.

\section{LOSSLESS EXTREMIZATION}

In this section, we determine the minimum amount of information necessary to remotely solve the extremization problems in a Shannon lossless sense. We begin by providing an achievable scheme for the arg max problem based on graph coloring in Section IV-A We then prove in Section IV-B that this scheme achieves a fundamental limit. We also show via a computation of the fundamental limits, that no rate can be saved relative to simply forwarding the observations in the max and (max, arg max) problems unless $\min \mathcal{X}=0$.

In [16], a related problem is considered in which the node observing $\boldsymbol{X}_{1}$ sends a message to the node observing $\boldsymbol{X}_{2}$ in such a manner that the function $\boldsymbol{f}^{S}\left(\boldsymbol{X}_{1}^{S}, \boldsymbol{X}_{2}^{S}\right)=\left[f\left(X_{1, s}, X_{2, s}\right) \mid s \in[S]\right]$, taking values from the set $\mathcal{Z}^{S}$, can be computed losslessly. In this problem, a rate $R$ is said to be achievable if for every $\epsilon>0$ there exists a sufficiently large $S$ and $K$ with $R \geq \frac{K}{S}$, and an encoder $\varphi: \mathcal{X}^{S} \rightarrow\{0,1\}^{K}$ and a decoder $\psi:\{0,1\}^{K} \times \mathcal{X}^{S} \rightarrow \mathcal{Z}^{S}$ such that $\mathbb{P}\left(\psi\left(\varphi\left(\boldsymbol{X}_{1}^{S}\right), \boldsymbol{X}_{2}^{S}\right) \neq \boldsymbol{f}^{S}\left(\boldsymbol{X}_{1}^{S}, \boldsymbol{X}_{2}^{S}\right)\right)<\epsilon$. Orlitsky and Roche proved that for given $X_{1}, X_{2}$ and $f$, the infimum of the set of achievable rates is

$$
L_{f}\left(X_{1} \mid X_{2}\right)=H_{G}\left(X_{1} \mid X_{2}\right)
$$

where $H_{G}\left(X_{1} \mid X_{2}\right)$ is the conditional graph entropy of the characteristic graph of this problem in [16]. The characteristic graph $G$ of $X_{1}, X_{2}$, and $f$ is a generalization of the definition given by Witsenhausen [15]. Its vertex set is the support set $\mathcal{X}$ of $X_{1}$, and distinct vertices $x_{1}, x_{1}^{\prime}$ are adjacent if there is a $x_{2}$ such that $p\left(x_{1}, x_{2}\right), p\left(x_{1}^{\prime}, x_{2}\right)>0$ and $f\left(x_{1}, x_{2}\right) \neq f\left(x_{1}^{\prime}, x_{2}\right)$. The conditional graph entropy is

$$
H_{G}\left(X_{1} \mid X_{2}\right) \triangleq \min _{W-X_{1}-X_{2}, X_{1} \in W \in \Gamma(G)} I\left(W ; X_{1} \mid X_{2}\right)
$$

where $\Gamma(G)$ is the set of all maximal independent sets in $G, W$ is a random variable that has $\Gamma(G)$ as its support set, and the minimization is over all conditional probabilities $p\left(w \mid x_{1}\right)$ which is supported on those maximal independent sets $w$ containing the vertex $x_{1}$, with the constraint that $W, X_{1}$ and $X_{2}$ form a Markov chain. 
Additionally, conditional graph entropy can be related to coloring a certain product graph. In particular, the OR-product graph $G_{1}^{S}\left(V_{S}, E_{S}\right)$, based on the characteristic graph $G_{1}$ of $X_{1}, X_{2}$ and $f$, has a vertex set $V_{S}=\mathcal{X}^{S}$, and distinct vertices $\left(x_{1,1}, \ldots, x_{1, S}\right),\left(x_{1,1}^{\prime}, \ldots, x_{1, S}^{\prime}\right)$ are connected if there exists an edge between $x_{1, s}$ and $x_{1, s}^{\prime}$ in $G_{1}$ for any $s$. In [42], Doshi et al. showed that minimum-entropy coloring the OR-product graph, followed by lossless compression of the colors with SW coding, yields a rate proportional to the conditional chromatic entropy, and can asymptotically reach the lower limit set out by the conditional graph entropy

$$
\lim _{S \rightarrow \infty} \min _{c \in \mathcal{C}_{\epsilon}\left(G_{1}^{S}(f)\right)} \frac{1}{S} H\left(c\left(X_{1}\right)\right)=H_{G}\left(X_{1} \mid X_{2}\right)
$$

where $\mathcal{C}_{\epsilon}\left(G_{1}^{S}(f)\right)$ is the set of all $\epsilon$-colorings of the product graph.

For the decentralized model where two users communicate with a CEO attempting to losslessly compute a function, Doshi et al. gave the rate region when the problem satisfies a given zig-zag condition, which requires that for any $\left(x_{1}, x_{2}\right)$ and $\left(x_{1}^{\prime}, x_{2}^{\prime}\right)$ in $\mathcal{X}_{1} \times \mathcal{X}_{2}, p\left(x_{1}, x_{2}\right)>0$ and $p\left(x_{1}^{\prime}, x_{2}^{\prime}\right)>0$ imply either $p\left(x_{1}, x_{2}^{\prime}\right)>0$ or $p\left(x_{1}^{\prime}, x_{2}\right)>0$ [18]. The key idea is to let each user do an $\epsilon$-coloring [18] of the OR-product graph of its own source and transmits the color by a SW code.

[18] showed in Theorem 16 that the rate-region for the aforementioned distributed function computation problem under the zig-zag condition is the set closure of $\kappa$, where $\kappa$ is the intersection of $\kappa^{\epsilon}$ for all $\epsilon>0$, and $\kappa^{\epsilon}$ is

$$
\kappa^{\epsilon}=\bigcup_{n=1}^{\infty} \bigcup_{\left(c_{x_{1}}^{n}, c_{x_{2}}^{n}\right)} \mathcal{R}^{n}\left(c_{x_{1}}^{n}, c_{x_{2}}^{n}\right)
$$

where the regions $\mathcal{R}^{n}\left(c_{x_{1}}^{n}, c_{x_{2}}^{n}\right)$ are given by

$$
\begin{aligned}
R_{x_{1}} & \geq \frac{1}{n} H\left(c_{x_{1}}^{n}\left(\mathbf{X}_{\mathbf{1}}\right) \mid c_{x_{2}}^{n}\left(\mathbf{X}_{\mathbf{2}}\right)\right) \\
R_{x_{2}} & \geq \frac{1}{n} H\left(c_{x_{2}}^{n}\left(\mathbf{X}_{\mathbf{2}}\right) \mid c_{x_{1}}^{n}\left(\mathbf{X}_{\mathbf{1}}\right)\right) \\
R_{x_{1}} & +R_{x_{2}} \geq \frac{1}{n} H\left(c_{x_{1}}^{n}\left(\mathbf{X}_{\mathbf{1}}\right), c_{x_{2}}^{n}\left(\mathbf{X}_{\mathbf{2}}\right)\right) .
\end{aligned}
$$

In Theorem 18, [18] showed that the difference of the minimum sum-rate and $H_{G}\left(X_{1} \mid X_{2}\right)+H_{G}\left(X_{2} \mid X_{1}\right)$ is bounded by

$$
H_{G}\left(X_{1} \mid X_{2}\right)+H_{G}\left(X_{2} \mid X_{1}\right)-\left(R_{x_{1}}+R_{x_{2}}\right) \leq \min \left\{I_{G_{1}}\left(X_{1} ; X_{2}\right), I_{G_{2}}\left(X_{1} ; X_{2}\right)\right\}
$$

where $I_{G_{1}}\left(X_{1} ; X_{2}\right)$ is the graph information of $X_{1}$, and the right hand side is zero when $X_{1}$ and $X_{2}$ are independent. Note that $H_{G}\left(X_{1} \mid X_{2}\right)=H_{G}\left(X_{1}\right)$ when the sources are independent, where the graph entropy $H_{G}\left(X_{1}\right)$ is

$$
H_{G}\left(X_{1}\right) \triangleq \min _{W-X_{1}-X_{2}, X_{1} \in W \in \Gamma(G)} I\left(W ; X_{1}\right) .
$$

Hence when the sources are independent, the rate-region is

$$
\begin{aligned}
& R_{x_{1}} \geq H_{G}\left(X_{1}\right) \\
& R_{x_{2}} \geq H_{G}\left(X_{2}\right) \\
& R_{x_{1}}+R_{x_{2}} \geq H_{G}\left(X_{1}\right)+H_{G}\left(X_{2}\right) .
\end{aligned}
$$


Doshi et al. consider a very general class of problems, for which in general it is necessary to express the rate region in terms of the $\epsilon$-coloring, which essentially is an valid coloring on a high probability subset of the characteristic graph. We will now show how to apply these ideas and related ones to the extremization problems under investigation. In particular, we will show in Section IV-B that we can achieve the fundamental limits of the sum-rate in the extremization problems by normally coloring the original characteristic graph as described in Section IV-A thereby removing the need for both OR-product graph and $\epsilon$-coloring.

\section{A. Achievable Schemes of Determining the arg max Function}

In this subsection we present an achievable scheme for determining the $\arg \max$ as we will show in Section IV-B. there is no need for sophisticated coding schemes for the max and both functions, as simple Huffman coding achieves the fundamental limits of the sum-rates for these functions.

We first consider $\mathrm{N}$ users, each observing $\boldsymbol{X}_{n}=\left(X_{n, s} \mid s \in\{1, \ldots, S\}, X_{n, s} \in \mathcal{X}\right)$ and assume that $\mathcal{X}=$ $\left\{\alpha_{1}, \alpha_{2}, \ldots, \alpha_{L}\right\}$ s.t. $0 \leq \alpha_{1}<\alpha_{2}<\ldots<\alpha_{L}$ and $\mathbb{P}\left(X=\alpha_{i}\right)>0$ for all $i \in[L]$ w.l.o.g.. For each element $X_{1, s}, \ldots, X_{N, s}$ of these sequences, we are interested in the aggregate rate required to enable the CEO to learn a $\hat{Z}_{A}(s)$ in the arg max such that

$$
\mathbb{E}\left[d_{A}\left(\left(X_{1, s}, \ldots, X_{N, s}\right), \hat{Z}_{A}(s)\right)\right]=0 .
$$

Definition 1. A rate $R$ will be said to be achievable if for every $\epsilon$ there exists $S, R_{1}, \ldots, R_{N}$ with $R=\sum_{n=1}^{N} R_{n}$, $\mathrm{N}$ encoder maps $\phi_{n}: \mathcal{X}^{S} \rightarrow\{0,1\}^{S \cdot R_{n}}, n \in\{1, \ldots, N\}$, and a decoder map $\psi:\{0,1\}^{S \cdot R_{1}} \times\{0,1\}^{S \cdot R_{2}} \ldots \times$ $\{0,1\}^{S \cdot R_{N}} \rightarrow\{1, \ldots, N\}^{S}$ such that $d_{A}\left(\left(X_{1, s}, \ldots, X_{N, s}\right), \psi\left(\phi_{1}\left(\boldsymbol{X}_{1}^{S}\right), \phi_{2}\left(\boldsymbol{X}_{2}^{S}\right), \ldots, \phi_{N}\left(\boldsymbol{X}_{N}^{S}\right)\right)\right)<\epsilon$.

We say a tie happens in the $\arg \max$ of the $s^{\text {th }}$ sources if two or more users attain the maximum value. Note that the arg max is not unique in such a case, because when a tie happens, the CEO can choose any user that achieves the maximum and will attain zero distortion. In other words, the extremization function is not uniquely determined everywhere. This will be useful when minimizing the amount of information necessary to determine this function.

Definition 2. A response from the $N$ users is $j$-ambigous if there are $j$ maximizers.

Lemma 1. The number of $j$-ambigous responses is

$$
A_{j, N}(K)=\left(\begin{array}{c}
N \\
j
\end{array}\right) \sum_{i=1}^{K-1} i^{N-j} .
$$

The number of possible deterministic tie-breaking arg max functions is

$$
C_{N}(K)=N \prod_{j=1}^{N} j^{A_{j, N}(K)}
$$

Proof: There are

$$
\left(\begin{array}{c}
N \\
j
\end{array}\right)(i-1)^{N-j}
$$

possible responses from the $N$ users such that $i$ is the index of the maximum value and $j$ is the number of maximizers. Summing over $i$ gives the total number of $j$-ambgious responses. For each of these responses, we have 


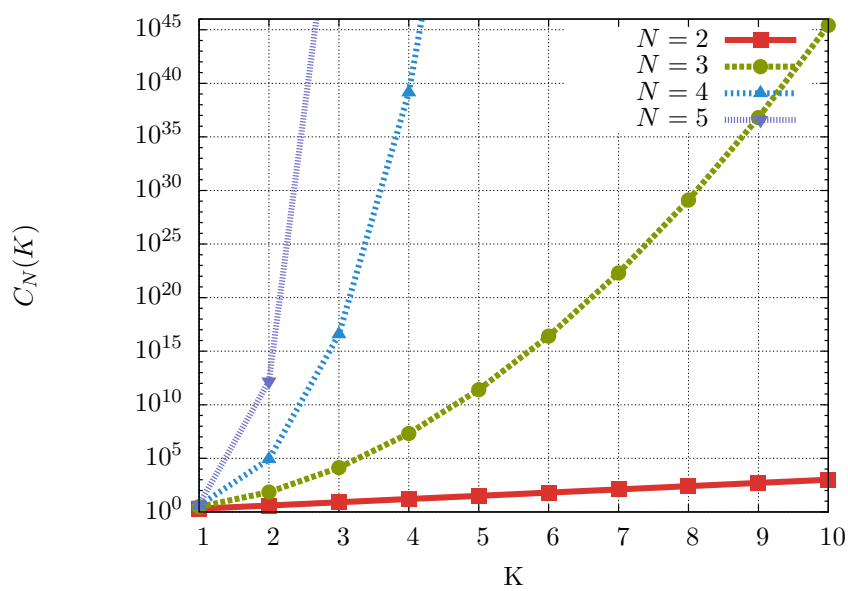

Fig. 3. The number of candidate arg max functions as a function of $K$ the number of values the users can take parameterized by $N$ the number of users.

$j$ possible values for the candidate $\arg \max$ function; if $i=1$, then we have $N$ possible values for the candidate function. Taking the product over $j$ and making suitable changes of variable gives the result of the lemma.

Remark. We have

$$
\begin{aligned}
& C_{2}(K)=2^{K} \\
& C_{3}(K)=3^{K} 2^{\frac{3 K(K-1)}{2}} \\
& C_{4}(K)=4^{K} 3^{2 K(K-1)} 2^{(K-1) K(2 K-1)} \\
& C_{5}(K)=5^{K} 4^{\frac{5 K(K-1)}{2}} 3^{\frac{10(K-1) K(2 K-1)}{6}} 2^{\frac{10 K^{2}(K-1)^{2}}{4}}
\end{aligned}
$$

These functions are plotted as a function of $K$ in Fig. 3 We see that the number of functions $\mathcal{X}^{N} \mapsto[N]$ that returns the unique maximizer when there is a single maximizer and deterministically breaks ties when there are more than one maximizer is extremely large, even for small values of $N$ and $K$.

We are able to realize a reduction in rate over the naïve approach of each user independently Huffman encoding their observations by searching over the space of functions that are consistent with arg max (defined formally 22) in Theorem 11. Despite the incredibly large search space, we develop a characterization of a subset of these candidate arg max functions and provide an expression for the rate acheived by these functions in Theorem 2 . In Section IV-B, we establish that this rate is in fact the best possible sum rate that can be attained.

Definition 3. A function $f: \mathcal{X}^{N} \rightarrow\{1, \ldots, N\}$ is a candidate $\arg \max$ function if and only if

$$
\mathbb{E}\left[d_{A}\left(\left(X_{1}, \ldots, X_{N}\right), f\left(X_{1}, \ldots, X_{N}\right)\right)\right]=0 .
$$

Let $\mathcal{F}_{A, N}$ be the set of all such candidate arg max functions with $N$ inputs. For any $f \in \mathcal{F}_{A, N}$, it indicates the index of a user attaining the max. 
Theorem 1. An achievable sum-rate for losslessly determining the argmax among a set of $N$ users is

$$
R_{A}=\min _{f_{N} \in \mathcal{F}_{A, N}} \sum_{n=1}^{N} \min _{c_{n} \in \mathcal{C}\left(G_{n}\left(f_{N}\right)\right)} H\left(c_{n}\left(X_{n}\right)\right)
$$

where the first minimization is over all candidate arg $\max$ functions, and $\mathcal{C}\left(G_{n}(f)\right)$ is the set of all colorings of the characteristic graph of user $n$ w.r.t. the function $f_{N}$.

Proof: This achievable scheme directly follows the result from [18] with a block size $S=1$ and by observing that an ordinary coloring is also an $\epsilon$-coloring. Following [18], we color the characteristic graph for each $\arg \max$ function and transmit the colors by a SW code. 23) is the minimum sum-rate over all such schemes w.r.t. all candidate arg max functions and all possible coloring schemes on the OR-product graph of size $S=1$.

In order to solve the optimizations in [23, the following two lemmas will be useful. Throughout the discussion below, we will use $\left\{\alpha_{i}, \alpha_{j}\right\} \in G$ to denote the existence of an edge between node $\alpha_{i}$ and $\alpha_{j}$ in the characteristic graph $G$, and use $\left\{\alpha_{i}, \alpha_{j}\right\} \notin G$ to denote that there is no such edge.

Lemma 2. For any function $f_{N} \in \mathcal{F}_{A, N}$ that determines the $\arg \max$, no 3 vertices can form an independent set in its characteristic graph $G_{i}\left(f_{N}\right)$ for any user $i$.

Proof: For any 3 vertices, there must exist two of them that their indices are not adjacent in number, say vertex $\alpha$ and vertex $\beta$, hence $\exists$ vertex $\gamma, \alpha<\gamma<\beta$ such that $f_{N}\left(x_{1}=\gamma, \ldots, x_{i-1}=\gamma, x_{i}=\alpha, x_{i+1}=\gamma, \ldots, x_{N}=\right.$ $\gamma) \neq f_{N}\left(x_{1}=\gamma, \ldots, x_{i-1}=\gamma, x_{i}=\beta, x_{i+1}=\gamma, \ldots, x_{N}=\gamma\right)$. Therefore, an edge must exist between $\alpha$ and $\beta$ in $G_{i}\left(f_{N}\right)$, and they can not be in the same independent set.

Lemma 3. For any function $f_{N} \in \mathcal{F}_{A, N}$ that determines the $\arg \max$, if $\{\alpha, \beta\} \notin G_{i}\left(f_{N}\right)$, then $\{\alpha, \beta\} \in$ $G_{n}\left(f_{N}\right) \forall n \in[N] \backslash\{i\}$.

Proof: Without loss of generality, we suppose $\alpha<\beta$. From the condition that $\{\alpha, \beta\} \notin G_{i}\left(f_{N}\right)$, we know that $\forall \boldsymbol{x}_{\backslash\{i\}} \in \mathcal{X}^{N-1}, f_{N}\left(x_{i}=\alpha, \boldsymbol{x}_{\backslash\{i\}}\right)=f_{N}\left(x_{i}=\beta, \boldsymbol{x}_{\backslash\{i\}}\right)$. In particular, we consider the following input sequences

$$
\begin{aligned}
& \boldsymbol{x}^{1}=\left(x_{1}^{1}, \ldots, x_{N}^{1}\right) \text { s.t. } x_{n}^{1}=\alpha \forall n \in[N], \\
& \boldsymbol{x}^{2}=\left(x_{1}^{2}, \ldots, x_{N}^{2}\right) \text { s.t. } x_{i}^{2}=\beta, x_{n}^{2}=\alpha \forall n \in[N] \backslash\{i\}, \text { and } \\
& \boldsymbol{x}^{3}=\left(x_{1}^{3}, \ldots, x_{N}^{3}\right) \text { s.t. } x_{j}^{3}=\beta, x_{n}^{3}=\alpha \forall n \in[N] \backslash\{j\} .
\end{aligned}
$$

Begin by observing that $f_{N}\left(\boldsymbol{x}^{2}\right)=i$ and $f_{N}\left(\boldsymbol{x}^{3}\right)=j$ since $\beta>\alpha$ and the positions associated with other users are all $\alpha$. Next, we observe that $f_{N}\left(\boldsymbol{x}^{1}\right)=f_{N}\left(\boldsymbol{x}^{2}\right)$ because $\{\alpha, \beta\} \notin G_{i}\left(f_{N}\right)$ by assumption. This then implies $f_{N}\left(\boldsymbol{x}^{1}\right) \neq f_{N}\left(\boldsymbol{x}^{3}\right)$, and hence there exists $\boldsymbol{x}_{\backslash\{j\}}=(\alpha, \ldots, \alpha)$ such that the function result differs for $x_{j}=\alpha$ and $x_{j}=\beta$, and there is an edge between $x_{j}=\alpha$ and $x_{j}=\beta$.

As we mentioned above, the minimum achievable sum-rate $R_{A}$ depends on how we break the ties (i.e. how we choose the candidate $\arg \max$ function). Denote $\mathcal{F}_{A, N}^{*}$ as the set of all candidate $\arg$ max functions that achieve $R_{A}$, the following theorem specifies the solution to the optimization problem introduced in Theorem 1 
Theorem 2. There exists a series of functions $\left\{f_{n}^{*} \mid n \in[N]\right\}$ where $f_{n}^{*}$ is a candidate $\arg \max$ function for $n$ users satisfying the properties that

1) $f_{1}^{*}(x)=1$ for any $x \in\left\{\alpha_{1}, \ldots, \alpha_{L}\right\}$,

2) $\forall \boldsymbol{x} \in \mathcal{S}_{n}^{-}\left(\alpha_{i}\right)$, where $\alpha_{i} \in \mathcal{X}$ and $\mathcal{S}_{n}^{-}\left(\alpha_{i}\right)=\left\{\left(x_{1}, \ldots, x_{n}\right) \mid x_{1}=\alpha_{i}, \max \left\{\boldsymbol{x}_{\backslash\{1\}}\right\}<\alpha_{i}\right\}$,

$$
f_{n}^{*}(\boldsymbol{x})=1,
$$

3) $\forall \boldsymbol{x} \in \mathcal{S}_{n}^{=}\left(\alpha_{i}\right)$, where $\alpha_{i} \in \mathcal{X}$ and $\mathcal{S}_{n}^{=}\left(\alpha_{i}\right)=\left\{\left(x_{1}, \ldots, x_{n}\right) \mid x_{1}=\alpha_{i}, \max \left\{\boldsymbol{x}_{\backslash\{1\}}\right\}=\alpha_{i}\right\}$,

$$
f_{n}^{*}(\boldsymbol{x})= \begin{cases}1 & \bmod (n, 2)=\bmod (i, 2) \\ f_{n-1}^{*}\left(\boldsymbol{x}_{\backslash\{1\}}\right)+1 & \text { otherwise }\end{cases}
$$

4) $\forall \boldsymbol{x} \in \mathcal{S}_{n}^{+}\left(\alpha_{i}\right)$, where $\alpha_{i} \in \mathcal{X}$ and $\mathcal{S}_{n}^{+}\left(\alpha_{i}\right)=\left\{\left(x_{1}, \ldots, x_{n}\right) \mid x_{1}=\alpha_{i}, \max \{\boldsymbol{x} \backslash\{1\}\}>\alpha_{i}\right\}$,

$$
f_{n}^{*}(\boldsymbol{x})=f_{n-1}^{*}\left(\boldsymbol{x}_{\backslash\{1\}}\right)+1
$$

such that

1) The minimum sum-rate achieved by graph coloring w.r.t. $f_{N}^{*}$ is

$$
R\left(f_{N}^{*}\right)=-(N-2) \sum_{i=1}^{L} p_{i} \log _{2} p_{i}-\sum_{i=1}^{L-1} p_{i, i+1} \log _{2} p_{i, i+1}-p_{1} \log _{2} p_{1}-p_{L} \log _{2} p_{L}
$$

where $p_{i}=\mathbb{P}\left(\mathbb{X}=\alpha_{i}\right)$ and $p_{i, i+1}=p_{i}+p_{i+1}$,

2) $f_{N}^{*} \in \mathcal{F}_{A, N}^{*}$, i.e. $R_{A}$ can be achieved by $f_{N}^{*}$.

Proof: See Appendix A1

Example 4 ( $N=3 L=4$ case). The properties that $f_{3}^{*}$ must obey become

1) $\forall \boldsymbol{x} \in \mathcal{S}_{3}^{-}\left(\alpha_{i}\right)$, where $\alpha_{i} \in \mathcal{X}$ and $\left.\mathcal{S}_{3}^{-}\left(\alpha_{i}\right)=\left\{\left(x_{1}, x_{2}, x_{3}\right) \mid x_{1}=\alpha_{i}, \max \left\{x_{2}, x_{3}\right\}\right\}<\alpha_{i}\right\}$,

$$
f_{3}^{*}(\boldsymbol{x})=1,
$$

2) $\forall \boldsymbol{x} \in \mathcal{S}_{3}=\left(\alpha_{i}\right)$, where $\alpha_{i} \in \mathcal{X}$ and $\mathcal{S}_{3}=\left(\alpha_{i}\right)=\left\{\left(x_{1}, x_{2}, x_{3}\right) \mid x_{1}=\alpha_{i}, \max \left\{x_{2}, x_{3}\right\}=\alpha_{i}\right\}$,

$$
f_{3}^{*}(\boldsymbol{x})= \begin{cases}1 & i \text { odd } \\ f_{2}^{*}\left(x_{2}, x_{3}\right)+1 & \text { otherwise }\end{cases}
$$

where

$$
f_{2}^{*}\left(x_{2}, x_{3}\right)= \begin{cases}1 & x_{2}=\alpha_{i}>x_{3} \\ 2 & x_{2}<x_{3}=\alpha_{i} \\ 1 & x_{2}=x_{3}=\alpha_{i}, i \text { even } \\ 2 & x_{2}=x_{3}=\alpha_{i}, i \text { odd }\end{cases}
$$

3) $\forall \boldsymbol{x} \in \mathcal{S}_{3}^{+}\left(\alpha_{i}\right)$, where $\alpha_{i} \in \mathcal{X}$ and $\mathcal{S}_{3}^{+}\left(\alpha_{i}\right)=\left\{\left(x_{1}, x_{2}, x_{3}\right) \mid x_{1}=\alpha_{i}, \max \left\{x_{2}, x_{3}\right\}>\alpha_{i}\right\}$,

$$
f_{3}^{*}(\boldsymbol{x})=f_{2}^{*}\left(x_{2}, x_{3}\right)+1
$$



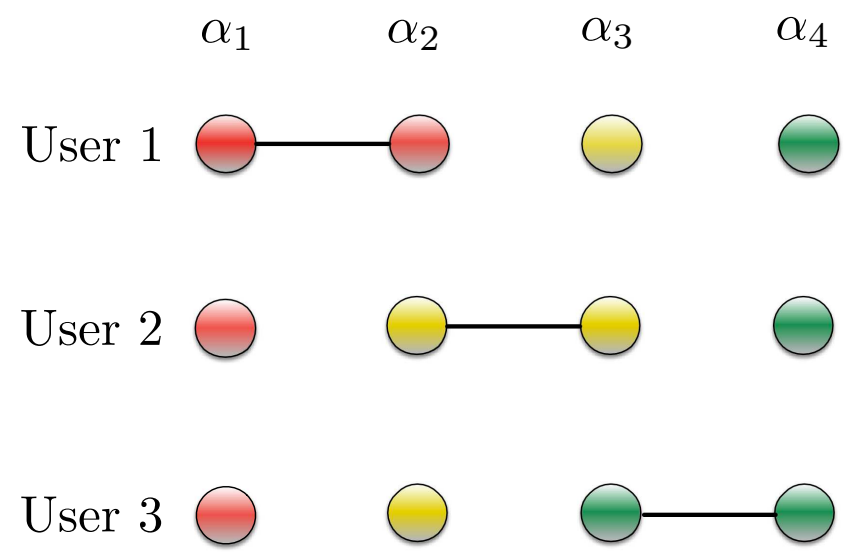

Fig. 4. Coloring the characteristic graph under the optimal function with $N=3$ and $L=4$

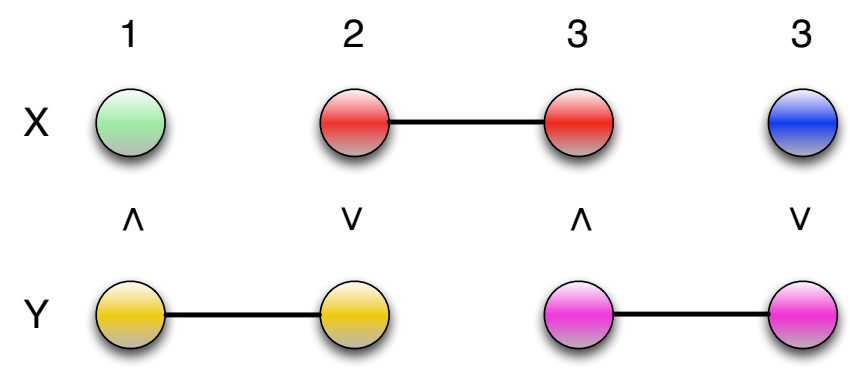

Fig. 5. Coloring the characteristic graph under the optimal function with $N=2$ and $L=4$

For convenience, we illustrate the complement characteristic graph as well as the coloring method in Fig. 4. Note that an edge connects two nodes in the complement graph represents that the two nodes forms an independent set in the original graph.

For the case that $N=2$, since there will be $L$ different ties that need to be distinguished, and $2^{L}$ different candidate functions that need to be considered, we have: 


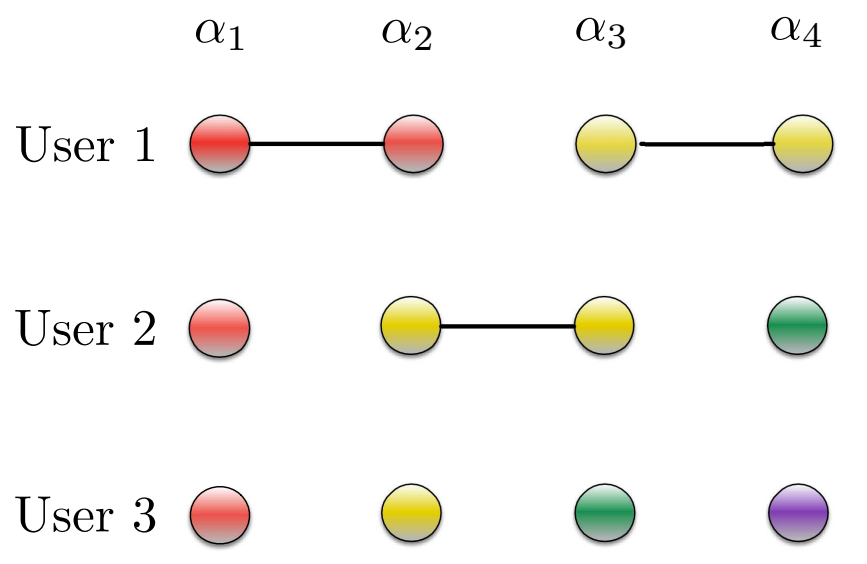

Fig. 6. Coloring the characteristic graph under the non-recursive optimal function with $N=3$ and $L=4$

Corollary 1 ( $N=2$ case). Among all $2^{L}$ arg max functions, the one that achieves the lowest sum-rate under minimum entropy graph coloring satisfies the property that for all $\alpha_{i} \in \mathcal{X}$,

$$
f_{2}^{*}\left(\alpha_{i}, \alpha_{i}\right)= \begin{cases}1, & i \text { odd } \\ 2, & i \text { even }\end{cases}
$$

Remark. Another type of candidate arg max function which leads to the complement characteristic graph as shown in Fig. 6 with rate

$$
R\left(f_{N}^{*}\right)=(N-2) H(X)+\min _{c_{1} \in \mathcal{C}\left(G_{1}\left(f_{2}^{*}\right)\right)} H\left(c_{1}\left(X_{1}\right)\right)+\min _{c_{2} \in \mathcal{C}\left(G_{2}\left(f_{2}^{*}\right)\right)} H\left(c_{2}\left(X_{2}\right)\right)
$$

although do not have the recursive property, can still achieve [28), and this structure can be interpreted as 2 of the $N$ users do graph coloring by Corollary 1 , and the rest of the $N-2$ users Huffman encode their own sources.

Having introduced this scheme, we will show in the next section that no scheme can have a higher rate-savings than this one. 


\section{B. Converse of Determining the Extremization Functions}

The following lemma is necessary to aid in drawing the conclusion that joint graph coloring achieves the fundamental limit and there is no benefit from the OR-product graph for arg max.

Lemma 4. For any given candidate $\arg \max$ function $f_{N} \in \mathcal{F}_{A, N}$, the conditional probability $p(w \mid x)$, which is supported on the maximal independent sets $w$ containing the vertices $x$, to achieve the minimum mutual information in the graph entropy expression (15) must be either 1 or 0 for all $n \in[N]$.

Proof: We prove this by showing that no vertex exists in two different maximal independent sets. Without loss of generality, we consider vertex $\alpha_{i}$ in $X_{1}$ 's characteristic graph. By Lemma 2 , the two maximal independent sets that $\alpha_{i}$ may belong to are $w_{1}=\left\{\alpha_{i-1}, \alpha_{i}\right\}$ and $w_{2}=\left\{\alpha_{i}, \alpha_{i+1}\right\}$. If vertex $\alpha_{i} \in w_{1}$ under the $\arg \max$ function $f_{N}$ (which means there is no edge between $\alpha_{i-1}$ and $\alpha_{i}$ ), then $\forall \boldsymbol{x}_{\backslash\{1\}} \in \mathcal{X}^{N-1}$, we have

$$
f_{N}\left(\alpha_{i}, \boldsymbol{x}_{\backslash\{1\}}\right)=f_{N}\left(\alpha_{i-1}, \boldsymbol{x}_{\backslash\{1\}}\right) .
$$

In particular, there exists $\boldsymbol{x}_{\backslash\{1\}}=\left(\alpha_{i}, \alpha_{i-1}, \ldots, \alpha_{i-1}\right)$ such that

$$
f_{N}\left(\alpha_{i}, \boldsymbol{x}_{\backslash\{1\}}\right)=f_{N}\left(\alpha_{i-1}, \boldsymbol{x}_{\backslash\{1\}}\right)=2,
$$

and obviously

$$
f_{N}\left(\alpha_{i+1}, \boldsymbol{x}_{\backslash\{1\}}\right)=1 .
$$

Therefore, $x_{1}=\alpha_{i}$ is connected to $x_{1}=\alpha_{i+1}$, and the set $w_{2}=\left\{\alpha_{i}, \alpha_{i+1}\right\}$ is not an independent set in $X_{1}$ 's characteristic graph, and we have

$$
p\left(w \mid x_{1}=\alpha_{i}\right)= \begin{cases}1, & w=\left\{\alpha_{i-1}, \alpha_{i}\right\} \\ 0, & \text { otherwise }\end{cases}
$$

Theorem 3. To losslessly determine the arg max, the fundamental limit of the sum-rate can be achieved by coloring the characteristic graph of each user, hence the OR-product graph is not necessary.

Proof: As reviewed at $(16)$, the fundamental limit of the sum-rate with independent sources problems is the sum of the graph entropy, i.e. $R_{A}^{*}=\sum_{n=1}^{N} R_{n}$ with $R_{n}=H_{G}\left(X_{n}\right)$. By Lemma 4 for any given candidate $\arg \max$ function,

$$
\begin{aligned}
H_{G}\left(X_{n}\right) & =\min _{p\left(w_{n} \mid x_{n}\right) \in\{0,1\}, w_{n} \in \Gamma\left(G_{n}\right)} I\left(W_{n} ; X_{n}\right) \\
& =\min _{p\left(w_{n} \mid x_{n}\right) \in\{0,1\}, w_{n} \in \Gamma\left(G_{n}\right)} H\left(W_{n}\right)-H\left(W_{n} \mid X_{n}\right) \\
& =\min _{p\left(w_{n} \mid x_{n}\right) \in\{0,1\}, w_{n} \in \Gamma\left(G_{n}\right)} H\left(W_{n}\right) .
\end{aligned}
$$

Note that the proof of Lemma 4 implies that the maximal independent sets are disjoint, and the fact that one can always Huffman encode the maximal independent sets with any given distribution. Consider using colors to 
represent the maximal independent sets, then Huffman encode the sets is the same as Huffman encode these colors. This color representation is a normal coloring method w.r.t. the characteristic graph since any two vertices connected by an edge will be in two different independent sets and no two maximal independent sets share the same color. Also note that when the maximal independents are disjoint, distinguish the vertices in the same independent set will result in a higher mutual information in the graph entropy optimization, since for any probabilities $p_{i}, p_{i+1}$ of the the nodes in a pairwise maximal independent set $\left\{\alpha_{i}, \alpha_{i+1}\right\}$, the difference of the mutual information will be

$$
\begin{aligned}
-p_{i} \log _{2} p_{i}-p_{i+1} \log _{2} p_{i+1}+\left(p_{i}+p_{i+1}\right) \log _{2}\left(p_{i}+p_{i+1}\right) & =-p_{i} \log _{2}\left(\frac{p_{i}}{p_{i}+p_{i+1}}\right)-p_{i+1} \log _{2}\left(\frac{p_{i+1}}{p_{i}+p_{i+1}}\right) \\
& =\left(p_{i}+p_{i+1}\right) h_{2}\left(\frac{p_{i}}{p_{i}+p_{i+1}}\right) \geq 0
\end{aligned}
$$

where $h_{2}()$ is the binary entropy function. Therefore 39 ) can be achieved by graph coloring. Since the scheme we present in Theorem 2 is the optimal coloring method w.r.t. the non-product characteristic graph, it must achieve the minimum in 39]. Therefore we have the following relationship for all $n \in[N]$ and the fundamental limit of the sum-rate can be achieved by Theorem 2

$$
H_{G}\left(X_{n}\right) \stackrel{(a)}{\leq} \lim _{S \rightarrow \infty} \frac{1}{S} \min _{c_{n} \in \mathcal{C}_{\epsilon}\left(G_{n}^{S}(f)\right)} H\left(c_{n}\left(X_{n}\right)\right) \stackrel{(b)}{\leq} \min _{c_{n} \in \mathcal{C}\left(G_{n}(f)\right)} H\left(c_{n}\left(X_{n}\right)\right) \stackrel{(c)}{=} H_{G}\left(X_{n}\right)
$$

where (a) holds by [42]; (b) holds by achievability: an ordinary coloring is also an $\epsilon$-coloring, and a valid ordinary coloring on the characteristic graph can be used in replication to achieve a valid coloring on the OR-product graph; and we have proved (c) above.

We now give the fundamental limit of the sum-rate in the problem that the CEO needs to determine $Z_{M}$ and $Z_{A, M}$ respectively.

Definition 4. A function $f: \mathcal{X}^{N} \rightarrow \mathcal{X}$ is a candidate max function if and only if

$$
\mathbb{E}\left[d_{M}\left(\left(X_{1}, \ldots, X_{N}\right), f\left(X_{1}, \ldots, X_{N}\right)\right)\right]=0 .
$$

Let $\mathcal{F}_{M, N}$ be the set of all such candidate max functions with $N$ inputs. For any $f \in \mathcal{F}_{M, N}$, it indicates the max.

Definition 5. A function $f: \mathcal{X}^{N} \rightarrow\{1, \ldots, N\} \times \mathcal{X}$ is a candidate (arg max, max) function if and only if

$$
\mathbb{E}\left[d_{A, M}\left(\left(X_{1}, \ldots, X_{N}\right), f\left(X_{1}, \ldots, X_{N}\right)\right)\right]=0 .
$$

Let $\mathcal{F}_{P, N}$ be the set of all such candidate (arg max, max) functions with $N$ inputs. For any $f \in \mathcal{F}_{P, N}$, it indicates both the index of a user attaining the max and the max.

Theorem 4. In the problem that the CEO needs to decide $\hat{Z}_{M}$, if $\min \mathcal{X}>0$, then the minimum sum-rate will be

$$
R_{M}^{*}=\sum_{n=1}^{N} H\left(X_{n}\right) .
$$


Proof: The distortion measure $d_{M}$ is

$$
d_{M}\left(\left(X_{1, s}, \ldots, X_{N, s}\right), \hat{Z}_{M}(s)\right)= \begin{cases}Z_{M}(s)-\hat{Z}_{M}(s) & \text { if } \hat{Z}_{M}(s) \leq Z_{M}(s) \\ Z_{M}(s) & \text { otherwise. }\end{cases}
$$

Given $\min \mathcal{X}>0, Z_{M}(s)$ can never be 0 , the only way to make 42 happen is to let $\hat{Z}_{M}(s)$ exactly estimate $Z_{M}(s)$, in other words, 42 is satisfied if and only if

$$
f\left(X_{1, s}, \ldots, X_{N, s}\right)=\max \left(X_{1, s}, \ldots, X_{N, s}\right)
$$

For any node pair $\left(\alpha_{i}, \alpha_{j}\right)$ in user $n$ 's characteristic graph, assume $i<j$ w.l.o.g., we will have $\left(\alpha_{i}, \alpha_{j}\right) \in G_{n}$ since there exists $\boldsymbol{x}_{\backslash\{n\}}=\left(\alpha_{i}, \ldots, \alpha_{i}\right)$ such that

$$
f\left(\alpha_{i}, \boldsymbol{x}_{\backslash\{n\}}\right) \neq f\left(\alpha_{j}, \boldsymbol{x}_{\backslash\{n\}}\right) .
$$

Therefore, the characteristic graph of user $n$ w.r.t. $f$ is complete, and $\Gamma\left(G_{n}\right)=\left\{\left\{\alpha_{i}\right\}: \alpha_{i} \in \mathcal{X}\right\}$, and the graph entropy is the same as the entropy of each source.

Remark. To achieve this limit, we simply need each user to Huffman encode its source.

Corollary 2. In the problem that the CEO needs to decide $\left(\hat{Z}_{A}, \hat{Z}_{M}\right)$, if $\min \mathcal{X}>0$, then the minimum sum-rate will be

$$
R_{A, M}^{*}=\sum_{n=1}^{N} H\left(X_{n}\right) .
$$

Proof: This directly follows the proof of Theorem 4, the characteristic graph is also complete if min $\mathcal{X}>0$.

Theorem 5. In the problem that the CEO needs to decide $\hat{Z}_{M}$, if $\min \mathcal{X}=0$, then the minimum sum-rate satisfies

$$
\begin{aligned}
R_{M}^{*} & =N H(X)+N\left(p_{1} \log _{2} p_{1}+p_{2} \log _{2} p_{2}-\left(p_{1}+p_{2}\right) \log _{2}\left(p_{1}+p_{2}\right)\right) \\
& =N H(X)-N\left(p_{1}+p_{2}\right) h_{2}\left(\frac{p_{1}}{p_{1}+p_{2}}\right)
\end{aligned}
$$

where $p_{1}=\mathbb{P}\left(X=\alpha_{1}=0\right)$ and $p_{2}=\mathbb{P}\left(X=\alpha_{2}\right)$.

Proof: Let $f \in \mathcal{F}_{M, N}$ satisfies that $f\left(\alpha_{1}, \ldots, \alpha_{1}\right)=\alpha_{2}$, then $\left(\alpha_{1}, \alpha_{2}\right) \notin G$ for the characteristic graph of each source $X_{i}$ w.r.t. $f$. The graph is not complete and the set of independent sets is $\Gamma\left(G_{n}\right)=\left\{\left\{\alpha_{1}, \alpha_{2}\right\},\left\{\alpha_{3}\right\}, \ldots,\left\{\alpha_{L}\right\}\right\}$ for all $n \in[N]$. Hence by a similar proof as in Theorem 2 and Theorem 3 , we have

$$
R_{M}^{*}=\sum_{n=1}^{N} H_{G}\left(X_{n}\right)
$$

with

$$
H_{G}\left(X_{n}\right)=-\left(p_{1}+p_{2}\right) \log _{2}\left(p_{1}+p_{2}\right)-\sum_{i=3}^{L} p_{i} \log _{2} p_{i}
$$

for all $n \in[N]$. 
Theorem 6. In the problem that the CEO needs to decide $\left(\hat{Z}_{A}, \hat{Z}_{M}\right)$, if $\min \mathcal{X}=0$, then the minimum sum-rate satisfies

$$
\begin{aligned}
R_{A, M}^{*} & =N H(X)+\left(p_{1} \log _{2} p_{1}+p_{2} \log _{2} p_{2}-\left(p_{1}+p_{2}\right) \log _{2}\left(p_{1}+p_{2}\right)\right) \\
& =N H(X)-\left(p_{1}+p_{2}\right) h_{2}\left(\frac{p_{1}}{p_{1}+p_{2}}\right)
\end{aligned}
$$

where $p_{1}=\mathbb{P}\left(X=\alpha_{1}\right)=\mathbb{P}(X=0)$ and $p_{2}=\mathbb{P}\left(X=\alpha_{2}\right)$.

Proof: Let $f=\left(f_{A}, f_{M}\right) \in \mathcal{F}_{P, N}$. Note that 43 is satisfied if and only if both 22) and (42) are satisfied, and hence $\left\{\alpha_{i}, \alpha_{j}\right\} \notin G_{n}$ in user $n$ 's characteristic graph if and only if $f_{A}\left(\alpha_{i}, \boldsymbol{x}_{\backslash\{n\}}\right)=f_{A}\left(\alpha_{j}, \boldsymbol{x}_{\backslash\{n\}}\right)$ and $f_{M}\left(\alpha_{i}, \boldsymbol{x}_{\backslash\{n\}}\right)=f_{M}\left(\alpha_{j}, \boldsymbol{x}_{\backslash\{n\}}\right)$ for all $\boldsymbol{x}_{\backslash\{n\}}=\left(x_{k} \mid k \in[N] \backslash\{n\}, x_{k} \in \mathcal{X}\right)$. Let $f_{A} \in \mathcal{F}_{A, N}$ be defined as in Theorem 2 and $f_{M} \in \mathcal{F}_{M, N}$ as in Theorem 5, then $\left\{\alpha_{1}, \alpha_{2}\right\} \notin G_{1}$, and all characteristic graphs other than $G_{1}$ are complete. The sets of independent sets are $\Gamma\left(G_{1}\right)=\left\{\left\{\alpha_{1}, \alpha_{2}\right\},\left\{\alpha_{3}\right\}, \ldots,\left\{\alpha_{L}\right\}\right\}$, and $\Gamma\left(G_{n}\right)=$ $\left\{\left\{\alpha_{1}\right\},\left\{\alpha_{2}\right\}, \ldots,\left\{\alpha_{L}\right\}\right\}$ for all $n \in[N] \backslash\{1\}$. By a similar proof as in Theorem 2 and Theorem 3 , we have

$$
R_{A, M}^{*}=\sum_{n=1}^{N} H_{G}\left(X_{n}\right)
$$

with

$$
H_{G}\left(X_{1}\right)=-\left(p_{1}+p_{2}\right) \log _{2}\left(p_{1}+p_{2}\right)-\sum_{i=3}^{L} p_{i} \log _{2} p_{i}
$$

and

$$
H_{G}\left(X_{n}\right)=H(X)
$$

for all $n \in[N] \backslash\{1\}$.

\section{Scaling in Number of Users}

In this subsection, we consider the rate saving performance of graph coloring in a large scale of $N$. Define the rate savings as the difference between the scheme that each user Huffman encode its source and the scheme by Theorem 2, i.e.

$$
\Delta_{A} \triangleq \sum_{n=1}^{N} H\left(X_{n}\right)-R_{A}^{*} .
$$

Theorem 7. To losslessly determine the $\arg \max$, the savings $\Delta_{A}$ is bounded by

$$
\max _{p_{1}, \ldots, p_{L}} \Delta_{A}=\max _{p_{1}, \ldots, p_{L}} \sum_{i=1}^{L-1}\left(p_{i}+p_{i+1}\right) h_{2}\left(\frac{p_{i}}{p_{i}+p_{i+1}}\right) \leq 2
$$

where $p_{i}=\mathbb{P}\left(X=\alpha_{i}\right)$, and hence the per user saving satisfies that

$$
\lim _{N \rightarrow \infty} \frac{\Delta_{A}}{N}=0 .
$$

Proof: See Appendix A2 
Corollary 3. In the problem that the CEO needs to decide the max, the per user saving $\Delta_{M} / N$ satisfies

$$
\lim _{N \rightarrow \infty} \frac{\Delta_{M}}{N}= \begin{cases}0 & \text { if } \min \mathcal{X}>0 \\ -\left(p_{1}+p_{2}\right) h_{2}\left(\frac{p_{1}}{p_{1}+p_{2}}\right) & \text { if } \min \mathcal{X}=0\end{cases}
$$

where $p_{1}=\mathbb{P}\left(x=\alpha_{1}\right)=\mathbb{P}(x=0)$ and $p_{2}=\mathbb{P}\left(x=\alpha_{2}\right)$.

Proof: See Appendix A3

Corollary 4. In the problem that the CEO needs to decide the pair (arg max, max), the per user saving $\Delta_{A, M} / N$ goes to 0 as $N$ goes to infinity.

Proof: Observe that

$$
R_{A}^{*} \leq R_{A, M}^{*} \leq \sum_{n=1}^{N} H\left(X_{n}\right)
$$

As we shall see in Section VI, the lack of savings in this lossless non-interactive problem structure stands in stark contrast to an interactive setup in which it can be shown that, by allowing the CEO to communicate with the users over multiple rounds, a substantial saving in sum rate relative to the the non-interactive scheme can be achieved [43] while still obtaining the answer losslessly. Additionally, as we will see in Section V] substantial rate savings can be obtained if we are willing to tolerate a small amount of loss.

\section{LOSSY EXTREMIZATION}

In the previous section, it was shown that having a CEO losslessly compute the max, arg max, or (max, arg max) of a set of distributed sources does not result in a significant rate savings relative to simply recovering all of the sources. For applications where reducing the rate is critical, tolerating bounded estimation error may be necessary. In this section, we consider the lossy variant of the function computation problem where the CEO need not determine the function output exactly. In particular, we first bound the best achievable rate-distortion tradeoff by computing the rate-distortion curves for each of the three functions with an adapted version of the Blahut-Arimoto algorithm in Section $\mathrm{V}$-A. Achievable schemes for each of the three functions based on scalar quantization followed by entropy encoding are then presented in Section $\mathrm{V}-\mathrm{B}$ and Section $\mathrm{V}-\mathrm{C}$ For certain problem instances, this scheme closely approximates the rate-distortion function as shown in Section V-D.

\section{A. Fundamental Limits-Multi-Source Blahut-Arimoto}

In this subsection, we first utilize a generalized Blahut-Arimoto (BA) algorithm to compute the sum-rate distortion function for the independent CEO extremization problems with discrete sources. We then show by Theorem 8 and Corollary 5 that the sum-rate distortion function for the case of continuous sources can be well approximated by discretizing the sources and applying the generalized BA algorithm from [33] to compute the rate distortion function for the discretized sources. In the limit as the discretization becomes arbitrarily fine, the discretized rate distortion 
function provides an $\epsilon$-lower bound for the continuous one. This calculated lower bound is used in Section V-D to measure the performance of the continuous quantizations we propose in Section $\mathrm{V}-\mathrm{B}$ and Section $\mathrm{V}-\mathrm{C}$

We first prove our discretization result for the classical single-source rate distortion function for continuous sources with bounded support.

Theorem 8. For any continuous source $X$ with bounded support set $\mathcal{X}=\left(x_{\min }, x_{\max }\right)$ and bounded PDF $f(x)$ if there exists a continuous quantizer

$$
\boldsymbol{Q}: \mathcal{X}^{S} \rightarrow\left\{1, \ldots, 2^{S R}\right\}
$$

a reconstruction function

$$
g:\left\{1, \ldots, 2^{S R}\right\} \rightarrow \hat{\mathcal{X}}=\left\{\hat{\boldsymbol{x}}_{1}, \ldots, \hat{\boldsymbol{x}}_{L}\right\}
$$

and a distortion metric

$$
d: \mathcal{X} \times \hat{\mathcal{X}} \rightarrow R^{+}
$$

that attain rate distortion pair $(R, D)$, where

$$
\begin{gathered}
L=2^{S R} \\
\hat{\boldsymbol{x}}_{\ell}=\left(\hat{x}_{\ell, 1}, \ldots, \hat{x}_{\ell, S}\right) \\
D=\mathbb{E}\left[\boldsymbol{d}\left(\boldsymbol{X}, \hat{\boldsymbol{X}}_{\ell}\right)\right] \\
\boldsymbol{d}\left(\boldsymbol{x}, \hat{\boldsymbol{x}}_{\ell}\right)=\frac{1}{S} \sum_{s=1}^{S} d\left(x_{s}, \hat{x}_{\ell, s}\right)
\end{gathered}
$$

with $d(\cdot)$ bounded above by $d_{m}$, and, when regarded as a function of $\boldsymbol{x}$ for a fixed $\hat{\boldsymbol{x}}_{\boldsymbol{\ell}}, \boldsymbol{d}\left(\boldsymbol{x}, \hat{\boldsymbol{x}}_{\ell}\right)$ has at most a finite number of discontinuous points, then there must exist a discrete source $Y_{K}$, a discrete quantizer

$$
\boldsymbol{Q}_{K}^{d}: \mathcal{Y}_{K}^{S} \rightarrow\left\{1, \ldots, 2^{S R}\right\}
$$

along with the same distortion metric $d$ and reconstruction mapping $g$ that attains rate $R_{K}=R$ and distortion $D_{K} \geq D$, where $Y_{K}=Q_{K}(X)$ is built by uniformly quantizing $X$ into $K$ intervals with the reconstruction levels $\mathcal{Y}_{K}=\left\{u_{k}: k \in[K]\right\}$. Further, $D_{K}$ can be arbitrarily close to $D$ for a large enough $K$, i.e.

$$
\lim _{K \rightarrow \infty} D_{K}=D
$$

Proof: Given a continuous source $X$ with $\mathcal{X}=\left[x_{\text {min }}, x_{\text {max }}\right]$ and PDF $f(x)$, the expected distortion is

$$
\begin{aligned}
D & =\mathbb{E}[\boldsymbol{d}(\boldsymbol{X}, \hat{\boldsymbol{X}})] \\
& =\int \boldsymbol{d}(\boldsymbol{x}, \boldsymbol{Q}(\boldsymbol{x})) f(\boldsymbol{x}) d \boldsymbol{x}
\end{aligned}
$$

where we denote $\hat{\boldsymbol{x}}_{\ell}=g(\boldsymbol{Q}(\boldsymbol{x}))$ by $\boldsymbol{Q}(\boldsymbol{x})$ for convenience, and

$$
\boldsymbol{d}(\boldsymbol{x}, \boldsymbol{Q}(\boldsymbol{x}))=\frac{1}{S} \sum_{s=1}^{S} d\left(x_{s},(\boldsymbol{Q}(\boldsymbol{x}))_{s}\right)
$$


Now let $Q_{K}$ that uniformly quantizes $X$ with $K$ intervals, i.e.

$$
Q_{K}: \mathcal{X} \rightarrow\left\{\mathcal{I}_{k}: k \in[K]\right\}
$$

where

$$
\mathcal{I}_{k}=\left(x_{\min }+\frac{k-1}{K}\left(x_{\max }-x_{\min }\right), x_{\min }+\frac{k}{K}\left(x_{\max }-x_{\text {min }}\right)\right) .
$$

Let $g_{K}$ that maps the intervals to the reconstruction levels, i.e.

$$
g_{K}:\left\{\mathcal{I}_{k}: k \in K\right\} \rightarrow\left\{u_{k}: k \in[K]\right\}
$$

with

$$
u_{k}=\underset{x \in \mathcal{I}_{k}}{\arg \min } d(x, Q(x)) .
$$

The discrete random variable $Y_{K}$ by discretizing $X$ is then defined on the support set

$$
\mathcal{Y}_{K}=\left\{u_{k}: k \in[K]\right\}
$$

with PMF

$$
\mathbb{P}\left[Y_{K}=u_{k}\right]=\int_{x \in \mathcal{I}_{k}} f(x) d x .
$$

Let $\boldsymbol{y}_{K}=\left(y_{K, s}: s \in[S]\right)$, the discrete quantizer $\boldsymbol{Q}_{K}^{d}$ satisfies

$$
\boldsymbol{Q}_{K}^{d}\left(\boldsymbol{y}_{K}\right)=\boldsymbol{Q}\left(\boldsymbol{y}_{K}\right)
$$

The distortion $D_{K}$ for quantization $Q_{K}^{d}$ will be

$$
D_{K}=\mathbb{E}\left[\boldsymbol{d}\left(\boldsymbol{Y}_{K}, \boldsymbol{Q}_{K}^{d}\left(\boldsymbol{Y}_{K}\right)\right]=\sum_{\boldsymbol{y}_{K} \in \mathcal{Y}_{K}^{S}} \boldsymbol{d}\left(\boldsymbol{y}_{K}, \boldsymbol{Q}_{K}^{d}\left(\boldsymbol{y}_{K}\right)\right) \mathbb{P}\left[\boldsymbol{Y}_{K}=\boldsymbol{y}_{K}\right]\right.
$$

where

$$
\boldsymbol{d}\left(\boldsymbol{y}_{K}, \boldsymbol{Q}_{K}^{d}\left(\boldsymbol{y}_{K}\right)\right)=\frac{1}{S} \sum_{s=1}^{S} d\left(y_{K, s},\left(\boldsymbol{Q}_{K}^{d}\left(\boldsymbol{y}_{K}\right)\right)_{s}\right) .
$$

Let $\boldsymbol{Q}_{K}(\cdot)$ quantize $\boldsymbol{x}$ element wise as $Q_{K}$ does. In addition, let

$$
\mathcal{I}\left(\boldsymbol{y}_{K}\right)=\left\{\boldsymbol{x} \in \mathcal{X}^{S} \mid \boldsymbol{Q}_{K}(\boldsymbol{x})=\boldsymbol{y}_{K}\right\}=\mathcal{I}_{1}\left(\boldsymbol{y}_{K}\right) \cup \mathcal{I}_{2}\left(\boldsymbol{y}_{K}\right) \cup \mathcal{I}_{3}\left(\boldsymbol{y}_{K}\right)
$$

be a subset of $\mathcal{X}^{S}$ that maps to $\boldsymbol{y}_{K}$ by $\boldsymbol{Q}_{K}(\cdot)$, where

$$
\begin{gathered}
\mathcal{I}_{1}\left(\boldsymbol{y}_{K}\right)= \begin{cases}\mathcal{I}\left(\boldsymbol{y}_{K}\right) & \text { if } \boldsymbol{Q}(\boldsymbol{x})=\boldsymbol{Q}\left(\boldsymbol{y}_{K}\right) \text { for all } \boldsymbol{x} \in \mathcal{I}\left(\boldsymbol{y}_{K}\right) \text { and } \boldsymbol{d}(\boldsymbol{x}, \hat{\boldsymbol{x}}) \text { is continuous on } \mathcal{I}\left(\boldsymbol{y}_{K}\right) \\
\emptyset & \text { otherwise }\end{cases} \\
\mathcal{I}_{2}\left(\boldsymbol{y}_{K}\right)= \begin{cases}\mathcal{I}\left(\boldsymbol{y}_{K}\right) & \text { if } \boldsymbol{Q}(\boldsymbol{x})=\boldsymbol{Q}\left(\boldsymbol{y}_{K}\right) \text { for all } \boldsymbol{x} \in \mathcal{I}\left(\boldsymbol{y}_{K}\right) \text { and } \boldsymbol{d}(\boldsymbol{x}, \hat{\boldsymbol{x}}) \text { is not continuous on } \mathcal{I}\left(\boldsymbol{y}_{K}\right) \\
\emptyset & \text { otherwise }\end{cases}
\end{gathered}
$$

and

$$
\mathcal{I}_{3}\left(\boldsymbol{y}_{K}\right)= \begin{cases}\mathcal{I}\left(\boldsymbol{y}_{K}\right) & \text { if } \exists \boldsymbol{x}_{1}, \boldsymbol{x}_{2} \in \mathcal{I}\left(\boldsymbol{y}_{K}\right) \text { such that } \boldsymbol{Q}\left(\boldsymbol{x}_{1}\right) \neq \boldsymbol{Q}\left(\boldsymbol{x}_{2}\right) \\ \emptyset & \text { otherwise }\end{cases}
$$


Clearly, $\mathcal{I}_{i}\left(\boldsymbol{y}_{K}\right) \cap \mathcal{I}_{j}\left(\boldsymbol{y}_{K}\right)=\emptyset$ for any $i \neq j, i, j \in\{1,2,3\}$. By comparing 70$\}$ and 79 , we have

$$
\begin{aligned}
D & =\int \boldsymbol{d}(\boldsymbol{x}, \boldsymbol{Q}(\boldsymbol{x})) f(\boldsymbol{x}) d \boldsymbol{x} \\
& =\sum_{\boldsymbol{y}_{K} \in \mathcal{Y}_{K}^{S}} \int_{\boldsymbol{x} \in \mathcal{I}\left(\boldsymbol{y}_{K}\right)} \boldsymbol{d}(\boldsymbol{x}, \boldsymbol{Q}(\boldsymbol{x})) f(\boldsymbol{x}) d \boldsymbol{x} \\
& =\sum_{\boldsymbol{y}_{K} \in \mathcal{Y}_{K}^{S}} \int_{\boldsymbol{x} \in \mathcal{I}\left(\boldsymbol{y}_{K}\right)}\left[\boldsymbol{d}(\boldsymbol{x}, \boldsymbol{Q}(\boldsymbol{x}))+\boldsymbol{d}\left(\boldsymbol{y}_{K}, \boldsymbol{Q}\left(\boldsymbol{y}_{K}\right)\right)-\boldsymbol{d}\left(\boldsymbol{y}_{K}, \boldsymbol{Q}\left(\boldsymbol{y}_{K}\right)\right)\right] f(\boldsymbol{x}) d \boldsymbol{x} \\
& =D_{K}+\sum_{\boldsymbol{y}_{K} \in \mathcal{Y}_{K}^{S}} \int_{\boldsymbol{x} \in \mathcal{I}\left(\boldsymbol{y}_{K}\right)}\left[\boldsymbol{d}(\boldsymbol{x}, \boldsymbol{Q}(\boldsymbol{x}))-\boldsymbol{d}\left(\boldsymbol{y}_{K}, \boldsymbol{Q}\left(\boldsymbol{y}_{K}\right)\right)\right] f(\boldsymbol{x}) d \boldsymbol{x}
\end{aligned}
$$

First observe by (71) and 75 that

$$
D=D_{K}+\sum_{\boldsymbol{y}_{K} \in \mathcal{Y}_{K}^{S}} \int_{\boldsymbol{x} \in \mathcal{I}\left(\boldsymbol{y}_{K}\right)}\left[\boldsymbol{d}(\boldsymbol{x}, \boldsymbol{Q}(\boldsymbol{x}))-\boldsymbol{d}\left(\boldsymbol{y}_{K}, \boldsymbol{Q}\left(\boldsymbol{y}_{K}\right)\right)\right] f(\boldsymbol{x}) d \boldsymbol{x} \geq D_{K}
$$

then we can further express 85 as

$$
\begin{aligned}
D & =D_{K}+\sum_{\boldsymbol{y}_{K} \in \mathcal{Y}_{K}^{S}} \int_{\boldsymbol{x} \in \mathcal{I}\left(\boldsymbol{y}_{K}\right)}\left[\boldsymbol{d}(\boldsymbol{x}, \boldsymbol{Q}(\boldsymbol{x}))-\boldsymbol{d}\left(\boldsymbol{y}_{K}, \boldsymbol{Q}\left(\boldsymbol{y}_{K}\right)\right)\right] f(\boldsymbol{x}) d \boldsymbol{x} \\
& =D_{K}+\sum_{\boldsymbol{y}_{K} \in \mathcal{Y}_{K}^{S}} \int_{\boldsymbol{x} \in \mathcal{I}_{1}\left(\boldsymbol{y}_{K}\right) \cup \mathcal{I}_{2}\left(\boldsymbol{y}_{K}\right) \cup \mathcal{I}_{3}\left(\boldsymbol{y}_{K}\right)}\left[\boldsymbol{d}(\boldsymbol{x}, \boldsymbol{Q}(\boldsymbol{x}))-\boldsymbol{d}\left(\boldsymbol{y}_{K}, \boldsymbol{Q}\left(\boldsymbol{y}_{K}\right)\right)\right] f(\boldsymbol{x}) d \boldsymbol{x} \\
& =D_{K}+A\left(\mathcal{I}_{1}\right)+A\left(\mathcal{I}_{2}\right)+A\left(\mathcal{I}_{3}\right)
\end{aligned}
$$

where

$$
A\left(\mathcal{I}_{i}\right)=\sum_{\boldsymbol{y}_{K} \in \mathcal{Y}_{K}^{S}} \int_{\boldsymbol{x} \in \mathcal{I}_{i}\left(\boldsymbol{y}_{K}\right)}\left[\boldsymbol{d}(\boldsymbol{x}, \boldsymbol{Q}(\boldsymbol{x}))-\boldsymbol{d}\left(\boldsymbol{y}_{K}, \boldsymbol{Q}\left(\boldsymbol{y}_{K}\right)\right)\right] f(\boldsymbol{x}) d \boldsymbol{x} .
$$

For any S-fold vector quantization $\boldsymbol{Q}(\cdot)$, we then have the following statements:

(a) $\forall \epsilon_{1}>0, \exists$ a large enough $K_{1}$ and a uniform quantization $Q_{K_{1}}$ such that $\forall \boldsymbol{x} \in \mathcal{I}_{1}\left(\boldsymbol{y}_{K_{1}}\right)$

$$
\boldsymbol{d}(\boldsymbol{x}, \boldsymbol{Q}(\boldsymbol{x}))-\boldsymbol{d}\left(\boldsymbol{y}_{K_{1}}, \boldsymbol{Q}\left(\boldsymbol{y}_{K_{1}}\right)\right) \leq \epsilon_{1}
$$

(b) $\forall \epsilon_{2}>0, \exists$ a large enough $K_{2}$ and a uniform quantization $Q_{K_{2}}$ such that

$$
\sum_{\boldsymbol{y}_{K_{2}} \in \mathcal{Y}_{K_{2}}^{S}} \mathbb{P}\left[\boldsymbol{X} \in \mathcal{I}_{2}\left(\boldsymbol{y}_{K_{2}}\right)\right]<\epsilon_{2} .
$$

(c) $\forall \epsilon_{3}>0, \exists$ a large enough $K_{3}$ and a uniform quantization $Q_{K_{3}}$ such that

$$
\sum_{\boldsymbol{y}_{K_{3}} \in \mathcal{Y}_{K_{3}}^{S}} \mathbb{P}\left[\boldsymbol{X} \in \mathcal{I}_{3}\left(\boldsymbol{y}_{K_{3}}\right)\right]<\epsilon_{3} .
$$

where 89 holds since $d(\cdot)$ is continuous on $\mathcal{I}_{1}\left(\boldsymbol{y}_{K}\right)$; 90 holds since $\boldsymbol{d}(\boldsymbol{x}, \hat{\boldsymbol{x}})$ has a finite number (i.e. $m$ ) of discontinuous points on $\mathcal{X}^{S} \times \hat{\mathcal{X}}$, and for given $\epsilon=\epsilon_{2} / m>0$, there must exist a large enough $K_{2}$ such that when $\mathcal{I}_{2}\left(\boldsymbol{y}_{K_{2}}\right) \neq \emptyset$,

$$
\mathbb{P}\left[\boldsymbol{X} \in \mathcal{I}_{2}\left(\boldsymbol{y}_{K_{2}}\right)\right]<\epsilon
$$


hence

$$
\begin{aligned}
\sum_{\boldsymbol{y}_{K_{2}} \in \mathcal{Y}_{K_{2}}^{S}} \mathbb{P}\left[\boldsymbol{X} \in \mathcal{I}_{2}\left(\boldsymbol{y}_{K_{2}}\right)\right] & =\sum_{\boldsymbol{y}_{K_{2}} \in \mathcal{Y}_{K_{2}}^{S}}\left(\mathbb{P}\left[\boldsymbol{X} \in \mathcal{I}_{2}\left(\boldsymbol{y}_{K_{2}}\right), \mathcal{I}_{2}\left(\boldsymbol{y}_{K_{2}}\right) \neq \emptyset\right]+\mathbb{P}\left[\boldsymbol{X} \in \mathcal{I}_{2}\left(\boldsymbol{y}_{K_{2}}\right), \mathcal{I}_{2}\left(\boldsymbol{y}_{K_{2}}\right)=\emptyset\right]\right) \\
& <m \epsilon+0 \\
& =\epsilon_{2} .
\end{aligned}
$$

Now we prove 91 also holds. Given a continuous quantizer

$$
\boldsymbol{Q}: \mathcal{X}^{S} \rightarrow\left\{1, \ldots, 2^{S R}\right\}
$$

a point $\boldsymbol{x} \in \mathcal{X}^{S}$ is a boundary point w.r.t. $\boldsymbol{Q}$ if for any $\epsilon>0$ there exist $\boldsymbol{x}_{1} \in \mathcal{X}^{S}, \boldsymbol{x}_{1} \neq \boldsymbol{x}$ such that

$$
\left\|\boldsymbol{x}_{1}-\boldsymbol{x}\right\|_{2}<\epsilon
$$

and

$$
\boldsymbol{Q}\left(\boldsymbol{x}_{1}\right) \neq \boldsymbol{Q}(\boldsymbol{x})
$$

Let $f(\boldsymbol{x})$ be a bounded PDF which is defined on $\mathcal{X}^{S} \subset \mathbb{R}^{S}$ with $\mathcal{X}=\left(x_{\min }, x_{\max }\right)$. For a continuous quantizer

$$
\boldsymbol{Q}: \mathcal{X}^{S} \rightarrow\left\{1, \ldots, 2^{S R}\right\}
$$

let $B(\boldsymbol{Q})$ be the set of all boundary points w.r.t. $\boldsymbol{Q}(\cdot)$. Since every k-dimensional subspace of $\mathbb{R}^{S}$ must have measure zero if $k<S$, by the definition of measure zero, we have that for any $\epsilon>0$, there exist open cubes $\mathcal{U}_{1}, \mathcal{U}_{2}, \ldots$ such that $B(\boldsymbol{Q}) \subseteq \cup_{i=1}^{\infty} \mathcal{U}_{i}$, and

$$
\sum_{i=1}^{\infty} \operatorname{vol}\left(\mathcal{U}_{i}\right)<\frac{\epsilon}{f_{\max }}
$$

where

$$
\begin{gathered}
\mathcal{U}_{i}=\left(a_{i, 1}, b_{i, 1}\right) \times\left(a_{i, 2}, b_{i, 2}\right) \times \cdots \times\left(a_{i, S}, b_{i, S}\right) \\
\operatorname{vol}\left(\mathcal{U}_{i}\right)=\left(b_{i, 1}-a_{i, 1}\right)\left(b_{i, 2}-a_{i, 2}\right) \cdots\left(b_{i, S}-a_{i, S}\right)
\end{gathered}
$$

and

$$
f_{\max }=\max _{\boldsymbol{x} \in \mathcal{X}^{S}} f(\boldsymbol{x})
$$

Hence

$$
\begin{aligned}
\mathbb{P}\left[\boldsymbol{X} \in \cup_{i=1}^{\infty} \mathcal{U}_{i}\right] & \leq \sum_{i=1}^{\infty} \int_{\boldsymbol{x} \in \mathcal{U}_{i}} f(\boldsymbol{x}) d \boldsymbol{x} \\
& \leq f_{\max }\left(\sum_{i=1}^{\infty} \int_{\boldsymbol{x} \in \mathcal{U}_{i}} d \boldsymbol{x}\right) \\
& =f_{\max } \sum_{i=1}^{\infty} \operatorname{vol}\left(\mathcal{U}_{i}\right) \\
& =\epsilon .
\end{aligned}
$$

In other words, the boundaries of the quantization levels have probability measure 0. 
Therefore, for any $\epsilon>0$ and any $S$, there exists $K \geq \max \left\{K_{1}, K_{2}, K_{3}\right\}$ such that

$$
\begin{aligned}
D & =D_{K}+A\left(\mathcal{I}_{1}\right)+A\left(\mathcal{I}_{2}\right)+A\left(\mathcal{I}_{3}\right) \\
& \leq D_{K}+\sum_{\boldsymbol{y}_{K} \in \mathcal{Y}_{K}} \int_{\boldsymbol{x} \in \mathcal{I}_{1}\left(\boldsymbol{y}_{K}\right)} \epsilon_{1} f(\boldsymbol{x}) d \boldsymbol{x}+\sum_{\boldsymbol{y}_{K} \in \mathcal{Y}_{K}} \int_{\boldsymbol{x} \in \mathcal{I}_{2}\left(\boldsymbol{y}_{K}\right)} d_{m} f(\boldsymbol{x}) d \boldsymbol{x}+\sum_{\boldsymbol{y}_{K} \in \mathcal{Y}_{K}} \int_{\boldsymbol{x} \in \mathcal{I}_{3}\left(\boldsymbol{y}_{K}\right)} d_{m} f(\boldsymbol{x}) d \boldsymbol{x} \\
& =D_{K}+\epsilon_{1} \sum_{\boldsymbol{y}_{K} \in \mathcal{Y}_{K}} \mathbb{P}\left[\boldsymbol{X} \in \mathcal{I}_{1}\left(\boldsymbol{Y}_{K}\right)\right]+d_{m} \sum_{\boldsymbol{y}_{K} \in \mathcal{Y}_{K}} \mathbb{P}\left[\boldsymbol{X} \in \mathcal{I}_{2}\left(\boldsymbol{Y}_{K}\right)\right]+d_{m} \sum_{\boldsymbol{y}_{K} \in \mathcal{Y}_{K}} \mathbb{P}\left[\boldsymbol{X} \in \mathcal{I}_{3}\left(\boldsymbol{Y}_{K}\right)\right] \\
& \leq D_{K}+\epsilon_{1}+\epsilon_{2} d_{m}+\epsilon_{3} d_{m}
\end{aligned}
$$

and

$$
\lim _{K \rightarrow \infty} D_{K}=D
$$

Corollary 5. In the CEO problem for estimating a function $f(\cdot)$ of $N$ independent observations, user $n$ observe $\boldsymbol{X}_{n}=\left(X_{n, s}: s \in[S]\right)$, where $X_{n, s}$ is a continuous random variable drawn from a bounded support set $\mathcal{X}=$ $\left(x_{\text {min }}^{n}, x_{\text {max }}^{n}\right)$ with a bounded PDF $f_{n}(x)$. If for each user there exists a continuous quantizer $\boldsymbol{Q}_{n}$

$$
\boldsymbol{Q}_{n}: \mathcal{X}_{n}^{S} \rightarrow\left\{1, \ldots, 2^{S R_{n}}\right\}
$$

a joint reconstruction function

$$
g:\left\{1, \ldots, 2^{S R_{1}}\right\} \times\left\{1, \ldots, 2^{S R_{2}}\right\} \times \cdots \times\left\{1, \ldots, 2^{S R_{N}}\right\} \rightarrow \hat{\mathcal{X}}=\left\{\hat{\boldsymbol{x}}_{1}, \ldots, \hat{\boldsymbol{x}}_{L}\right\}
$$

and a distortion metric

$$
d: \mathcal{X}_{1} \times \cdots \times \mathcal{X}_{N} \times \hat{\mathcal{X}} \rightarrow R^{+}
$$

that attain rate distortion pair $(\boldsymbol{R}, D)$, where

$$
\begin{gathered}
\boldsymbol{R}=\left(R_{1}, \ldots, R_{N}\right) \\
\hat{\boldsymbol{x}}_{\ell}=\left(\hat{x}_{\ell, 1}, \ldots, \hat{x}_{\ell, S}\right) \\
D=\mathbb{E}\left[\boldsymbol{d}\left(\boldsymbol{X}_{1}, \ldots, \boldsymbol{X}_{N}, \hat{\boldsymbol{X}}\right)\right] \\
\boldsymbol{d}\left(\boldsymbol{x}_{1}, \ldots, \boldsymbol{x}_{N}, \hat{\boldsymbol{x}}_{\ell}\right)=\frac{1}{S} \sum_{s=1}^{S} d\left(x_{1, s}, \ldots, x_{N, s}, \hat{x}_{\ell, s}\right)
\end{gathered}
$$

with $d(\cdot)$ bounded above by $d_{m}$, and, when regarded as a function of $\boldsymbol{x}_{1}, \ldots, \boldsymbol{x}_{N}$ for a fixed $\hat{\boldsymbol{x}}_{\ell}, \boldsymbol{d}\left(\boldsymbol{x}_{1}, \ldots, \boldsymbol{x}_{N}, \hat{\boldsymbol{x}}_{\ell}\right)$ has at most a finite number of discontinuous points, then there must exist discrete sources $\left\{Y_{K_{n}}: n \in[N]\right\}$, a series of discrete quantizers

$$
\boldsymbol{Q}_{n}^{d}: \mathcal{Y}_{K_{n}}^{S} \rightarrow\left\{1, \ldots, 2^{S R_{n}}\right\}
$$

along with the same distortion metric $d(\cdot)$ and reconstruction mapping $g$ that attains rate region $\boldsymbol{R}_{K}=\boldsymbol{R}_{\text {and }}$ average distortion $D_{K} \geq D$, where $Y_{K_{n}}=Q_{K_{n}}\left(X_{n}\right)$ is built by uniformly quantizing $X_{n}$ into $K_{n}$ intervals with reconstruction levels $\left\{u_{k, n}: k \in\left[K_{n}\right]\right\}$. Further, $D_{K}$ can be arbitrarily close to $D$ for a large enough $K$, i.e.

$$
\lim _{K \rightarrow \infty} D_{K}=D
$$


Proof: The proof follows along the same lines as the Theorem 8

It follows directly from Theorem 8 and Corollary 5 that a tight lower bound for the continuous source distortion rate functions for the extremization problems of interest can be computed via the algorithm presented in [33] applied to a suitably fine discretization of the continuous source.

In Fig. 7, we show example rate distortion functions for the three extremization problems for a fixed number of users $N=2$ with Uniform $(0,1)$ sources; we also show how the rate distortion function scales with the number of users $N \in\{2,3,4\}$ for the case of the arg max function. Observer in the first three plots, that difference between the approximations of the continuous source rate distortion functions is rapidly diminishing with the discretization parameters $K$. Looking at the fourth plot, it appears that the rate distortion function scales neglibly in the number of users. In fact, based on the performance of the SQs discussed in Section V-D (Fig. 13), we observe that the rate distortion function must decrease as the number of users $N$ grows large. Note that for our plots of rate versus distortion, we normalize the distortion by the expected value of the maximum, i.e.,

$$
\frac{\mathbb{E}\left[d\left(\left(X_{1}, \ldots, X_{N}\right), \hat{z}\right)\right]}{\mathbb{E}\left[\max \left\{X_{1}, \ldots, X_{N}\right\}\right]} .
$$

In the rest of Section $\mathrm{V}$, we will discuss quantization designs for the independent CEO extremization problems with continuous source random variables. The lower bounds that we compute based on Theorem 8 and Corollary 5 (and shown in Fig. 7) will be used as the fundamental limits to measure the performance of the quantization schemes we propose in Section $\mathrm{V}-\mathrm{B}$ and Section $\mathrm{V}-\mathrm{C}$

\section{B. Scalar Quantizers for arg max}

In this section, we consider the design of SQs as an achievable scheme and compare their performance to computed rate-distortion functions. We first consider the case where all users are using the same quantizer and derive an expression for the resulting distortion. Using this expression, we pose two non-linear optimization problems: first, minimize distortion for a given number of bins, and; second, minimize distortion for a given number of bins subject to a constraint on the entropy of the quantizer output. We provide first order necessary conditions for the optimal quantizer for both non-linear optimizations. We then argue that the same distortion performance can be achieved with a smaller sum rate by utilizing different quantizers at each user. We show that the design of the HetSQ can be accomplished via the same design procedure as for the HomSQ.

Let $X_{i}: i=1, \ldots, N$ be the sources for the $N$ users and let $Z_{A}$ be the index of the user with maximum value. Unlike previous sections, we assume continous (instead of discrete) random variables $X_{i}: i=1, \ldots, N$. As before, we still assume they are i.i.d. with common PDF $f(x), \operatorname{CDF} F(x)$, and support set $\mathcal{X} \subseteq \mathbb{R}_{+}$.

1) Homogeneous Scalar Quantizers: Normally, a SQ is specified as a set of decision boundaries and reconstruction levels [44]. For the estimating the arg max, we do not need the CEO to produce estimates for $X_{i}: i=1, \ldots, N$ or even $X_{Z_{A}}$ (i.e., the value of the maximum source). We can therefore specify the quantizer with just a set of decision boundaries $\left\{\ell_{k}: k=0, \ldots, K\right\}$ which divide the support set $\mathcal{X}$ into $K$ intervals

$$
\mathcal{L}_{k}=\left[\ell_{k-1}, \ell_{k}\right] \quad k=1, \ldots, K
$$




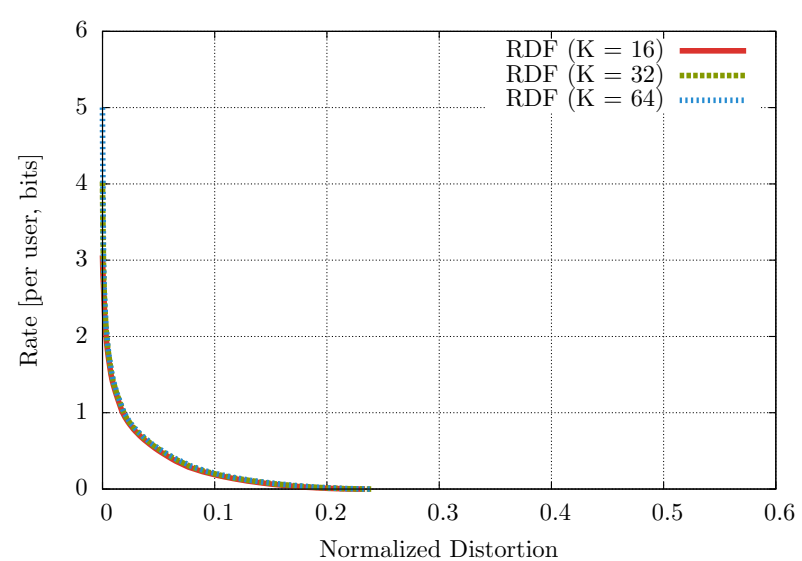

(a)

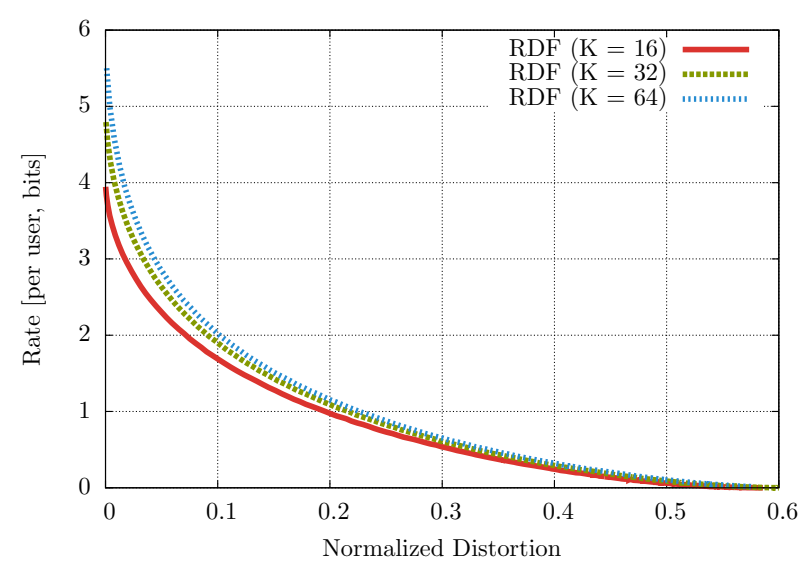

(c)

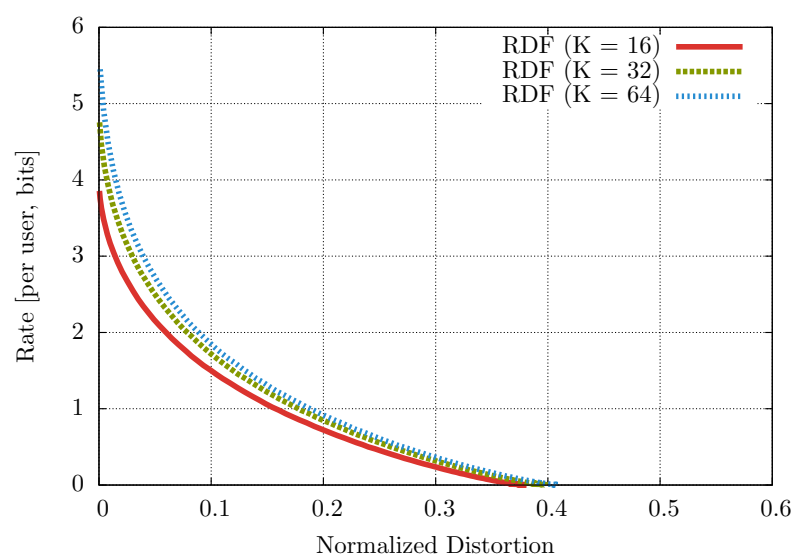

(b)

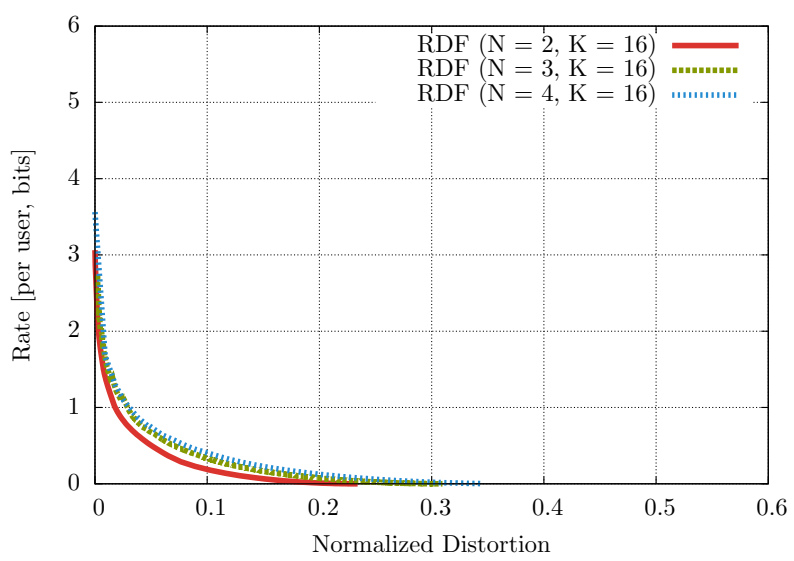

(d)

Fig. 7. (a)-(c): Rate distortion function for $N=2$ uniformly distributed sources as a function of the discretization parameter $K$; (d): Rate distortion function for $\arg \max$ as a function of $N$ with a fixed $K=16$

where $\ell_{0} \triangleq \inf \mathcal{X}$ and $\ell_{K} \triangleq \sup \mathcal{X}$. Let $U_{i} \in\{1, \ldots, K\}$ indicate the interval in which user $i$ 's observed value lies. The CEO will pick user $i$ if $U_{i}>U_{i^{\prime}}$ for all $i^{\prime} \neq i$ and will randomly pick a user from $\arg \max U_{i}$ otherwise; we denote the estimate so obtained as $X_{\hat{Z}_{A}}$.

For notational brevity, we define the following: $E_{j} \triangleq \mathbb{E}\left[X \mid \ell_{j-1} \leq X \leq \ell_{j}\right], f_{j}=f\left(\ell_{j}\right), F_{j} \triangleq F\left(\ell_{j}\right)$, and $p_{j} \triangleq \mathbb{P}\left(\ell_{j-1} \leq X \leq \ell_{j}\right)$

Lemma 5. Let $\left(X_{i}: i \in[N]\right)$ be a collection of i.i.d. random variables with cdf $F(x)$ and pdf $f(x)$ and

$$
Z_{A} \triangleq\left\{i \mid X_{i}=\max \left\{X_{1}, \ldots, X_{N}\right\}, i \in[N]\right\}
$$

The expected value of the max is

$$
\mathbb{E}\left[X_{i} \mid i \in Z_{A}\right]=\int_{\inf \mathcal{X}}^{\sup \mathcal{X}} x N F^{N-1}(x) f(x) \mathrm{d} x .
$$


Proof: Omitted for brevity.

Theorem 9. Let $\left(X_{i}: i \in[N]\right)$ be a collection of i.i.d. random variables with $c d f F(x)$ and $p d f f(x)$ and

$$
Z_{A} \triangleq\left\{i \mid X_{i}=\max \left\{X_{1}, \ldots, X_{N}\right\}, i \in[N]\right\}
$$

The expected value of the estimated arg max when using HomSQs with $K$ intervals is

$$
\mathbb{E}\left[X_{\hat{Z}_{A}}\right]=\sum_{j=1}^{K}\left[E_{j}\left(F_{j}^{N}-F_{j-1}^{N}\right)\right] .
$$

Proof: See Appendix A4

Recall that for a collection of i.i.d. random variables $X_{i}: i \in[N]$ ), the CDF of maximum $Z=\max _{i} X_{i}$ is given as

$$
F_{Z}(z)=F_{X}^{N}(z)
$$

We see then that an alternative and more intuitive way to view 119 is given as

$$
\mathbb{E}\left[X_{\hat{Z}_{A}}\right]=\sum_{j=1}^{K} E_{j} \mathbb{P}\left(\ell_{j-1} \leq X_{Z_{A}} \leq \ell_{j}\right) \text {. }
$$

Lemma 6.

$$
\begin{aligned}
\frac{\partial E_{k}}{\partial \ell_{k-1}} & =f_{k-1} \frac{E_{k}-\ell_{k-1}}{p_{k}} \\
\frac{\partial E_{k}}{\partial \ell_{k}} & =f_{k} \frac{\ell_{k}-E_{k}}{p_{k}}
\end{aligned}
$$

Proof: Follows from application of the quotient rule and Leibniz's rule.

\section{Lemma 7.}

$$
\frac{\partial \mathbb{E}\left[X_{\hat{Z}_{A}}\right]}{\partial \ell_{k}}=f_{k}\left[\frac{\left(F_{k+1}^{N}-F_{k}^{N}\right)\left(E_{k+1}-\ell_{k}\right)}{p_{k+1}}+\frac{\left(F_{k}^{N}-F_{k-1}^{N}\right)\left(\ell_{k}-E_{k}\right)}{p_{k}}-N F_{k}^{N-1}\left(E_{k+1}-E_{k}\right)\right]
$$

Proof: See Appendix A5

Corollary 6. For $N=2$, the above simplifies to

$$
\frac{\partial \mathbb{E}\left[X_{\hat{Z}_{A}}\right]}{\partial \ell_{k}}=f_{k}\left[\int_{\ell_{k-1}}^{\ell_{k+1}}\left(x-\ell_{k}\right) f(x) \mathrm{d} x\right]
$$

Proof: See Appendix A6

a) Minimum Distortion: For a given number of intervals $K$, the decision boundaries $\left\{\ell_{k}: k=0, \ldots, K\right\}$ that minimize the expected distortion are given by the solution to the following non-linear optimization:

$$
\begin{array}{ll}
\underset{\ell}{\operatorname{minimize}} & D(\ell) \\
\text { subject to } & \ell_{k-1} \leq \ell_{k} \quad k=1, \ldots, K .
\end{array}
$$


Theorem 10. If $\left\{\ell_{k}^{*}: k=0, \ldots, K\right\}$ is an optimal solution to 125 then there exists $\mu_{K}^{*} \geq 0$ for $k=1, \ldots, K$ such that

$$
\begin{gathered}
f_{k}\left[\frac{\left(F_{k+1}^{N}-F_{k}^{N}\right)\left(\ell_{k}^{*}-E_{k+1}\right)}{p_{k+1}}+\frac{\left(F_{k}^{N}-F_{k-1}^{N}\right)\left(E_{k}-\ell_{k}^{*}\right)}{p_{k}}-N F_{k}^{N-1}\left(E_{k}-E_{k+1}\right)\right]-\mu_{k}^{*}+\mu_{k+1}^{*}=0 \\
\mu_{k}^{*}\left(\ell_{k-1}^{*}-\ell_{k}^{*}\right)=0 .
\end{gathered}
$$

Proof: The Lagrangian associated with this problem is

$$
L(\ell, \boldsymbol{\mu})=D(\ell)+\sum_{k=1}^{K} \mu_{k}\left(\ell_{k-1}-\ell_{k}\right)
$$

Taking the derivative w.r.t. $\ell_{i}$ gives

$$
\frac{\partial L(\ell, \boldsymbol{\mu})}{\partial \ell_{k}}=\frac{\partial D(\ell)}{\partial \ell_{k}}-\mu_{k}+\mu_{k+1}
$$

where

$$
\frac{\partial D(\ell)}{\partial \ell_{k}}=-\frac{\partial \mathbb{E}\left[X_{\hat{Z}_{A}}\right]}{\partial \ell_{k}} .
$$

The result follows from setting the above equal to zero and complementary slackness.

Corollary 7. For $N=2$, the above simplifies to

$$
\begin{aligned}
f_{k}\left[\int_{\ell_{k-1}^{*}}^{\ell_{k+1}^{*}}\left(\ell_{k}^{*}-x\right) f(x) \mathrm{d} x\right]-\mu_{k}^{*}+\mu_{k+1}^{*}=0 \\
\mu_{k}^{*}\left(\ell_{k-1}^{*}-\ell_{k}^{*}\right)=0
\end{aligned}
$$

Remark. In Section $\mathrm{V}-\mathrm{D}$, we solved for the optimal decision boundaries by setting all the Lagrange multipliers to zero and solving (126a). Depending upon the distribution, 126a) can be solved exactly or with a non-linear solver.

b) Entropy-constrained minimum distortion: The interval $U_{i}$ that the $i$-th user's observed value lies in is a discrete random variable with probability mass function given by $\boldsymbol{p}=\left(p_{k}: k=1, \ldots, K\right)$ and the entropy of $U_{i}$ is $H\left(U_{i}\right)=-\sum_{k=1}^{K} p_{k} \log _{2} p_{k}$. The total rate needed for the $N$ users to report their intervals is then

$$
R_{H o m S Q}(\ell) \triangleq \sum_{i=1}^{N} H\left(U_{i}\right)=N H(U)
$$

by the i.i.d. assumption of the sources and the homogeneity of the quantizers.

\section{Lemma 8.}

$$
\frac{\partial R_{H o m S Q}(\ell)}{\partial \ell_{k}}=N f_{k} \log _{2}\left(\frac{p_{k+1}}{p_{k}}\right)
$$

Proof:

$$
\begin{aligned}
\frac{\partial R_{H o m S Q}(\ell)}{\partial \ell_{k}} & =N \sum_{j=1}^{K} \frac{\partial}{\partial \ell_{k}} p_{j} \log \left(\frac{1}{p_{j}}\right) \\
& =N\left(\frac{\partial}{\partial \ell_{k}} p_{k} \log \left(\frac{1}{p_{k}}\right)+\frac{\partial}{\partial \ell_{k}} p_{k+1} \log \left(\frac{1}{p_{k+1}}\right)\right) \\
& =N\left(-f_{k}+f_{k} \log \left(\frac{1}{p_{k}}\right)+f_{k}-f_{k} \log \left(\frac{1}{p_{k+1}}\right)\right)
\end{aligned}
$$



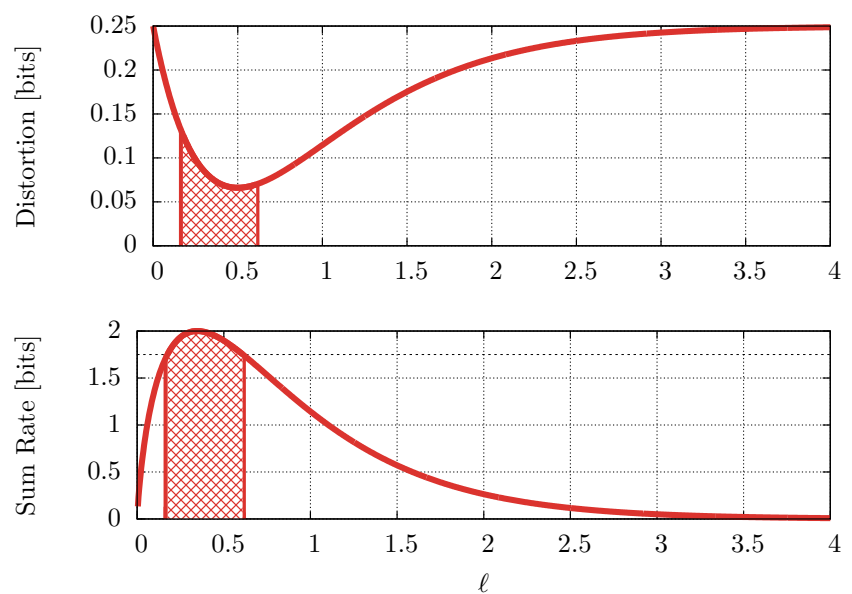

Fig. 8. Plots of $D(\ell)$ and $R(\ell)$ as functions of $\ell$. For $R(\ell) \leq 1.75$, the set of feasible $\ell$ is seen to be non-convex.

We now consider the problem of minimizing the distortion subject to an upper limit on the sum rate.

$$
\begin{array}{cl}
\underset{\ell}{\operatorname{minimize}} & D(\ell) \\
\text { subject to } & R_{H o m S Q}(\ell) \leq R_{0} \\
& \ell_{k-1} \leq \ell_{k} \quad k=1, \ldots, K
\end{array}
$$

In general, this problem is not convex. To see this, consider $X_{i} \sim \operatorname{Exp}(\lambda)$ and a single threshold $\ell$ (two intervals: $[0, \ell),[\ell, \infty)$ ). Fig. 8 shows a plot of $D(\ell)$ (top) and $R(\ell)$ (bottom) as $\ell$ is swept from $\inf \mathcal{X}$ to $\sup \mathcal{X}$. For $R_{0}=1.75$ bits, the range of infeasible $\ell$ is shown as a filled area under the rate and distortion curves and we see that the set of feasible $\ell$ is non-convex.

Theorem 11. If $\left\{\ell_{k}^{*}: k=0, \ldots, K\right\}$ is an optimal solution to (134), then there exists $\mu_{K}^{*} \geq 0$ for $k=1, \ldots, K$ and $\mu_{R} \geq 0$ such that

$$
\begin{gathered}
f_{k}\left[\frac{\left(F_{k+1}^{N}-F_{k}^{N}\right)\left(\ell_{k}^{*}-E_{k+1}\right)}{p_{k+1}}+\frac{\left(F_{k}^{N}-F_{k-1}^{N}\right)\left(E_{k}-\ell_{k}^{*}\right)}{p_{k}}-N F_{k}^{N-1}\left(E_{k}-E_{k+1}\right)+\mu_{R}^{*} N \log _{2}\left(\frac{p_{k+1}}{p_{k}}\right)\right] \\
-\mu_{k}^{*}+\mu_{k+1}^{*}=0 \\
\mu_{i}^{*}\left(\ell_{i-1}^{*}-\ell_{i}^{*}\right)=0 \text { and } \mu_{R}^{*}\left(R_{H o m S Q}\left(\ell^{*}\right)-R_{0}\right)=0 .
\end{gathered}
$$

Proof: The Lagrangian associated with this problem is

$$
L(\ell, \boldsymbol{\mu})=D(\ell)+\mu_{R}\left(R_{H o m S Q}(\ell)-r\right)+\sum_{k=1}^{K} \mu_{k}\left(\ell_{k-1}-\ell_{k}\right)
$$


Taking the derivative w.r.t. $\ell_{i}$ gives

$$
\frac{\partial L(\boldsymbol{\ell}, \boldsymbol{\mu})}{\partial \ell_{i}}=\frac{\partial D(\ell)}{\partial \ell_{i}}+\mu_{R} \frac{\partial R_{H o m S Q}(\ell)}{\partial \ell_{i}}-\mu_{i}+\mu_{i+1} .
$$

The result follows from setting the above equal to zero and complementary slackness.

Remark. Solving for the optimal entropy constrained quantizer is more difficult than solving for the minimum distortion quantizer. Depending upon the given values of $R_{0}$ and $K$, the decision boundaries may collapse and the associated Lagrange multipliers need no longer be identically zero. A general solution technique for (135) is beyond the scope of the present paper; generalizations to both Lloyd's and Max's algorithms for entropy constrained quantizer design are presented in [45].

We conclude with some observations about the rate-distortion curve for entropy-constrained quantizers. For a given $K$, suppose $\ell^{*}$ is a solution to 125. If $R_{0} \geq R_{H o m S Q}\left(\ell^{*}\right)$, then the rate constraint in 134) is not active and $\ell^{*}$ is also a solution to 134 for the same $K$. On the other hand, if $R_{0}<R\left(\ell^{*}\right)$ then the rate constraint in 134 is active and $\ell^{*}$ is infeasible for [134] [45]. Next, consider the rate-distortion curve for a $N$-level entropy-constrained quantizer and the sequence of rate-distortion points given by (125) for $K=1, \ldots, N$. These rate-distortion points all lie in the rate-distortion curve for the $N$-level entropy-constrained quantizer.

2) Heterogeneous Scalar Quantizers: It is somewhat intuitive to suppose that because the sources are i.i.d., the quantizers at each user should be identical. For symmetric functions (e.g., max), Misra et al. consider only the design of the quantizer for a single user [34]. When the function is not symmetric (e.g., arg max as in our case), the assumption of HomSQ is in fact not true.

Theorem 12. For an optimal HomSQ $\ell^{*}$ that achieves a distortion $D\left(\ell^{*}\right)$, there exists a HetSQ that achieves the same distortion with rate

$$
R_{H e t S Q}(\ell)=(N-2) H(U)+\delta
$$

where

$$
\delta=\sum_{k=1}^{K} p_{k} \log \frac{1}{\left(p_{k-1}+p_{k}\right)\left(p_{k}+p_{k+1}\right)} \leq 2 H(U)
$$

and $p_{0}=0$ and $p_{K+1}=0$.

Proof: We think of HomSQ as approximating the continous distribution with a discrete one and then losslessly computing the arg max of the quantization bin indices. This is exactly the problem considered in Section IV] From Theorem 2, we know that fewer than $R_{H o m S Q}(\ell)$ bits are needed to enable the CEO to losslessly determine $\arg$ max of the bin indices. In the proof of Theorem 2, a code is constructed by coloring the vertices of the associated characteristic graphs for each user and entropy coding the vertex colors. The rate savings comes by allowing a pair of consecutive bin indices for a user to be assigned the same color, provided the pair of indices are assigned different colors for every other user. We can compute the colors directly, by observing that if a pair of consecutive bin indices are being assigned the same color we are merging the underlying bins into one larger bin for that user only. 


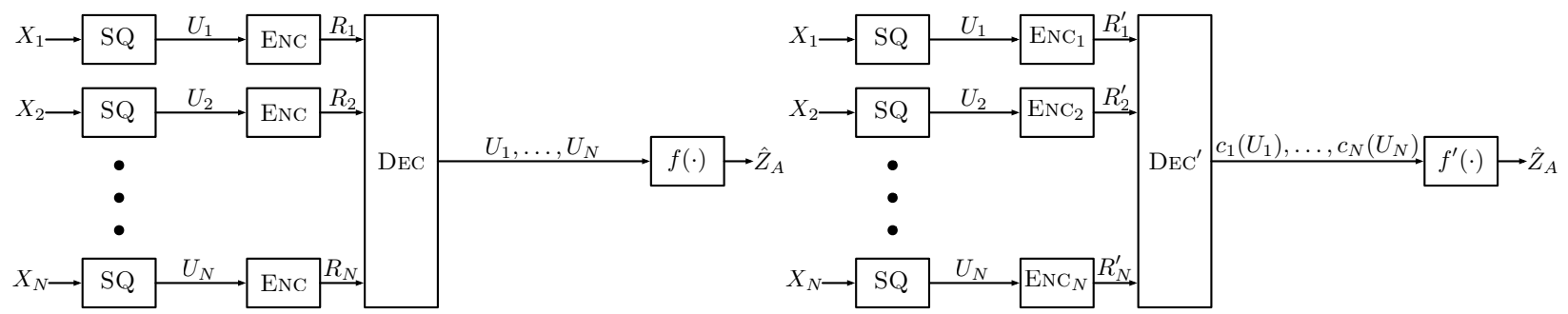

(a) HomSQ

(b) Alternative HomSQ

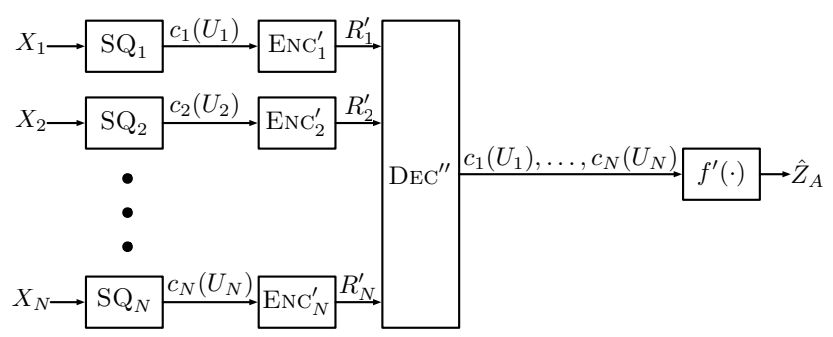

(c) HetSQ

Fig. 9. Block diagram of possible scalar quantizers for $\arg \max$. For HomSQ (a) $U_{1}, \ldots, U_{N}$ are i.i.d. and the encoder is the same for each user. In the alternative HomSQ scheme (b) the reduction in rate comes from coloring the vertices in a characteristic graph associated with each source. In general, this graph is different for each user and therefore the encoder will be different for each user. Finally, by having the quantization operation at each source determine the "vertex color", $U_{1}^{\prime}, \ldots, U_{N}^{\prime}$ are independent but not identically distributed.

Remark. As was shown in Theorem 7 , the total rate savings for losslessly determinging the arg max of a discrete distribution is at most 2 bits. Therefore, the rate savings of HetSQs versus HomSQs is also at most 2 bits and the savings per user goes to zero as the number of users is increased.

For HetSQ, when $N=2$ and $K=2$ only one of the sources is sending back a bit. We can use results from rate-distortion for the $\operatorname{Bernoulli}(p)$ source with Hamming distortion to trace out the low-rate/high-distortion segment of the trade-off curve.

Lemma 9. The expected value of the estimator when a lossy source code is used to communicate the output HetSQ for $N=2$ and $K=2$ to the CEO is given by

$$
\mathbb{E}\left[X_{\hat{Z}_{A}}\right]=(1-\hat{p}) \mathbb{E}[X]+\hat{p}\left(D_{H} \mathbb{E}[X \mid X \leq \ell]+\left(1-D_{H}\right) \mathbb{E}[X \mid \ell \leq X]\right)
$$

where

$$
\hat{p}=\frac{p_{2}-D_{H}}{1-2 D_{H}}
$$

and the rate is given by

$$
R\left(\ell, D_{H}\right)= \begin{cases}h_{2}\left(p_{2}\right)-h_{2}\left(D_{H}\right) & D_{H} \leq \min \left\{p_{2}, 1-p_{2}\right\} \\ 0 & D_{H}>\min \left\{p_{2}, 1-p_{2}\right\}\end{cases}
$$




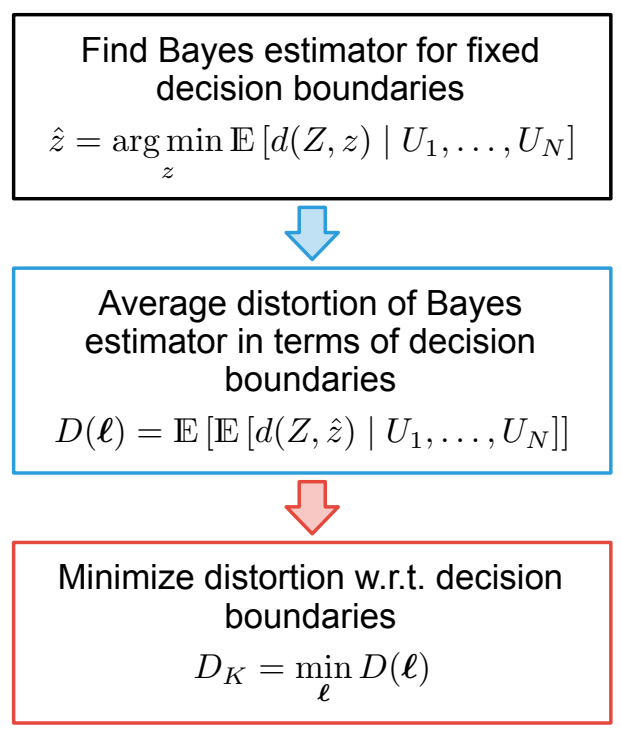

Fig. 10. Method utilized to design the scalar quantizers.

Proof: We assume that user 1 is sending the single indicator bit to the CEO w.l.o.g. and model this as a $\operatorname{Bernoulli}\left(p_{2}\right)$ source with $p_{2}=\mathbb{P}\left(X_{1} \geq \ell\right)$ and Hamming distortion $D_{H}$. The rate-distortion function for this subproblem is given by 142 . The test channel that achieves this is a binary symmetric channel $(\mathrm{BSC})\left(D_{H}\right)$ with input $\hat{X} \sim \operatorname{Bernoulli}(\hat{p})$. From this we obtain an expression for the joint probability mass function (pmf) $\mathbb{P}(X=x, \hat{X}=\hat{x})$ from which we can derive 140 .

Remark. Observer that for $D_{H}=0$, we obtain the same expression as (119) for $N=2$ and $K=2$ and for $D_{H}=\min \left\{p_{2}, 1-p_{2}\right\}$, we get $\mathbb{E}\left[X_{\hat{Z}_{A}}\right]=\mathbb{E}[X]$.

\section{Optimal HetSQ for $N=2$ Users}

Having considered SQs as an acheivable scheme for lossy determination of the arg max of a set of distributed sources, we investigate the use of SQs for the scenarios where the max and the pair $(\arg \max , \max )$ need to be determined. As was shown in the previous section, the assumption of homogeneity of the quantizers leads to suboptimal performance for arg max. For the other two functions, we will immediately consider the design of HetSQs.

We begin by formally stating the design process that was used implicitly in the previous section, which is depicted in Fig. 10

Let $X_{i}$ represent the source value of user $i, i \in\{1, \ldots, N\}$. The quantizer for user $i$ breaks its support $\mathcal{X}$ into $K$ intervals indexed by $k_{i} \in\{0, \ldots, K\}$

$$
\mathcal{X}=\bigcup_{k_{i}=1}^{K} \mathcal{L}_{i, k_{i}}, \quad \mathcal{L}_{i, k_{i}}=\left[\ell_{i, k_{i}-1}, \ell_{i, k_{i}}\right]
$$


Let $U_{i}$ be the index of the quantization interval user $i$ 's source value $X_{i}$ is in (i.e., $U_{i}=k_{i}$ if $X_{i} \in\left[\ell_{i, k_{i}-1}, \ell_{i, k_{i}}\right]$ ) and let $\mathbf{U}=\left(U_{i}: i \in 1, \ldots, N\right)$.

First, the Bayes estimator $\hat{z}(\mathbf{U})$ which minimizes the expected distortion given the quantization indices $\mathbf{U}$ is found by solving

$$
\hat{z}(\mathbf{U})=\underset{\hat{z}}{\arg \min } \mathbb{E}\left[d(Z, \hat{z}) \mid U_{1}, \ldots, U_{N}\right]
$$

as a function of the decision boundaries $\ell=\left[\ell_{i, k}: k \in\{1, \ldots, K\}\right]$ and the common $\operatorname{CDF} F_{X}(x)$ of the sources. Next, the expected distortion of the Bayes estimator is expressed in terms of the decision boundaires levels and the distribution of the source

$$
D(\ell)=\mathbb{E}\left[\mathbb{E}\left[d(Z, \hat{z}) \mid U_{1}, \ldots, U_{N}\right]\right]
$$

Finally, this expression is numerically optimized to yield the minimum distortion HetSQ $\ell$ for a given number of quantization levels $K$

$$
D_{K}=\min _{\ell} D(\ell)
$$

The resulting sum-rate distortion pair for each $K$ is then $\left(R_{K}, D_{K}\right)$ where

$$
R_{K}=-\sum_{i=1}^{N} \sum_{k_{1}=1}^{K} \mathbb{P}\left(U_{i}=k_{i}\right) \log _{2} \mathbb{P}\left(U_{i}=k_{i}\right)
$$

which assumes that the quantization indices $U_{i}$ will be block Huffman coded so as to approach a rate equal to their entropy. Note that we do not consider the more complicated case of entropy constrained scalar quantization as the simpler minimum distortion quantizers already require calculations that are somewhat dense, and also, for the sources of interest, yield rate distortion tradeoffs close to the fundamental limits.

For the case of $N=2$, we provide expressions for Bayes estimator and the average distortion of the Bayes estimator as a function of the quantizer parameters $\ell$. In Section V-D we numerically perform the optimization 146) for the case of an assumed distribution.

Theorem 13. The optimal Bayes estimator for the two-user arg max problem is

$$
\hat{z}\left(U_{1}, U_{2}\right)= \begin{cases}1 & \text { if } \mathbb{E}\left[X_{1} \mid U_{1}\right] \geq \mathbb{E}\left[X_{2} \mid U_{2}\right] \\ 2 & \text { if } \mathbb{E}\left[X_{1} \mid U_{1}\right]<\mathbb{E}\left[X_{2} \mid U_{2}\right]\end{cases}
$$


and the expected distortion when using the optimal Bayes estimator is given by

$$
\begin{aligned}
D(\ell) & =\mathbb{E}\left[d\left(\left(X_{1}, X_{2}\right), \hat{z}\left(U_{1}, U_{2}\right)\right)\right] \\
& =\sum_{\left(k_{1}, k_{2}\right) \in \mathbf{Z}_{01}} \int_{\max \left(\ell_{1, k_{1}-1}, \ell_{2, k_{2}-1}\right)}^{\ell_{2, k_{2}}} z f_{X}(z)\left[2 F_{X}(z)-F_{X}\left(\ell_{2, k_{2}-1}\right)-F_{X}\left(\ell_{1, k_{1}-1}\right)\right] \mathrm{d} z \\
& +\sum_{\left(k_{1}, k_{2}\right) \in \mathbf{Z}_{01}} \int_{\ell_{2, k_{2}}}^{\ell_{1, k_{1}}} z f_{X}(z)\left[F_{X}\left(\ell_{2, k_{2}}\right)-F_{X}\left(\ell_{2, k_{2}-1}\right)\right] \mathrm{d} z \\
& -\sum_{\left(k_{1}, k_{2}\right) \in \mathbf{Z}_{01}} \max \left(\mathbb{E}\left[X_{1} \mid U_{1}=k_{1}\right], \mathbb{E}\left[X_{2} \mid U_{2}=k_{2}\right]\right) \mathbb{P}\left(U_{1}=k_{1}\right) \mathbb{P}\left(U_{2}=k_{2}\right) \\
& +\sum_{\left(k_{1}, k_{2}\right) \in \mathbf{Z}_{02}} \int_{\max \left(\ell_{1, k_{1}-1}, \ell_{2, k_{2}-1}\right)}^{\ell_{1, k_{1}}} f_{X}(z)\left[2 F_{X}(z)-F_{X}\left(\ell_{1, k_{1}-1}\right)-F_{X}\left(\ell_{2, k_{2}-1}\right)\right] \mathrm{d} z \\
& +\sum_{\left(k_{1}, k_{2}\right) \in \mathbf{Z}_{02}} \int_{\ell_{1, k_{1}}}^{\ell_{2, k_{2}}} z f_{X}(z)\left[F_{X}\left(\ell_{1, k_{1}}\right)-F_{X}\left(\ell_{1, k_{1}-1}\right)\right] \mathrm{d} z \\
& -\sum_{\left(k_{1}, k_{2}\right) \in \mathbf{Z}_{02}} \max \left(\mathbb{E}\left[X_{1} \mid X_{1} \in \mathcal{L}_{1, k_{1}}\right], \mathbb{E}\left[X_{2} \mid X_{2} \in \mathcal{L}_{2, k_{2}}\right]\right) \mathbb{P}\left(U_{1}=k_{1}\right) \mathbb{P}\left(U_{2}=k_{2}\right)
\end{aligned}
$$

where

$$
\begin{aligned}
& \mathbf{Z}_{01}=\left\{k_{1}, k_{2}: \max \left(\ell_{1, k_{1}-1}, \ell_{2, k_{2}-1}\right) \leq \ell_{2, k_{2}} \leq \ell_{1, k_{1}}\right\} \\
& \mathbf{Z}_{02}=\left\{k_{1}, k_{2}: \max \left(\ell_{1, k_{1}-1}, \ell_{2, k_{2}-1}\right) \leq \ell_{1, k_{1}} \leq \ell_{2, k_{2}}\right\} .
\end{aligned}
$$

Proof: See Appendix A7

We can repeat a similar procedure for case where the CEO is interested in estimating max of two distributed sources.

Theorem 14. The optimal Bayes estimator for $Z_{M}=\max \left(X_{1}, X_{2}\right)$ is given by

$$
\hat{z}\left(U_{1}, U_{2}\right)= \begin{cases}\hat{z}_{1}^{*} & \text { if } \ell_{1, U_{1}-1} \geq \ell_{2, U_{2}} \\ \hat{z}_{2}^{*} & \text { if } \ell_{2, U_{2}-1} \geq \ell_{1, U_{1}} \\ \hat{z}_{01}^{*} & \text { if } \max \left(\ell_{2, U_{2}-1}, \ell_{1, U_{1}-1}\right)<\ell_{2, U_{2}} \leq \ell_{1, U_{1}} \\ \hat{z}_{02}^{*} & \text { if } \max \left(\ell_{1, U_{1}-1}, \ell_{2, U_{2}-1}\right)<\ell_{1, U_{1}} \leq \ell_{2, U_{2}}\end{cases}
$$

where

$$
\begin{gathered}
\hat{z}_{1}^{*}= \begin{cases}\operatorname{sol}\left\{z: F_{X}\left(\ell_{1, U_{1}}\right)=F_{X}(z)+z f_{X}(z), 2 f_{X}(z)+z f_{X}^{\prime}(z) \geq 0\right\} & z \in \mathcal{L}_{1, U_{1}} \\
\ell_{1, U_{1}-1} & \text { otherwise }\end{cases} \\
\hat{z}_{2}^{*}= \begin{cases}\operatorname{sol}\left\{z: F_{X}\left(\ell_{2, U_{2}}\right)=F_{X}(z)+z f_{X}(z), 2 f_{X}(z)+z f_{X}^{\prime}(z) \geq 0\right\} & z \in \mathcal{L}_{2, U_{2}} \\
\ell_{2, U_{2}-1} & \text { otherwise }\end{cases} \\
\hat{z}_{01}^{*}= \begin{cases}\hat{z}_{11}^{*} & w_{11}\left(\hat{z}_{11}^{*}\right) \geq w_{12}\left(\hat{z}_{12}^{*}\right) \\
\hat{z}_{12}^{*} & \text { otherwise. }\end{cases}
\end{gathered}
$$




$$
\begin{aligned}
& \hat{z}_{02}^{*}= \begin{cases}\hat{z}_{21}^{*} & w_{21}\left(\hat{z}_{21}^{*}\right) \geq w_{22}\left(\hat{z}_{22}^{*}\right) \\
\hat{z}_{22}^{*} & \text { otherwise }\end{cases} \\
& w_{11}(z)=z\left[1-F_{X \mid X \in \mathcal{L}_{1, U_{1}}}(z) F_{X \mid X \in \mathcal{L}_{2, U_{2}}}(z)\right] \\
& w_{12}(z)=z\left[1-F_{X \mid X \in \mathcal{L}_{1, U_{1}}}(z)\right] \\
& \hat{z}_{11}^{*}= \begin{cases}\operatorname{sol}\left\{z: w_{11}^{\prime}(z)=0, w_{11}^{\prime \prime}(z) \leq 0\right\} & \max \left(\ell_{1, U_{1}-1}, \ell_{2, U_{2}-1}\right) \leq z \leq \ell_{2, U_{2}} \\
\max \left(\ell_{1, U_{1}-1}, \ell_{2, U_{2}-1}\right) & \text { otherwise }\end{cases} \\
& \hat{z}_{12}^{*}= \begin{cases}\operatorname{sol}\left\{z: F_{X}\left(\ell_{1, U_{1}}\right)=F_{X}(z)+z f_{X}(z), 2 f_{X}(z)+z f_{X}^{\prime}(z) \geq 0\right\} & \ell_{2, U_{2}} \leq z \leq \ell_{1, U_{1}} \\
\ell_{2, U_{2}} & \text { otherwise }\end{cases} \\
& w_{21}(z)=z\left[1-F_{X \mid X \in \mathcal{L}_{1, U_{1}}}(z) F_{X \mid X \in \mathcal{L}_{2, U_{2}}}(z)\right] \\
& w_{22}(z)=z\left[1-F_{X \mid X \in \mathcal{L}_{2, U_{2}}}(z)\right] \\
& \hat{z}_{21}^{*}= \begin{cases}\operatorname{sol}\left\{z: w_{21}^{\prime}(z)=0, w_{21}^{\prime \prime}(z) \leq 0\right\} & \max \left(\ell_{1, U_{1}-1}, \ell_{2, U_{2}-1}\right) \leq z \leq \ell_{1, U_{1}} \\
\max \left(\ell_{1, U_{1}-1}, \ell_{2, U_{2}-1}\right) & \text { otherwise }\end{cases} \\
& \hat{z}_{22}^{*}= \begin{cases}\operatorname{sol}\left\{z: F_{X}\left(\ell_{2, U_{2}}\right)=F_{X}(z)+z f_{X}(z), 2 f_{X}(z)+z f_{X}^{\prime}(z) \geq 0\right\} & \ell_{1, U_{1}} \leq z \leq \ell_{2, U_{2}} \\
\ell_{1, U_{1}} & \text { otherwise }\end{cases}
\end{aligned}
$$

Furthermore, the expected distortion when using the optimal Bayes estimator is given by

$$
\begin{aligned}
D(\ell) & =\mathbb{E}\left[d\left(\left(X_{1}, X_{2}\right), \hat{z}\right)\right] \\
& =\sum_{\left(k_{1}, k_{2}\right) \in \mathbf{Z}_{1}}\left[\int_{\ell_{1, k_{1}-1}}^{\ell_{1, k_{1}}} x f_{X}(x) \mathrm{d} x-\hat{z}_{1}^{*}\left[F_{X}\left(\ell_{1, k_{1}}\right)-F_{X}\left(\hat{z}_{1}^{*}\right)\right]\right]\left[F_{X}\left(\ell_{2, k_{2}}\right)-F_{X}\left(\ell_{2, k_{2}-1}\right)\right] \\
& +\sum_{\left(k_{1}, k_{2}\right) \in \mathbf{Z}_{2}}\left[\int_{\ell_{2, k_{2}-1}}^{\ell_{2, k_{2}}} x f_{X}(x) \mathrm{d} x-\hat{z}_{2}^{*}\left[F_{X}\left(\ell_{2, k_{2}}\right)-F_{X}\left(\hat{z}_{2}^{*}\right)\right]\right]\left[F_{X}\left(\ell_{1, k_{1}}\right)-F_{X}\left(\ell_{1, k_{1}-1}\right)\right] \\
& +\sum_{\left(k_{1}, k_{2}\right) \in \mathbf{Z}_{01}} \int_{\max \left(\ell_{1, k_{1}-1}, \ell_{2, k_{2}-1}\right)}^{\ell_{2, k_{2}}} z f_{X}(z)\left[2 F_{X}(z)-F_{X}\left(\ell_{2, k_{2}-1}\right)-F_{X}\left(\ell_{1, k_{1}-1}\right)\right] \mathrm{d} z \\
& +\sum_{\left(k_{1}, k_{2}\right) \in \mathbf{Z}_{01}} \int_{\ell_{2, k_{2}}}^{\ell_{1, k_{1}}} z f_{X}(z)\left[F_{X}\left(\ell_{2, k_{2}}\right)-F_{X}\left(\ell_{2, k_{2}-1}\right)\right] \mathrm{d} z \\
& -\sum_{\left(k_{1}, k_{2}\right) \in \mathbf{Z}_{01}} \max \left\{w_{11}\left(\hat{z}_{11}^{*}\right), w_{12}\left(\hat{z}_{12}^{*}\right)\right\}\left[F_{X}\left(\ell_{1, k_{1}}\right)-F_{X}\left(\ell_{1, k_{1}-1}\right)\right]\left[F_{X}\left(\ell_{2, k_{2}}\right)-F_{X}\left(\ell_{2, k_{2}-1}\right)\right] \\
& +\sum_{\left(k_{1}, k_{2}\right) \in \mathbf{Z}_{02}} \int_{\max \left(\ell_{1, k_{1}-1}, \ell_{2, k_{2}-1}\right)}^{\ell_{1, k_{1}}} z f_{X}(z)\left[2 F_{X}(z)-F_{X}\left(\ell_{1, k_{1}-1}\right)-F_{X}\left(\ell_{2, k_{2}-1}\right)\right] \mathrm{d} z \\
& +\sum_{\left(k_{1}, k_{2}\right) \in \mathbf{Z}_{02}} \int_{\ell_{1, k_{1}}}^{\ell_{2, k_{2}}} z f_{X}(z)\left[F_{X}\left(\ell_{1, k_{1}}\right)-F_{X}\left(\ell_{1, k_{1}-1}\right)\right] \mathrm{d} z \\
& -\sum_{\left(k_{1}, k_{2}\right) \in \mathbf{Z}_{02}} \max \left\{w_{21}\left(\hat{z}_{21}^{*}\right), w_{22}\left(\hat{z}_{22}^{*}\right)\right\}\left[F_{X}\left(\ell_{1, k_{1}}\right)-F_{X}\left(\ell_{1, k_{1}-1}\right)\right]\left[F_{X}\left(\ell_{2, k_{2}}\right)-F_{X}\left(\ell_{2, k_{2}-1}\right)\right]
\end{aligned}
$$


where

$$
\begin{aligned}
\mathbf{Z}_{1} & =\left\{\left(k_{1}, k_{2}\right): \ell_{2, k_{2}} \leq \ell_{1, k_{1}-1}\right\} \\
\mathbf{Z}_{2} & =\left\{\left(k_{1}, k_{2}\right): \ell_{1, k_{1}} \leq \ell_{2, k_{2}-1}\right\} \\
\mathbf{Z}_{01} & =\left\{k_{1}, k_{2}: \max \left(\ell_{1, k_{1}-1}, \ell_{2, k_{2}-1}\right) \leq \ell_{2, k_{2}} \leq \ell_{1, k_{1}}\right\} \\
\mathbf{Z}_{02} & =\left\{k_{1}, k_{2}: \max \left(\ell_{1, k_{1}-1}, \ell_{2, k_{2}-1}\right) \leq \ell_{1, k_{1}} \leq \ell_{2, k_{2}}\right\} .
\end{aligned}
$$

Proof: See Appendix A8

Finally, we consider the design of HetSQs for the scenario where the CEO wishes to estimate both $Z_{M}=$ $\max \left(X_{1}, X_{2}\right)$ and $Z_{A}=\arg \max \left(X_{1}, X_{2}\right)$.

Theorem 15. The optimal Bayes estimator for $\left(Z_{M}, Z_{A}\right)=\left(\max \left(X_{1}, X_{2}\right)\right.$, $\left.\arg \max \left(X_{1}, X_{2}\right)\right)$ is given by

$$
\hat{z}^{*}\left(U_{1}, U_{2}\right)= \begin{cases}\left(\hat{z}_{1}^{*}, 1\right) & \text { if } \ell_{1, U_{1}-1} \geq \ell_{2, U_{2}} \\ \left(\hat{z}_{2}^{*}, 2\right) & \text { if } \ell_{2, U_{2}-1} \geq \ell_{1, U_{1}} \\ \left(\hat{z}_{0}^{*}, \hat{i}_{0}^{*}\right) & \text { if } \max \left(\ell_{2, U_{2}-1}, \ell_{1, U_{1}-1}\right) \leq \min \left(\ell_{1, U_{1}}, \ell_{2, U_{2}}\right) \leq \max \left(\ell_{1, U_{1}}, \ell_{2, U_{2}}\right)\end{cases}
$$

where

$$
\begin{aligned}
& \hat{z}_{1}^{*}= \begin{cases}\operatorname{sol}\left\{z: F_{X}\left(\ell_{1, U_{1}}\right)=F_{X}(z)+z f_{X}(z), 2 f_{X_{1}}(z)+z f_{X}^{\prime}(z) \geq 0\right\} & z \in \mathcal{L}_{1, U_{1}} \\
\ell_{1, U_{1}-1} & \text { otherwise }\end{cases} \\
& \hat{z}_{2}^{*}= \begin{cases}\operatorname{sol}\left\{z: F_{X}\left(\ell_{2, U_{2}}\right)=F_{X}(z)+z f_{X}(z), 2 f_{X_{2}}(z)+z f_{X_{2}}^{\prime}(z) \geq 0\right\} & z \in \mathcal{L}_{2, U_{2}} \\
\ell_{2, U_{2}-1} & \text { otherwise }\end{cases} \\
& \hat{z}_{0}^{*}= \begin{cases}\hat{z}_{1}^{*} & \text { if } w\left(\hat{z}_{1}^{*}\right) \geq w\left(\hat{z}_{2}^{*}\right) \\
\hat{z}_{2}^{*} & \text { otherwise. }\end{cases} \\
& \hat{i}_{0}^{*}= \begin{cases}1 & \text { if } w\left(\hat{z}_{1}^{*}\right) \geq w\left(\hat{z}_{2}^{*}\right) \\
2 & \text { otherwise }\end{cases} \\
& w(z)=z \frac{F_{X}\left(\ell_{\hat{i}, U_{\hat{i}}}\right)-F_{X}(z)}{F_{X}\left(\ell_{\hat{i}, U_{\hat{i}}}\right)-F_{X}\left(\ell_{\hat{i}, U_{\hat{i}}-1}\right)}
\end{aligned}
$$


Furthermore, the expected distortion when using the optimal Bayes estimator is given by

$$
\begin{aligned}
D(\ell) & =\mathbb{E}\left[d_{M, A}\left(\left(X_{1}, X_{2}\right),(\hat{z}, \hat{i})\right)\right] \\
& =\sum_{\left(k_{1}, k_{2}\right) \in \mathbf{Z}_{1}}\left[\int_{\ell_{1, k_{1}-1}}^{\ell_{1, k_{1}}} x f_{X}(x) \mathrm{d} x-\hat{z}_{1}^{*}\left(F_{X}\left(\ell_{1, k_{1}}\right)-F_{X}\left(\hat{z}_{1}^{*}\right)\right)\right]\left(F_{X}\left(\ell_{2, k_{2}}\right)-F_{X}\left(\ell_{2, k_{2}-1}\right)\right) \\
& +\sum_{\left(k_{1}, k_{2}\right) \in \mathbf{Z}_{2}}\left[\int_{\ell_{2, k_{2}-1}}^{\ell_{2, k_{2}}} x f_{X}(x) \mathrm{d} x-\hat{z}_{2}^{*}\left(F_{X}\left(\ell_{2, k_{2}}\right)-F_{X}\left(\hat{z}_{2}^{*}\right)\right)\right]\left(F_{X}\left(\ell_{1, k_{1}}\right)-F_{X}\left(\ell_{1, k_{1}-1}\right)\right) \\
& +\sum_{\left(k_{1}, k_{2}\right) \in \mathbf{Z}_{01}} \int_{\max \left(\ell_{1, k_{1}-1}, \ell_{2, k_{2}-1}\right)}^{\ell_{2, k_{2}}} z f_{X}(z)\left(2 F_{X}(z)-F_{X}\left(\ell_{2, k_{2}-1}\right)-F_{X}\left(\ell_{1, k_{1}-1}\right)\right) \mathrm{d} z \\
& +\sum_{\left(k_{1}, k_{2}\right) \in \mathbf{Z}_{01}} \int_{\ell_{2, k_{2}}}^{\ell_{1, k_{1}}} z f_{X}(z)\left(F_{X}\left(\ell_{2, k_{2}}\right)-F_{X}\left(\ell_{2, k_{2}-1}\right)\right) \mathrm{d} z \\
& -\sum_{\left(k_{1}, k_{2}\right) \in \mathbf{Z}_{01}} \max \left\{w\left(\hat{z}_{1}^{*}\right), w\left(\hat{z}_{2}^{*}\right)\right\}\left(F_{X}\left(\ell_{1, k_{1}}\right)-F_{X}\left(\ell_{1, k_{1}-1}\right)\left(F_{X}\left(\ell_{2, k_{2}}\right)-F_{X}\left(\ell_{2, k_{2}-1}\right)\right)\right. \\
& +\sum_{\left(k_{1}, k_{2}\right) \in \mathbf{Z}_{02}} \int_{\max \left(\ell_{1, k_{1}-1}, \ell_{2, k_{2}-1}\right)}^{\ell_{1, k_{1}}} z f_{X}(z)\left(2 F_{X}(z)-F_{X}\left(\ell_{1, k_{1}-1}\right)-F_{X}\left(\ell_{2, k_{2}-1}\right)\right) \mathrm{d} z \\
& +\sum_{\left(k_{1}, k_{2}\right) \in \mathbf{Z}_{02}} \int_{\ell_{1, k_{1}}}^{\ell_{2, k_{2}}} z f_{X}(z)\left(F_{X}\left(\ell_{1, k_{1}}\right)-F_{X}\left(\ell_{1, k_{1}-1}\right)\right) \mathrm{d} z \\
& -\sum_{\left(k_{1}, k_{2}\right) \in \mathbf{Z}_{02}} \max \left\{w\left(\hat{z}_{1}^{*}\right), w\left(\hat{z}_{2}^{*}\right)\right\}\left(F_{X}\left(\ell_{1, k_{1}}\right)-F_{X}\left(\ell_{1, k_{1}-1}\right)\right)\left(F_{X}\left(\ell_{2, k_{2}}\right)-F_{X}\left(\ell_{2, k_{2}-1}\right)\right)
\end{aligned}
$$

where

$$
\begin{aligned}
\mathbf{Z}_{1} & =\left\{\left(k_{1}, k_{2}\right): \ell_{2, k_{2}} \leq \ell_{1, k_{1}-1}\right\} \\
\mathbf{Z}_{2} & =\left\{\left(k_{1}, k_{2}\right): \ell_{1, k_{1}} \leq \ell_{2, k_{2}-1}\right\} \\
\mathbf{Z}_{01} & =\left\{k_{1}, k_{2}: \max \left(\ell_{1, k_{1}-1}, \ell_{2, k_{2}-1}\right) \leq \ell_{2, k_{2}} \leq \ell_{1, k_{1}}\right\} \\
\mathbf{Z}_{02} & =\left\{k_{1}, k_{2}: \max \left(\ell_{1, k_{1}-1}, \ell_{2, k_{2}-1}\right) \leq \ell_{1, k_{1}} \leq \ell_{2, k_{2}}\right\} .
\end{aligned}
$$

Proof: See Appendix A9

We conclude by breifly commenting on the subtle difference between between the design of the Bayes estimator for estimating the max (Theorem 14 and estimating the pair (max, arg max) (Theorem 13). For the case of estimating the max, the Bayes estimator is given by

$$
\underset{z}{\arg \max } z \mathbb{P}\left(\max \left\{X_{1}, X_{2}\right\} \geq z \mid X_{1} \in \mathcal{L}_{1, U_{1}}, X_{2} \in \mathcal{L}_{2, U_{2}}\right)
$$

while for the case of estimating the pair ( $\max , \arg \max )$, the Bayes estimator is given by

$$
\underset{z, i}{\arg \max } z \mathbb{P}\left(X_{i} \geq z \mid X_{1} \in \mathcal{L}_{1, U_{1}}, X_{2} \in \mathcal{L}_{2, U_{2}}\right) .
$$

If $\mathcal{L}_{1, U_{1}} \cap \mathcal{L}_{2, U_{2}}=\varnothing$, the above expressions are identical becuse the CEO can identify the arg max with zero error. If, on the other hand, $\mathcal{L}_{1, U_{1}} \cap \mathcal{L}_{2, U_{2}} \neq \varnothing$ then the two expressions are different; in the first case, the objective function is a product of conditional CDFs and in the second case, the objective function is a conditional CDF. 


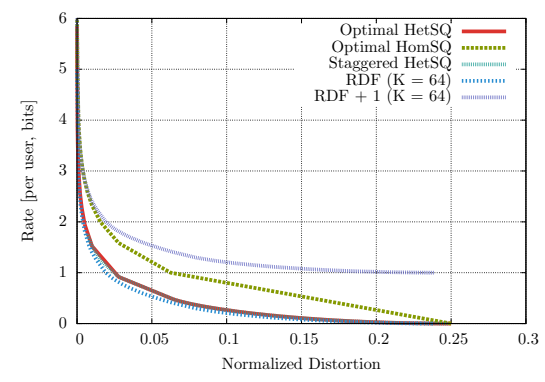

(a)

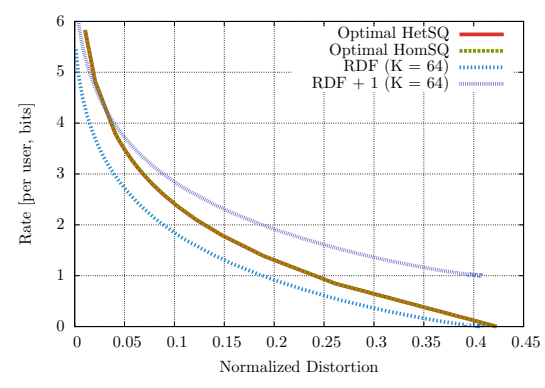

(b)

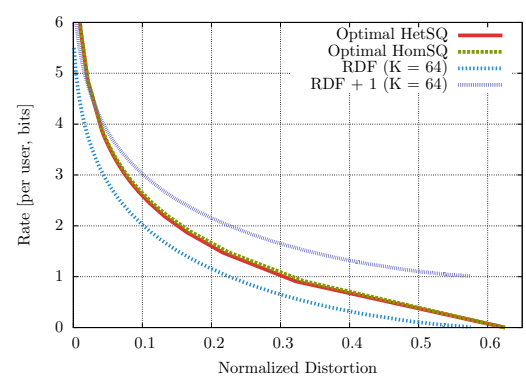

(c)

Fig. 11. Rate distortion tradeoff for numerically optimized HetSQ for two users with sources distributed Uniform( $(0,1)$ : (a) $\arg \max$; (b) max, and; (b) $(\arg \max , \max )$. The rate-distortion performance of HomSQ and HetSQ is compared to the rate-distortion function. For the purposes of comparison, we have included a trendline for the rate distortion function plus a bit.

For a fixed rate, the HetSQ for max should be able to acheive a lower distortion than the HetSQ for the pair $(\max , \arg \max )$.

\section{Examples}

In this section, we consider two different continuous distributions for the sources and compare the performance of HomSQ, homogeneous entropy-constrained scalar quantization (ECSQ), and HetSQ. We also show results for a discrete distribution in order to gauge the performance of the SQs relative to fundamental limit given by the rate-distortion function.

Example 5 (arg max quantizer with Uniform $(0,1)$ ). When $X_{1}, X_{2} \sim$ Uniform $(0,1)$, the Bayes' detector is

$$
\hat{z}\left(U_{1}, U_{2}\right)= \begin{cases}1 \quad \text { if } \frac{\ell_{1, U_{1}-1}+\ell_{1, U_{1}}}{2} \geq \frac{\ell_{2, U_{2}-1}+\ell_{2, U_{2}}}{2} \\ 2 \quad \text { if } \frac{\ell_{1, U_{1}-1}+\ell_{1, U_{1}}}{2}<\frac{\ell_{2, U_{2}-1}+\ell_{2, U_{2}}}{2}\end{cases}
$$

For $\left(U_{1}=k_{1}, U_{2}=k_{2}\right) \in \mathbf{Z}_{01}$, the expected distortion $\mathbb{E}\left[d\left(\left(X_{1}, X_{2}\right), \hat{z}\left(U_{1}, U_{2}\right)\right) \mid U_{1}, U_{2}\right] \mathbb{P}\left(U_{1}, U_{2}\right)$ is

$$
\begin{array}{r}
{\left.\left[\frac{2}{3} z^{3}-\frac{1}{2}\left(\ell_{1, k_{1}-1}+\ell_{2, k_{2}-1}\right) z^{2}\right]\right|_{\max \left(\ell_{1, k_{1}-1}, \ell_{2, k_{2}-1}\right)} ^{\ell_{2, k_{2}}}+\left.\frac{1}{2}\left(\ell_{2, k_{2}}-\ell_{2, k_{2}-1}\right) z^{2}\right|_{\ell_{2, k_{2}}} ^{\ell_{1, k_{1}}}} \\
-\left(\ell_{1, k_{1}}-\ell_{1, k_{1}-1}\right)\left(\ell_{2, k_{2}}-\ell_{2, k_{2}-1}\right) \max \left\{\frac{\ell_{1, k_{1}}+\ell_{1, k_{1}-1}}{2}, \frac{\ell_{2, k_{2}}+\ell_{2, k_{2}-1}}{2}\right\}
\end{array}
$$

For $\left(U_{1}=k_{1}, U_{2}=k_{2}\right) \in \mathbf{Z}_{02}$, the expected distortion $\mathbb{E}\left[d\left(\left(X_{1}, X_{2}\right), \hat{z}\left(U_{1}, U_{2}\right)\right) \mid U_{1}, U_{2}\right] \mathbb{P}\left(U_{1}, U_{2}\right)$ is

$$
\begin{gathered}
{\left.\left[\frac{2}{3} z^{3}-\frac{1}{2}\left(\ell_{1, k_{1}-1}+\ell_{2, k_{2}-1}\right) z^{2}\right]\right|_{\max \left(\ell_{1, k_{1}-1}, \ell_{2, k_{2}-1}\right)} ^{\ell_{1, k_{1}}}+\left.\frac{1}{2}\left(\ell_{2, k_{2}}-\ell_{2, k_{2}-1}\right) z^{2}\right|_{\ell_{1, k_{1}}} ^{\ell_{2, k_{2}}}} \\
-\left(\ell_{1, k_{1}}-\ell_{1, k_{1}-1}\right)\left(\ell_{2, k_{2}}-\ell_{2, k_{2}-1}\right) \max \left\{\frac{\left(\ell_{1, k_{1}}+\ell_{1, k_{1}-1}\right)}{2}, \frac{\left(\ell_{2, k_{2}}+\ell_{2, k_{2}-1}\right)}{2}\right\}
\end{gathered}
$$

Summing the above two expressions over $\mathbf{Z}_{01}$ and $\mathbf{Z}_{02}$ gives expression for the distortion when the CEO utilizes a Bayes estimator a function of quantization decision boundaries $\ell$ that are heterogeneous across users. We then numerically optimize this expression to obtain the rate-distortion paris $\left(R_{K}, D_{K}\right)$ for the given number $K$ of quantization bins. 
For the case of HomSQ, we have

$$
\ell_{k}^{*}=\frac{k}{K}, \mu_{k}^{*}=0 \quad k \in 1, \ldots, K
$$

as a solution to 126. As expected, the optimal quantizer for a uniform distribution is uniform. Substituting into the expressions for distortion and rate we obtain

$$
D_{K}=\frac{1}{6 K^{2}}, R_{K}=N \log _{2} K
$$

Fig. 11a shows the per user rate and the normalized distortion of both HomSQ, staggered HetSQ, and the optimal HetSQ along with a numerically computed approximation of the rate-distortion function for function arg max. Interestingly, while the approach of staggering HomSQ decision boundaries across users to effect potentially suboptimal HetSQ design, we observe here that the optimal HetSQ we have derived yields nearly identical performances, at least for the two user case under investigation. Here, a large improvement is achieved by passing between the HomSQ and HetSQ designs. Additionally, all of the 16, 32, and 64 level fundamental limits are right on top of one another and have already effectively converged to the continuous limit. Finally, we observe that the designed practical scalar scheme is right up against the fundamental overhead performance tradeoff.

Example 6 (Two user 2-level distributed HomSQ for max with Uniform $(0,1)$ ). For 2-level scalar quantizer, we set up the qunatizer parameters as follows

$$
\begin{array}{ll}
\mathcal{L}_{1,1}=\left[\ell_{1,0}, \ell_{1,1}\right], & \mathcal{L}_{1,2}=\left[\ell_{1,1}, \ell_{1,2}\right] \\
\mathcal{L}_{2,1}=\left[\ell_{2,0}, \ell_{2,1}\right], & \mathcal{L}_{2,2}=\left[\ell_{2,1}, \ell_{2,2}\right]
\end{array}
$$

where $\ell_{1,0}=\ell_{2,0}=0$ and $\ell_{1,2}=\ell_{2,2}=1$. We want to find $l_{1,1}$ and $l_{2,1}$ minimizing the expected distortion. For convenience, we analyze a homogeneous scalar quantizer which has same parameters between users. In this case, we set $\ell_{1,1}=\ell_{2,1}=\ell$. First, we solve for the Bayes estimator $\hat{z}\left(U_{1}, U_{2}\right)$ as a function of $\ell$. Based on the Theorem 14, we obtain the following expression for the Bayes estimator

$$
\hat{z}\left(U_{1}, U_{2}\right)= \begin{cases}\frac{\ell}{\sqrt{3}} & \text { if } U_{1}=U_{2}=1 \\ \max \left\{\frac{1}{2}, \ell\right\} & \text { if } U_{1} \neq U_{2} \\ \frac{2 \ell+\sqrt{4 \ell^{2}-6 \ell+3}}{3} & \text { if } U_{1}=U_{2}=2\end{cases}
$$

Next, we substitute the above expression in the expression for conditional expected distortion to obtain, then we 
solve an optimization problem.

$$
\begin{aligned}
& \mathbb{E}\left[d\left(\left(X_{1}, X_{2}\right), \hat{z}\left(U_{1}, U_{2}\right)\right) \mid U_{1}, U_{2}\right] \mathbb{P}\left(U_{1}, U_{2}\right)= \\
& \begin{cases}\frac{2(\sqrt{3}-1)}{3 \sqrt{3}} \ell^{3} & \text { if } U_{1}=U_{2}=1 \\
\frac{\left(1-2 \ell^{2}\right) \ell}{2} \mathbb{1}_{\ell \leq \frac{1}{2}}+(1-\ell)^{2} \ell \mathbb{1}_{\ell>\frac{1}{2}} & \text { if } U_{1} \neq U_{2} \\
\frac{-7 \ell^{3}+36 \ell^{2}-45 \ell+18-2\left(4 \ell^{2}-6 \ell+3\right)^{\frac{3}{2}}}{27} & \text { if } U_{1}=U_{2}=2 .\end{cases}
\end{aligned}
$$

Finally, we numerically optimize the expected distortion as a function $\ell$. For $0<l \leq \frac{1}{2}$, the average distortion is

$$
D(\ell)=\frac{2(\sqrt{3}-1)}{3 \sqrt{3}} \ell^{3}+\frac{\left(1-2 \ell^{2}\right) \ell}{2}+\frac{-7 \ell^{3}+36 \ell^{2}-45 \ell+18-2\left(4 \ell^{2}-6 \ell+3\right)^{\frac{3}{2}}}{27}
$$

which has a minimum value of 0.2204 at $\ell=0.5$. In $\frac{1}{2} \leq l<1$, the average distortion is

$$
D(\ell)=\frac{2(\sqrt{3}-1)}{3 \sqrt{3}} \ell^{3}+(1-\ell)^{2} \ell+\frac{-7 \ell^{3}+36 \ell^{2}-45 \ell+18-2\left(4 \ell^{2}-6 \ell+3\right)^{\frac{3}{2}}}{27}
$$

which has a minimum value of 0.1742 at $\ell=0.7257$. Therefore, we should to choose the HomSQ parameter $\ell=0.7257$ to attain a minimum distortion of 0.1742 .

Example 7 (Two user $K$-level Distributed Scalar Quantizer for max with Uniform $(0,1)$ ). When the two users' source is $\operatorname{Uniform}(0,1)$, an expected minimum distortion is

$$
\begin{aligned}
D(\ell) & =\sum_{\left(k_{1}, k_{2}\right) \in \mathbf{Z}_{1}} \begin{cases}\frac{\left(\ell_{1, k_{1}}^{2}-2 \ell_{1, k_{1}-1}^{2}\right)\left(\ell_{2, k_{2}}-\ell_{2, k_{2}-1}\right)}{4} & \ell_{1, k_{1}} \geq 2 \ell_{1, k_{1}-1} \\
\frac{\left(\ell_{1, k_{1}}-\ell_{1, k_{1}-1}\right)^{2}\left(\ell_{2, k_{2}}-\ell_{2, k_{2}-1}\right)}{2} & \ell_{1, k_{1}}<2 \ell_{1, k_{1}-1}\end{cases} \\
& +\sum_{\left(k_{1}, k_{2}\right) \in \mathbf{Z}_{2}}\left\{\begin{array}{ll}
\frac{\left(\ell_{1, k_{1}}-\ell_{1, k_{1}-1}\right)\left(\ell_{2, k_{2}}^{2}-2 \ell_{2, k_{2}-1}^{2}\right)}{4} & \ell_{2, k_{2}} \geq 2 \ell_{2, k_{2}-1} \\
& +\sum_{\left(\ell_{1}, k_{2}\right) \in \mathbf{Z}_{01}}\left[\left.\left(\frac{2}{3} \hat{z}_{01}^{3}-\frac{\left(\ell_{1, k_{1}-1}+\ell_{1, k_{1}-1}\right)}{2} \hat{z}_{01}^{2}\right)\right|_{\max \left\{\ell_{1, k_{1}-1}, \ell_{2, k_{2}-1}\right\}} ^{\ell_{2, k_{2}}}+\left.\frac{\left(\ell_{2, k_{2}}-\ell_{2, k_{2}-1}\right)}{2} \hat{z}_{01}^{2}\right|_{\ell_{2, k_{2}}} ^{\ell_{1, k_{1}}}\right.
\end{array}\right] \\
& -\sum_{\left(k_{1}, k_{2}\right) \in \mathbf{Z}_{01}}\left[\left(\ell_{1, k_{1}}-\ell_{1, k_{1}-1}\right)\left(\ell_{2, k_{2}}-\ell_{2, k_{2}-1}\right) \max \left\{w_{11}\left(\hat{z}_{11}^{*}\right), w_{12}\left(\hat{z}_{12}^{*}\right)\right\}\right] \\
+ & \sum_{\left(k_{1}, k_{2}\right) \in \mathbf{Z}_{02}}\left[\left.\left(\frac{2}{3} \hat{z}_{02}^{3}-\frac{\left(\ell_{1, k_{1}-1}+\ell_{2, k_{2}-1}\right)}{2} \hat{z}_{02}^{2}\right)\right|_{\max \left\{\ell_{1, k_{1}-1}, \ell_{2, k_{2}-1}\right\}} ^{\ell_{1, k_{1}}}+\left.\frac{\left(\ell_{1, k_{1}}-\ell_{1, k_{1}-1}\right)}{2} \hat{z}_{02}^{2}\right|_{\ell_{1, k_{1}}} ^{\ell_{2, k_{2}}}\right] \\
& -\sum_{\left(k_{1}, k_{2}\right) \in \mathbf{Z}_{02}}\left[\left(\ell_{1, k_{1}}-\ell_{1, k_{1}-1}\right)\left(\ell_{2, k_{2}}-\ell_{2, k_{2}-1}\right) \max \left\{w_{21}\left(\hat{z}_{21}^{*}\right), w_{22}\left(\hat{z}_{22}^{*}\right)\right\}\right]
\end{aligned}
$$

where $\hat{z}_{01}^{*}, w_{11}(\cdot), \hat{z}_{11}^{*}, w_{12}(\cdot), \hat{z}_{12}^{*}, \hat{z}_{02}^{*}, w_{21}(\cdot), \hat{z}_{21}^{*}, w_{22}(\cdot)$, and $\hat{z}_{22}^{*}$ are given by 154161 .

Fig. $11 \mathrm{~b}$ shows the per user rate and normalized distortion for HomSQ and HetSQ along with a numerically computed approximation of the rate-distortion function for estimating the max of two distributed users with sources 
distributed Uniform $(0,1)$. Numerically optimizing the expected distortion for HomSQ and HetSQ yields ratedistortion pairs $\left(R_{K}, D_{K}\right)$ that are nearly identical. The achievable SQ schemes are not particularly far from the fundamental limit, leaving only a small gain possible from a better designed scalar of vector quantizer.

Example 8 ( $\max$ and $\arg \max$ Quantizer with Uniform $(0,1)$ ). When two users' source is Uniform $(0,1)$, the expected minimum distortion in region $\left(U_{1}=k_{1}, U_{2}=k_{2}\right) \in \mathbf{Z}_{1}$ is

$$
\begin{aligned}
& D(\boldsymbol{\ell})=\sum_{\left(k_{1}, k_{2}\right) \in \mathbf{Z}_{1}} \begin{cases}\frac{\left(\ell_{1, k_{1}}^{2}-2 \ell_{1, k_{1}-1}^{2}\right)\left(\ell_{2, k_{2}}-\ell_{2, k_{2}-1}\right)}{4} & \ell_{1, k_{1}} \geq 2 \ell_{1, k_{1}-1} \\
\frac{\left(\ell_{1, k_{1}}-\ell_{1, k_{1}-1}\right)^{2}\left(\ell_{2, k_{2}}-\ell_{2, k_{2}-1}\right)}{2} & \ell_{1, k_{1}}<2 \ell_{1, k_{1}-1}\end{cases} \\
& +\sum_{\left(k_{1}, k_{2}\right) \in \mathbf{Z}_{2}} \begin{cases}\frac{\left(\ell_{1, k_{1}}-\ell_{1, k_{1}-1}\right)\left(\ell_{2, k_{2}}^{2}-2 \ell_{2, k_{2}-1}^{2}\right)}{4} & \frac{\ell_{2, k_{2}}}{2} \geq \ell_{2, k_{2}-1} \\
\frac{\left(\ell_{1, k_{1}}-\ell_{1, k_{1}-1}\right)\left(\ell_{2, k_{2}}-\ell_{2, k_{2}-1}\right)^{2}}{2} & \frac{\ell_{2, k_{2}}}{2}<\ell_{2, k_{2}-1}\end{cases} \\
& +\left.\sum_{\left(k_{1}, k_{2}\right) \in \mathbf{Z}_{01}}\left[\frac{2}{3} z^{3}-\frac{\left(\ell_{1, k_{1}-1}+\ell_{2, k_{2}-1}\right)}{2} z^{2}\right]\right|_{\max \left\{\ell_{1, k_{1}-1}, \ell_{2, k_{2}-1}\right\}} ^{\ell_{2, k_{2}}}+\left.\frac{\left(\ell_{2, k_{2}}-\ell_{2, k_{2}-1}\right)}{2} z^{2}\right|_{\ell_{2, k_{2}}} ^{\ell_{1, k_{1}}} \\
& -\sum_{\left(k_{1}, k_{2}\right) \in \mathbf{Z}_{01}}\left(\ell_{1, k_{1}}-\ell_{1, k_{1}-1}\right)\left(\ell_{2, k_{2}}-\ell_{2, k_{2}-1}\right) \max \left\{z_{11}^{*} \frac{\ell_{1, k_{1}}-z_{11}^{*}}{\ell_{1, k_{1}}-\ell_{1, k_{1}-1}}, z_{12}^{*} \frac{\ell_{2, k_{2}}-z_{12}^{*}}{\ell_{2, k_{2}}-\ell_{2, k_{2}-1}}\right\} \\
& +\left.\sum_{\left(k_{1}, k_{2}\right) \in \mathbf{Z}_{02}}\left[\frac{2}{3} z^{3}-\frac{\left(\ell_{1, k_{1}-1}+\ell_{2, k_{2}-1}\right)}{2} z^{2}\right]\right|_{\max \left\{\ell_{1, k_{1}-1}, \ell_{2, k_{2}-1}\right\}} ^{\ell_{1, k_{1}}}+\left.\frac{\left(\ell_{2, k_{2}}-\ell_{2, k_{2}-1}\right)}{2} z^{2}\right|_{\ell_{1, k_{1}}} ^{\ell_{2, k_{2}}} \\
& -\sum_{\left(k_{1}, k_{2}\right) \in \mathbf{Z}_{02}}\left(\ell_{1, k_{1}}-\ell_{1, k_{1}-1}\right)\left(\ell_{2, k_{2}}-\ell_{2, k_{2}-1}\right) \max \left\{z_{21}^{*} \frac{\ell_{1, k_{1}}-z_{21}^{*}}{\ell_{1, k_{1}}-\ell_{1, k_{1}-1}}, z_{22}^{*} \frac{\ell_{2, k_{2}}-z_{22}^{*}}{\ell_{2, k_{2}}-\ell_{2, k_{2}-1}}\right\}
\end{aligned}
$$

where

$$
\begin{aligned}
& z_{11}^{*}= \begin{cases}\frac{\ell_{1, k_{1}}}{2} & \text { if } \ell_{1, k_{1}} \geq 2 \ell_{1, k_{1}-1} \\
\ell_{1, k_{1}-1} & \text { otherwise }\end{cases} \\
& z_{12}^{*}= \begin{cases}\frac{\ell_{2, k_{2}}}{2} & \text { if } \ell_{2, k_{2}} \geq 2 \ell_{2, k_{2}-1} \\
\ell_{2, k_{2}-1} & \text { otherwise }\end{cases} \\
& z_{21}^{*}= \begin{cases}\frac{\ell_{1, k_{1}}}{2} & \text { if } \ell_{1, k_{1}} \geq 2 \ell_{1, k_{1}-1} \\
\ell_{1, k_{1}-1} & \text { otherwise }\end{cases} \\
& z_{22}^{*}= \begin{cases}\frac{\ell_{2, k_{2}}}{2} & \text { if } \ell_{2, k_{2}} \geq 2 \ell_{2, k_{2}-1} \\
\ell_{2, k_{2}-1} & \text { otherwise }\end{cases}
\end{aligned}
$$

Fig. 11c shows the per user rate and normalized distortion for HomSQ and HetSQ along with a numerically computed approximation of the rate-distortion function for estimating both the arg max and max of two distributed users with sources distributed Uniform $(0,1)$. Unlike the previous example (estimating just the max), we do observe that HetSQ has a better performance than HomSQ although the improvement is not as marked as for estimating $\arg \max$. 


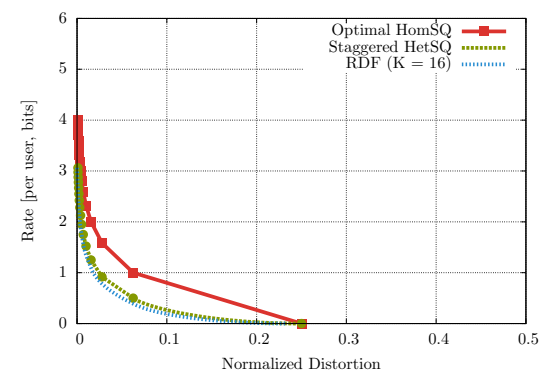

(a)

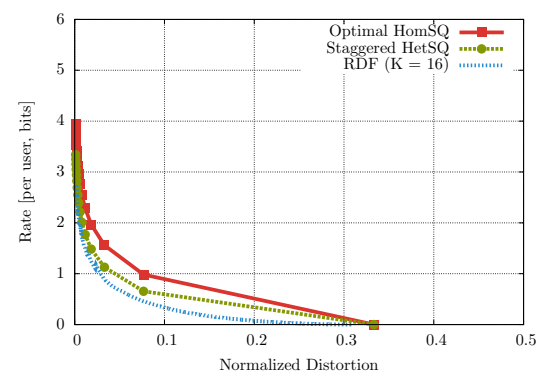

(b)

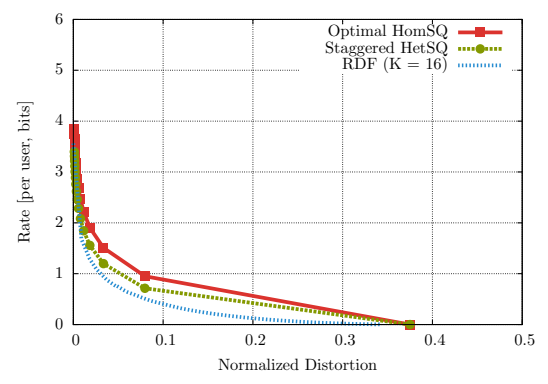

(c)

Fig. 12. Comparison of the rate-distortion tradeoff for HomSQ vs. HetSQ for: (a) $N=2$ users; (b) $N=3$ users, and; (c) $N=4$ users.

Example $9(\arg \max \mathrm{SQ}$ for $N>2$ Uniform $(0,1))$. We now consider the design of a HomSQ for estimating the $\arg \max$ from $N>2$ sources i.i.d. Uniform $(0,1)$. From 119 we obtain the following expression for the expected distortion

$$
D(\ell)=\frac{N}{N+1}-\sum_{j=1}^{K} \frac{\left(\ell_{j-1}+\ell_{j}\right)\left(\ell_{j}^{N}-\ell_{j-1}^{N}\right)}{2}
$$

from which we solve for optimal quantizer parameter $\ell^{*}$

$$
\ell_{j-1}^{* N-1}=\frac{\ell_{j+1}^{* N}-\ell_{j-1}^{* N}}{N\left(\ell_{j+1}^{*}-\ell_{j-1}^{*}\right)} .
$$

Fig. 12 shows the per user rate and normalized distortion for HomSQ and the staggered HetSQ derived from the optimal HomSQ for estimating the arg max of a collection of distributed users with sources i.i.d. Uniform $(0,1)$. The left subplot is for $N=2$ users, the middle subplot for $N=4$ users, and $N=8$ users. We observe immediately that the performance gains of the staggered HetSQ over HomSQ diminish as the number of users increases. Additionally, while the zero rate distortion is increasing in the number of users, we observe that the required rate per user to acheive a specified normalized distortion is non-montonic in the number of users. For example, fixing $D=0.01$ we observe per user rate for HomSQ is 2.32 bits for $N=2,2.32$ bits for $N=4$, and 1.86 bits for $N=8$. The per user rate for HetSQ is 1.52 bits for $N=2,1.95$ bits for $N=4$, and 1.71 bits for $N=8$.

Computing the rate distortion bounds becomes computationally expensive for a larger number of users $N$; however, we can investigate the scaling behavior of the presented achievable schemes for a wider range of $N$. We see in Fig. 13 that there is very little difference between the curves for $N=2$ and $N=4$, which matches with the behavior observed in Fig. 7. For larger values of $N$, we see that the per-user rate required to obtain a given distortion rapidly decreases with $N$. This proves in turn that the rate distortion function must also posses this property.

Our investigation of the rate distortion tradeoff for the CEO to compute the extremization functions in a lossy manner was motivated by the minimal rate savings shown in Section IV Shown in Table III are the rate savings of SQ for a small increase in tolerable distortion when the sources are distributed Uniform $(0,1)$. We see an average savings of about $43 \%$ accoss SQ type and number of users. We conclude that by incurring small increase in 


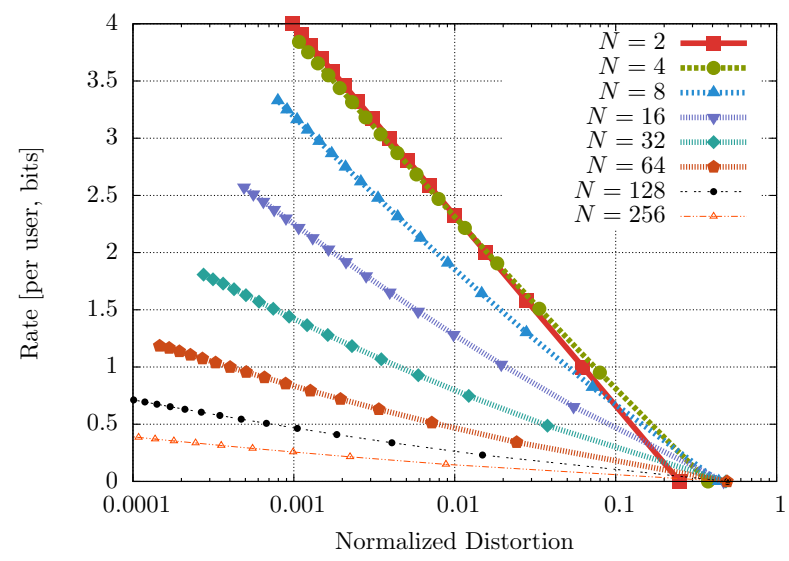

Fig. 13. Comparison of the rate-distortion tradeoff for optimal HomSQ as the number of users is increased.

\begin{tabular}{lcccccccc}
\hline & $N=2$ & $N=4$ & $N=8$ & $N=16$ & $N=32$ & $N=64$ & $N=128$ & $N=256$ \\
\cline { 2 - 7 } optimal HomSQ & $41.72 \%$ & $39.51 \%$ & $41.62 \%$ & $43.18 \%$ & $43.00 \%$ & $41.92 \%$ & $40.59 \%$ & $41.80 \%$ \\
staggered HetSQ & $50.05 \%$ & $42.75 \%$ & $43.12 \%$ & $43.94 \%$ & $43.40 \%$ & $42.13 \%$ & $40.70 \%$ & $41.86 \%$ \\
\hline
\end{tabular}

TABLE III

RATE SAVINGS FOR UNIFORM SOURCES WHEN THE ALLOWABLE NORMALIZED DISTORTION IS INCREASED FROM 0.001 TO 0.01

estimation error, a significant rate savings can be realized and that these savings do not appear to diminish as the number of users is increased.

\section{INTERACTIVE EXTREMIZATION}

Comparing with the straightforward scheme in which each user uses a SW code to forward its observations to the CEO to enable it to learn the arg max, we showed in Section IV that it is possible to save some rate by applying graph coloring. However we showed that the maximum possible such savings is small: one can save at most 2 bits for independent and identically distributed sources and the per user saving as the number of users goes to infinity will be 0 . This motivated us to investigate other coding strategies capable of delivering a larger reduction in rate. While the previous section considered strategies that enabled this rate reduction by relaxing the requirement that the CEO compute the extremizations perfectly to computing them in a lossy manner, here we will revert to the requirement that the extremizations are computed losslessly and focus instead on rate savings obtainable through interactive communication.

Interactive communication is defined to be a method of communication that allows message passing forward and backward multiple times between two or more terminals [38]. It has been shown that interactive communication can provide substantial rate savings over non-interactive communication in some distributed function computation problems [14]. Here, we will apply interactive communication to the extremization problems, and show that a large 
reduction in rate is possible relative to the non-interactive lossless limits presented in Section IV While we will not discuss any fundamental limits as they are not yet available in the literature for the interactive CEO problems under investigation, we will demonstrate that through interaction we can obtain substantial rate savings.

Inspired by the selective multiuser diversity (SMUD) [40] scheme as well as the multi-predefined thresholds [41] scheme which is an extension of SMUD, we propose here the Multi-Thresholds Interactive Scheme (MTIS) between the CEO and the users that efficiently encodes the feedback necessary for the lossless computation of the extremization problems. We show that the MTIS achieves a large reduction in the rate when interaction is utilized when compared with the rate results of Theorem 1 in Section IV] in which each user sends its own message to the CEO by graph coloring.

Here we will model the observations of the users as identically distributed discrete random variables with support set $\mathcal{X}=\left\{\alpha_{1}, \ldots, \alpha_{L}\right\}$ s.t. $0<\alpha_{1}<\alpha_{2}<\ldots<\alpha_{L}$, and cumulative distribution function $F_{x}(x)$. The users each initially occupy a fraction of a bandwidth to communicate to the CEO. The CEO knows the user index and the part of the bandwidth that it corresponds to at the beginning. The interactive communication will occur over multiple rounds indexed by $t$. During each round, only a subset of the users called the online users will participate in the communication, and the CEO will know which users are offline by the information it exchanges with the online users. For instance, in the arg max case, a user remains online only while it is still possible to be the $\arg \max$ based on the information it has received up until this round, and is offline otherwise. The part of communication bandwidth associated with offline users is freed up for use by other communications and is thus not wasted. During round $t$, given the $\operatorname{CDF} F_{t}(x)$, the support set $\mathcal{X}_{t}=\left\{\alpha_{1}^{t}, \ldots, \alpha_{L(t)}^{t}\right\}$ and the $N_{t}$ conditioned on the information that the CEO obtained about the online users thus far, it will determine and send a common message $V_{t}$ to declare a threshold to each of the online users, and each online user $i$ responds with a message $U_{t}^{i}$ to let the CEO know whether or not it is above this threshold for all $i \in\left[N_{t}\right]$. The user will stay online for the next round if it feeds back a 1. Alternatively, if a user feeds back a 0 , but the next threshold $\lambda_{t+1}$ is lower than $\lambda_{t}$ (which indicating that all users replied 0 at round $t$ ), it will also stay online, otherwise this user becomes offline. After receiving all of the feedback bits, the CEO can obtain the information $F_{t+1}(x), \mathcal{X}_{t+1}$ and $N_{t+1}$ for next round's communication. If there is only one user above the threshold $\lambda_{T}$ at the round $T$, this user is the $\arg \max$ and the communication process stops. Similarly, if $\left|\mathcal{X}_{T}\right|=1$, then all of the online users in the next round attain the max, and the communication process stops since the $\mathrm{CEO}$ can pick any one of these users to be the arg max. If more than one online user replies a 1 , then conditioned on all the information received thus far, the new channel distribution parameters for the next round are

$$
\begin{aligned}
& N_{t+1}=\sum_{i=1}^{N_{t}} \mathbb{1}_{x_{i} \geq \lambda_{t}} \\
& \alpha_{1}^{t}=\lambda_{t} \\
& \alpha_{L(t+1)}^{t}=\alpha_{L(t)}^{t} \\
& F_{t+1}(x)=\frac{F_{t}(x)-F_{t}\left(\lambda_{t}\right)}{F_{t}\left(\alpha_{L(t)}^{t}\right)-F_{t}\left(\lambda_{t}\right)}
\end{aligned}
$$


While if all users reply 0, then conditioned on all the information received thus far at the CEO, the new channel distribution parameters for the next round are

$$
\begin{aligned}
& N_{t+1}=N_{t} \\
& \alpha_{1}^{t+1}=\alpha_{1}^{t} \\
& \alpha_{L(t+1)}^{t+1}=\lambda_{t} \\
& F_{t+1}(x)=\frac{F_{t}(x)-F_{t}\left(\alpha_{1}^{t}\right)}{F_{t}\left(\lambda_{t}\right)-F_{t}\left(\alpha_{1}^{t}\right)}
\end{aligned}
$$

The threshold for next round can be generated based on the new information. Hence the algorithm of MTIS operates as follows.

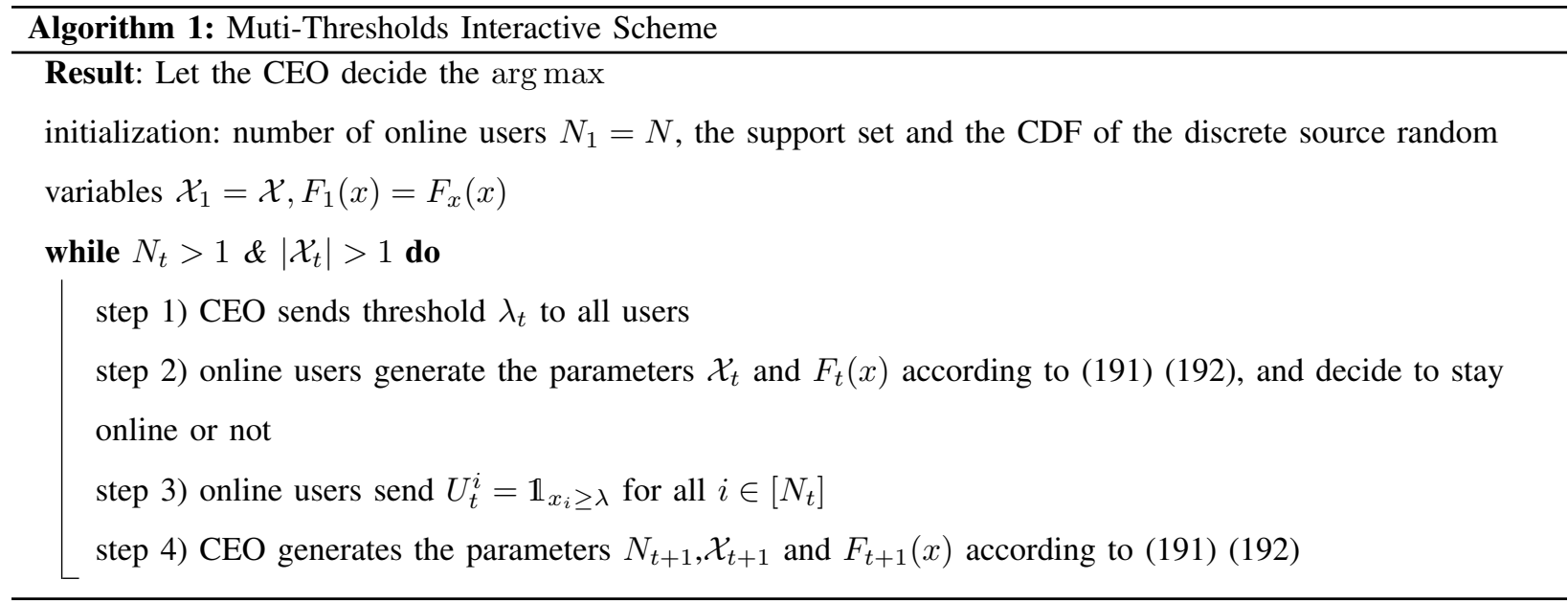

\section{A. Analysis}

Our aim in this subsection is to determine the optimal choice of the thresholds in the interactive scheme in the sense of minimizing the average total amount of rates must incur.

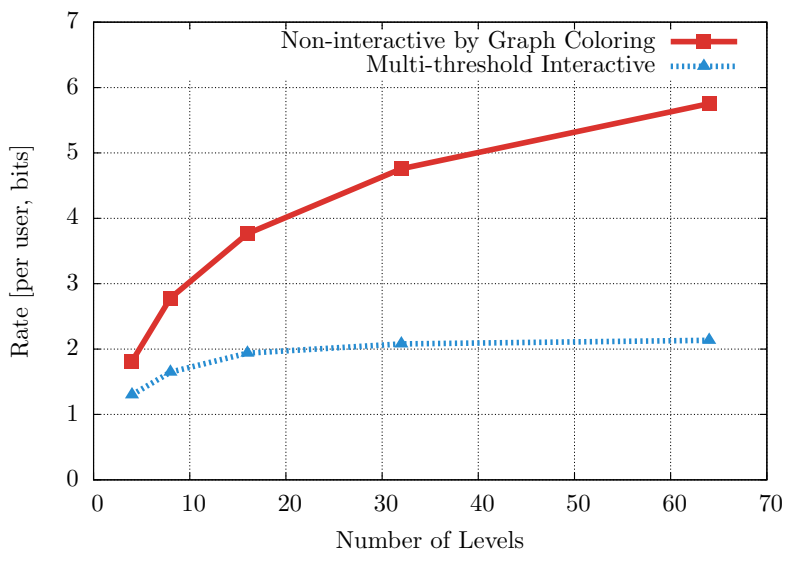

(a)

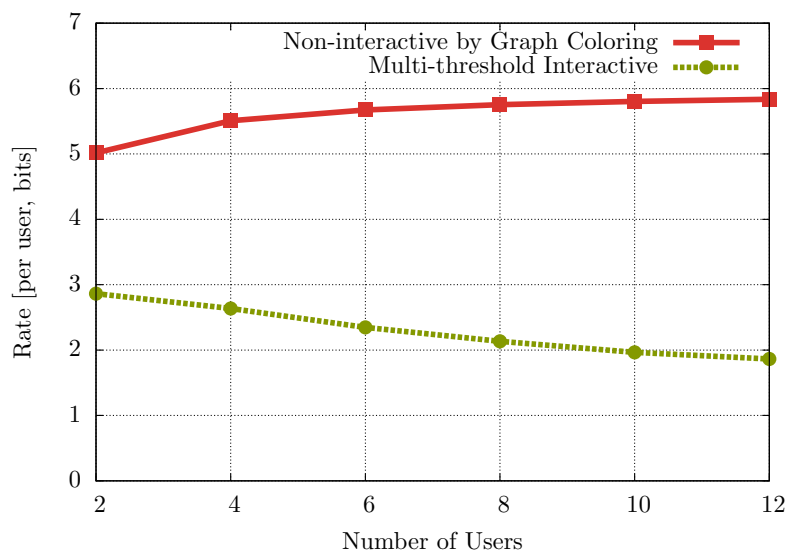

(b)

Fig. 14. MTIS Vs. non interactive for the arg max problem with (a) $N_{1}=8$, and; (b) $\left|\mathcal{X}_{1}\right|=64$ 
Define $R$ to be the total expected number of overhead bits exchanged when using the series of threshold levels $\lambda_{1}, \lambda_{2}, \cdots$, and define $R^{*}$ to be

$$
R^{*}=\min _{\lambda_{1}, \lambda_{2}, \ldots} R\left(\lambda_{1}, \lambda_{2}, \ldots\right)
$$

It is clear that $R^{*}$ will be a function of the initial number of users $N_{1}$ (all of whom are initially online) and $\mathcal{X}_{1}$. We will need the following theorem to solve the optimization problem.

Theorem 16. Problem (193) is a dynamic programming problem.

Proof: We first show there will be a finite stop T for (193). The threshold $\lambda_{t}$ is picking from the support set of the sources $\mathcal{X}=\left\{\alpha_{1}^{t}, \ldots, \alpha_{L(t)}^{t}\right\}$. After each round of communication, the support set will be updated to either $\left\{\alpha_{1}^{t}, \ldots, \lambda_{t}\right\}$ or $\left\{\lambda_{t}, \ldots, \alpha_{L(t)}^{t}\right\}$, hence the size of the support set is monotone decreasing. Therefore finite rounds are needed to decrease the support set to be 1 and the communication stops.

Also, we observe that if policy $\lambda_{1}^{*}, \cdots, \lambda_{T}^{*}$ is the optimal choice of thresholds for initial condition $N_{1},\left\{\alpha_{1}^{1}, \ldots, \alpha_{L(1)}^{1}\right\}$ and $F_{1}(x)$ then the truncated policy $\lambda_{t}^{*}, \cdots, \lambda_{T}^{*}$ will be the optimal choice of thresholds for initial condition $N_{t}$, $\left\{\alpha_{1}^{t}, \ldots, \alpha_{L(t)}^{t}\right\}$ and $F_{t}(x)$, and thus the problem has the form of a dynamic programming problem.

In order to solve this problem, we begin with a one round analysis in which we assume to pick $\lambda_{t}$ as the threshold for round $\mathrm{t}$ and that the thresholds after round $\mathrm{t}$ have been optimized already. Define $R_{t}\left(\lambda_{t}\right)$ as the expected aggregate rate from round $t$ to the end, then

$$
R_{t}\left(\lambda_{t}\right)=H\left(\lambda_{t} \mid \lambda_{1}, \mathcal{X}_{1}, N_{1}, \cdots, \lambda_{t-1}, \mathcal{X}_{t-1}, N_{t-1}\right)+N_{t}+\mathbb{E}\left[R_{t+1}\right]
$$

where the first term represents the minimum number of bits needed to let the users know the threshold in round $t$, the second term represents the total number of bits of feedback from the $N_{t}$ users, and the last term represents the expected rate cost for future rounds which can be further expressed as

$\mathbb{E}\left[R_{t+1}\right]=\sum_{i=0}^{N_{t}} p_{i} \mathbb{E}\left[R_{t+1} \mid i\right]=\left(F_{t}\left(\lambda_{t}\right)\right)^{N_{t}} R^{*}\left(N_{t}, \alpha_{1}^{t}, \lambda_{t}\right)+\sum_{i=1}^{N_{t}}\left(1-F_{t}\left(\lambda_{t}\right)\right)^{i} F_{t}\left(\lambda_{t}\right)^{N_{t}-i} \frac{N_{t} !}{i !\left(N_{t}-i\right) !} R^{*}\left(i, \lambda_{t}, \alpha_{L(t)}^{t}\right)$

where $p_{i}$ represents the probability of $\mathrm{i}$ users reply 1 at round $\mathrm{t}$. The optimal choice of threshold at round $t$ then must satisfy

$$
\lambda_{t}^{*}=\underset{\lambda_{t}}{\arg \min } R_{t}\left(\lambda_{t}\right)
$$

(194) (195) and 196 together form a policy iteration algorithm [46] for this dynamic programming problem.

\section{B. Thresholds vs. Number of Users}

Let us now consider several possible methods of encoding the threshold, and hence several possible values for the quantity $H\left(\lambda_{t} \mid \lambda_{1}, \mathcal{X}_{1}, N_{1}, \cdots, \lambda_{t-1}, \mathcal{X}_{t-1}, N_{t-1}\right)$ in 194. Based on SW codes, the minimum information the CEO needs to broadcast should be the conditional entropy of the threshold given all previous knowledges that the online users have. 
For the purposes of comparison, and ease of the associated algorithm encoder design, let us also consider two additional coding strategies which are easy to implement. We will see that these two strategies also require less communication than the non-interaction scheme. The first strategy is to encode the threshold with no conditioning

$$
U_{t}=H\left(\lambda_{t}\right)=\log _{2}\left|\mathcal{X}_{t}\right|
$$

Motivated by the idea that the users may calculate the optimal choice of threshold themselves rather than receiving it, we provide the second strategy that the BS broadcasts the number of currently online users. Observe that the optimal policy $\lambda^{*}$ at each round is determined by the information the CEO has, including $N_{t}, f_{t}(x)$ and $\mathcal{X}_{t}=\left\{\alpha_{1}^{t}, \ldots, \alpha_{L(t)}^{t}\right\}$. We show that it is enough to let the users calculate the threshold by broadcasting $N_{t}$ by induction.

Theorem 17. The number of online users $N_{t}$ is a sufficient statistic of the optimal threshold $\lambda_{t}^{*}$.

Proof: 194 195 196 show that the CEO determines the $\lambda_{t}^{*}$ by the information of $\left\{\left(F_{i}(x), \mathcal{X}_{i}, N_{i}\right): i \in[t]\right\}$, hence it suffices to show that the users can learn $F_{t}(x)$ and $\mathcal{X}_{t}$ by knowing $N_{t}$ at round $t$. We prove it by induction. At round 1 , each user has the $\operatorname{CDF} F_{1}(x)$, the support set $\mathcal{X}_{1}=\left\{\alpha_{1}^{1}, \ldots, \alpha_{L(1)}^{1}\right\}$ and its own value $x_{i}$, hence the optimal threshold $\lambda_{1}^{*}$ can be calculated after receiving the initial number of the online users $N_{1}$. Suppose that at round $t-1$ the users successfully compute the threshold $\lambda_{t-1}^{*}$ by the information $N_{t-1}, F_{t-1}(x)$ and $\mathcal{X}_{t-1}=\left\{\alpha_{1}^{t-1}, \ldots, \alpha_{L(t-1)}^{t-1}\right\}$. Now at round $t$ for any user $i \in\left[N_{t-1}\right]$, if it receives $N_{t}=N_{t-1}$ and its value is below the threshold $\lambda_{t-1}$ which means it replied a 0 at previous round, then it knows that every user must be below the previous threshold and $\mathcal{X}_{t}=\left\{\alpha_{1}^{t-1}, \lambda_{t-1}^{*}\right\}$; similarly if it receives $N_{t}=N_{t-1}$ and its value is above the threshold $\lambda_{t-1}$, then it knows that every user must be above the previous threshold and $\mathcal{X}_{t}=\left\{\lambda_{t-1}^{*}, \alpha_{L(t-1)}^{t-1}\right\}$. Therefore the $\mathcal{X}_{t}$ can be renewed at each user by the following rules

$$
\mathcal{X}_{t}= \begin{cases}\left\{\lambda_{t-1}^{*}, \alpha_{L(t-1)}^{t-1}\right\} & \text { if } N_{t}<N_{t-1} \\ \left\{\alpha_{1}^{t-1}, \lambda_{t-1}^{*}\right\} & \text { if } N_{t}=N_{t-1} \text { and } \lambda_{t-1}^{*}>x_{i} . \\ \left\{\lambda_{t-1}^{*}, \alpha_{L(t-1)}^{t-1}\right\} & \text { if } N_{t}=N_{t-1} \text { and } \lambda_{t-1}^{*} \leq x_{i}\end{cases}
$$

Note that the user will turn offline if $N_{t}<N_{t-1}$ and $\lambda_{t-1}^{*}>x_{i}$ and stay online otherwise. The updated CDF $F_{t}(x)$ can be get by 191 192 once $\mathcal{X}_{t}$ has been renewed. Therefore, the threshold $\lambda_{t}^{*}$ can be determined after each user receiving the $N_{t}$.

\section{Results-Interaction in the arg max case}

Having identified the policy iteration form of the problem of minimizing the expected aggregate rate exchanged for the MTIS scheme for determining the user with the arg max, we now solve the policy iteration for the various methods of communicating the thresholds. We will measure the amount of communication required in each case and compare with the amount of information which must be transmitted without interaction. As we mentioned 


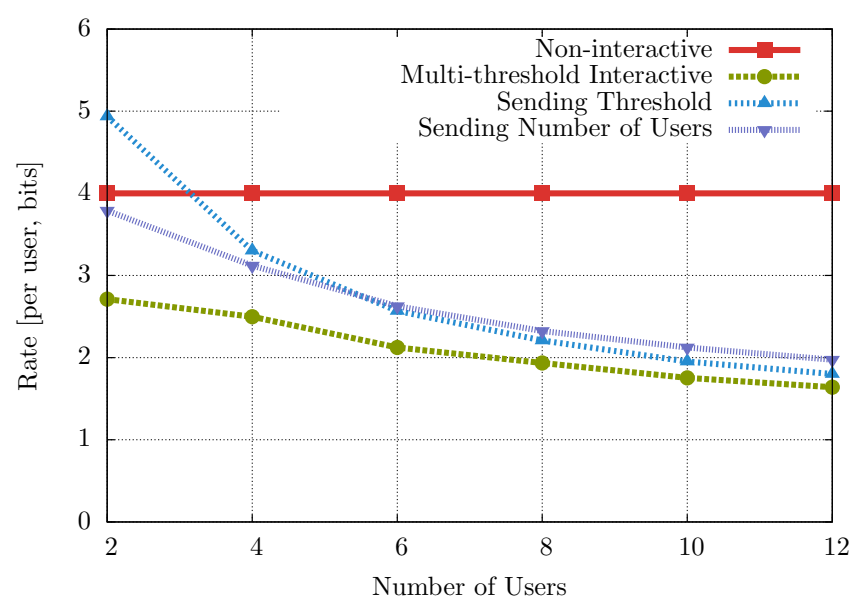

Fig. 15. A comparison of sending thresholds and sending number of users with $\left|\mathcal{X}_{1}\right|=16$.

before, 194 195 can be solved by iteration with the boundary condition

$$
R^{*}\left(N_{t}, \mathcal{X}_{t}\right)=0
$$

if $N_{t}=1$ or $\left|\mathcal{X}_{t}\right|=1$. Fig. 14a, 14b, 18a, 18b, 15, 16a and $16 \mathrm{~b}$ present the number of bits communicated under the various schemes when the sources are uniformly distributed. Fig. 14a compares the bits communicated by MTIS, with SW coded thresholds achieving the conditional entropies (194), and the non-interactive scheme with $N_{1}=8$, while Fig. $14 \mathrm{~b}$ performs the same comparison with $\mathcal{X}_{1}=64$. From both figures we can see significant rate savings through interaction when calculating the arg max.

As mentioned in previous subsection, we suggested two simple encoding strategies for the base station to broadcast which include Huffman encoding the $\lambda_{t}^{*}$ with no conditioning on previous thresholds and Huffman encoding the $N_{t}$. Fig. 15 shows the number of bits that must be exchanged when these methods are used. The strategy of sending the threshold outperforms the strategy of sending the number of users in the situation that the initial number $N_{1}$ is large; while when $N_{1}$ is small, the latter shows better performance. The minimum between these two schemes requires an amount of communication close to the best scheme, which SW encodes the thresholds.

\section{Results-max and (arg max, max) Case}

We can also apply the achievable interaction scheme in the problem that the exact maximum value need to be decided as well as the problem that both the max and arg max need to be decided, following the same analysis as (191) to 196 with the only difference being the boundary conditions. Instead of [199], we will have the following condition for determining the max and the pair

$$
R^{*}\left(N_{t}, \mathcal{X}_{t}\right)=0 \Longleftrightarrow\left|\mathcal{X}_{t}\right|=1
$$

For the problem that the CEO wants to learn the max or the pair (max, arg max), Fig. 16a compares the bits communicated by MTIS, with SW coded thresholds achieving the conditional entropies (194), and the non- 


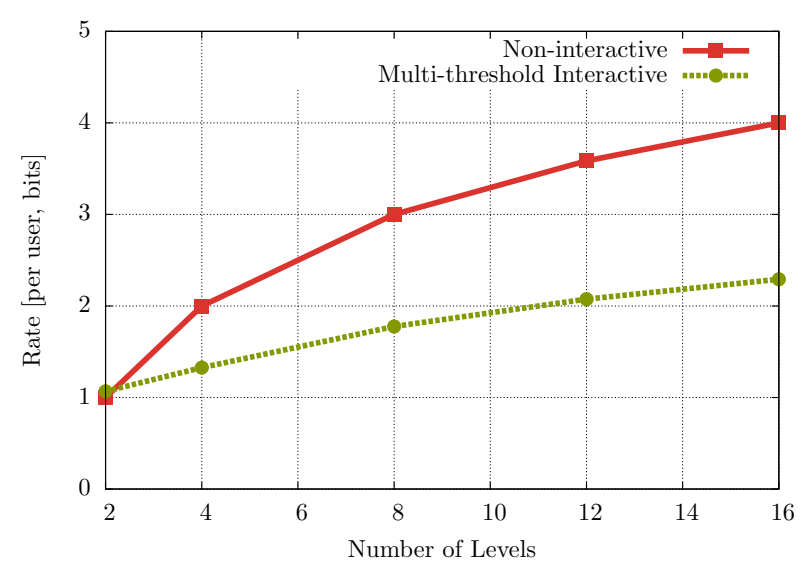

(a)

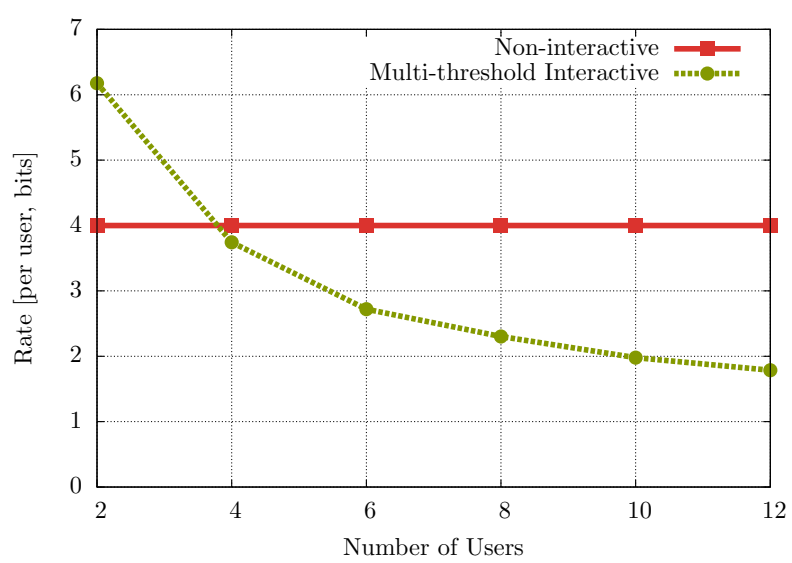

(b)

Fig. 16. MTIS Vs. non-interactive for the max problem with (a) $N_{1}=8$, and; (b) $\left|\mathcal{X}_{1}\right|=16$

interactive scheme with $N_{1}=8$, while Fig. $16 b$ performs the same comparison with $\mathcal{X}_{1}=16$. Note that case 1 and case 3 share the same boundary conditions and hence have the same rates because once the CEO knows the maximum value, it can pick any one of the online users that achieves the maximum. Also note that by Theorem 4 the one-way fundamental limit of determining the $\max$ is $N H(X)$ because we have selected $\min \mathcal{X}>0$.

\section{E. Scaling Laws}

We have shown for the lossless non-interactive communication, one can have at most 2 bits saving for the $\arg \max$ case, and the per user saving goes to 0 as the number of users goes to infinity. Now we will see our proposed interactive scheme will exhibit a better scaling law.

Theorem 18. For the case that two users each observe uniformly distributed independent discrete sources, the aggregate expected rate required to losslessly determine the arg max by interactive communication satisfies

$$
R^{*}<6-6\left(\frac{1}{2}\right)^{\left\lceil\log _{2} L\right\rceil}<6
$$

hence the per-user rate goes to 0 as $N$ goes to infinity.

Proof: We will derive an upper bound on the amount of information exchanged by MTIS by choosing nonoptimal thresholds and transmitting $N_{t}$ instead of the threshold. The users, instead of computing $\lambda_{t}^{*}$ by dynamic programming, will always pick the median of $\mathcal{X}_{t}$ as the threshold and send a 1 bit message indicating whether its observation is in $\left\{\alpha_{1}^{t}, \lambda_{t-1}\right\}$ or $\left\{\lambda_{t}, \ldots, \alpha_{L(t)}^{t}\right\}$. The CEO then also replies a 1 bit message indicating whether or not the two users are in the same region. The communication process stops if the two users are not in the same region, otherwise the problem degenerates to a 2-user arg max problem with support set shrinking to a half of the original size. Define $R(L)$ as the expected aggregate rate by this interactive scheme with support set $\left\{\alpha_{1}, \ldots, \alpha_{L}\right\}$ 
in the 2-user arg-max problem.

$$
\begin{aligned}
R(L) & \stackrel{(a)}{=} 2+1+\left(p_{1}^{t} p_{2}^{t}\right)(R(\lceil L / 2\rceil)+R(\lfloor L / 2\rfloor)) \\
& \leq 3+2 p_{1}^{t} p_{2}^{t} R(\lceil L / 2\rceil) \\
& \leq 3+0.5(R(\lceil L / 2\rceil))
\end{aligned}
$$

where $p_{1}^{t}=\mathbb{P}\left(x \in\left\{\mathcal{X}_{1}^{t}, \lambda_{t-1}\right\}\right), p_{2}^{t}=\mathbb{P}\left(x \in\left\{\lambda_{t-1}, \mathcal{X}_{L(t)}^{t}\right\}\right)$. Where the 2 in 202 stands for the 2 bits communicated by the two users in this round, the 1 stands for the replied bit from the CEO, and the last term stands for the case that both users either reply 1 or 0 . As 199 suggests, we have $R(1)=0$, hence for any $\mathcal{X}=\left\{\alpha_{1}, \ldots, \alpha_{L}\right\}$ we have

$$
\begin{aligned}
R(L)-6 & \leq \frac{1}{2}((R(\lceil L / 2\rceil))-6) \\
& \leq\left(\frac{1}{2}\right)^{m}(R(1)-6) \\
& =-6\left(\frac{1}{2}\right)^{m}
\end{aligned}
$$

where $2^{m-1} \leq L \leq 2^{m}$, and therefore

$$
R^{*} \leq R(L) \leq 6-6\left(\frac{1}{2}\right)^{m}<6 .
$$

Theorem 19. Let $\Delta_{A}=R_{A}^{*}-R^{*}$ be the rate saving of the proposed interactive scheme w.r.t. the lossless noninteractive limit $R_{A}^{*}$ in the arg max problem, the per-user saving $\Delta_{A} / N$ satisfies

$$
\lim _{N \rightarrow \infty} \frac{\Delta_{A}}{N} \geq H(X)-1
$$

Proof: We propose an interactive scheme which will derive an upper bound on the amount of information exchanged by MTIS by choosing $\lambda=\max \mathcal{X}$. Define $R_{U}(\mathcal{X}, N)$ as the expected aggregate rate of this scheme, we know $R_{U} \geq R^{*}$, and

$$
\begin{aligned}
R_{U}\left(\left\{\alpha_{1}, \ldots, \alpha_{L}\right\}, N\right) & =\left(1-p_{L}\right)^{N} R_{U}\left(\left\{\alpha_{1}, \ldots, \alpha_{L-1}\right\}, N\right)+\left(1-\left(1-p_{L}\right)^{N}\right) \cdot 0+H(X)+N \\
& \leq\left(1-p_{L}\right)^{N} R_{U}\left(\left\{\alpha_{1}, \ldots, \alpha_{L}\right\}, N\right)+H(X)+N \\
& \leq\left(1-p_{L}\right)^{N} N H(X)+H(X)+N
\end{aligned}
$$

where the first two terms in 206 stand for the expected rate cost for future rounds, $p_{L}=\mathbb{P}\left(X=\alpha_{L}\right), H(X)$ stands for the bits required to send the threshold $\lambda=\max \mathcal{X}$ and $N$ stands for the bits replied by the $N$ users. Hence by 28, 206, and the fact that $\lim _{N \rightarrow \infty}\left(1-p_{L}\right)^{N}=0$, we have

$$
\begin{aligned}
\lim _{N \rightarrow \infty} \frac{\Delta_{A}}{N} & \geq \lim _{N \rightarrow \infty} \frac{1}{N}\left((N-2) H(X)-\sum_{i=1}^{L-1} p_{i, i+1} \log _{2} p_{i, i+1}-p_{1} \log _{2} p_{1}-p_{L} \log _{2} p_{L}-\left(1-p_{L}\right)^{N} \cdot N H(X)-H(X)-N\right) \\
& =H(X)-1 .
\end{aligned}
$$




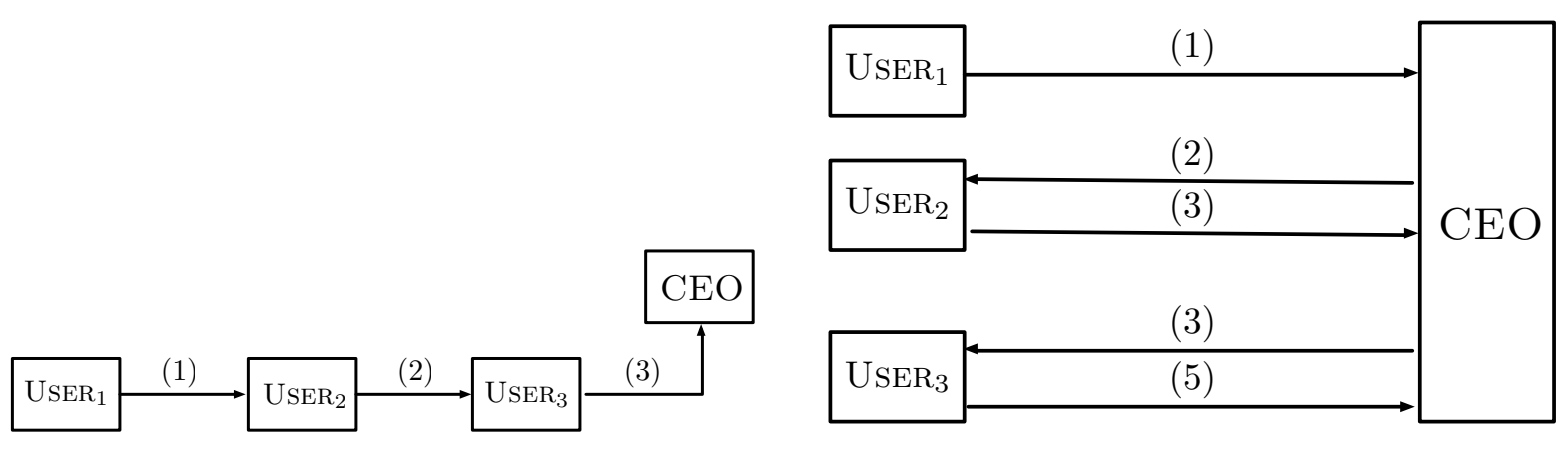

(a)

(b)

Fig. 17. The 3 users case of the CEO extremization problem with (a) RIS, and; (b) NBIS

\section{F. Compare with Other Interactive Schemes}

As an interesting point of comparison, we compare the MTIS with another two interactive schemes. Both of the two schemes are given in [14] as examples that show interaction can enable rate savings relative to noninteractive schemes in distributed function computation problems. In both schemes, it is assumed that when the user sends an message, the CEO knows without cost which user this message is from. Additionally, in the first scheme, referred to as Relay Interactive Scheme (RIS), the users transmit sequentially with one user transmitting at a time for reception by the next user. The second scheme, called Non-Broadcasting Interactive Scheme (NBIS), has an additional constraint that all communication must occur between the CEO and users and the CEO can only communicate to one user at a time. Here we illustrate the schemes for 3 users. Pseudocode for the two schemes is provided in Algorithms 2 and 3 respectively.

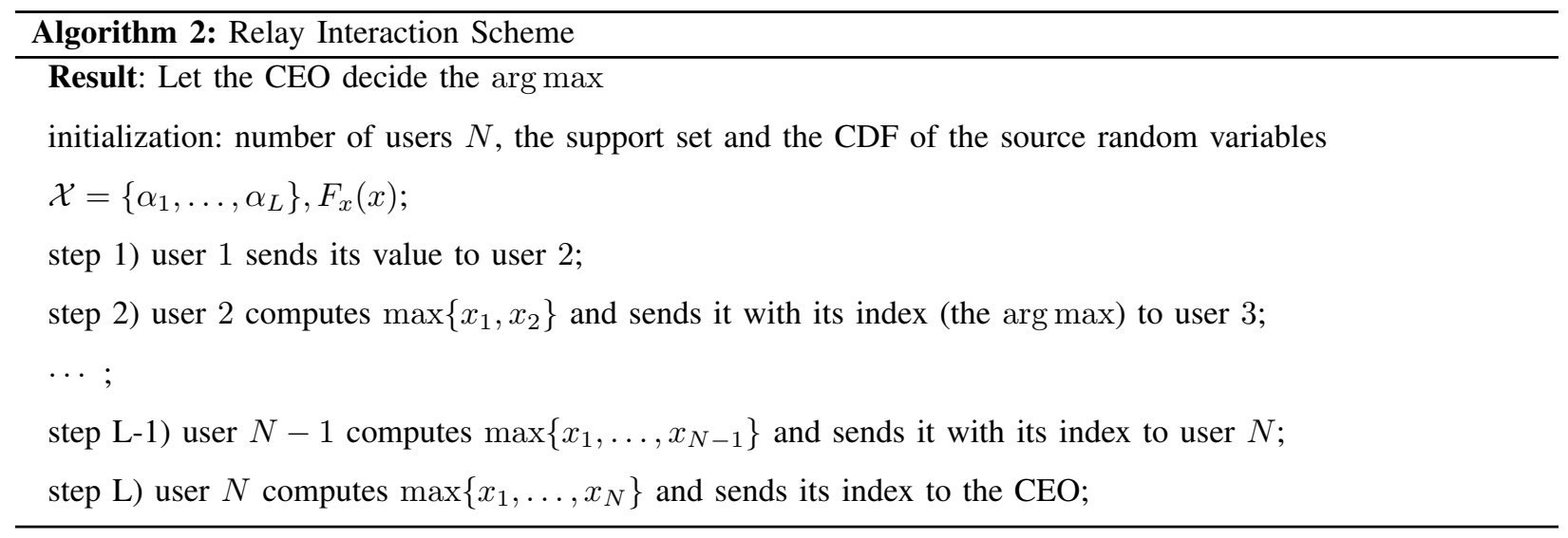




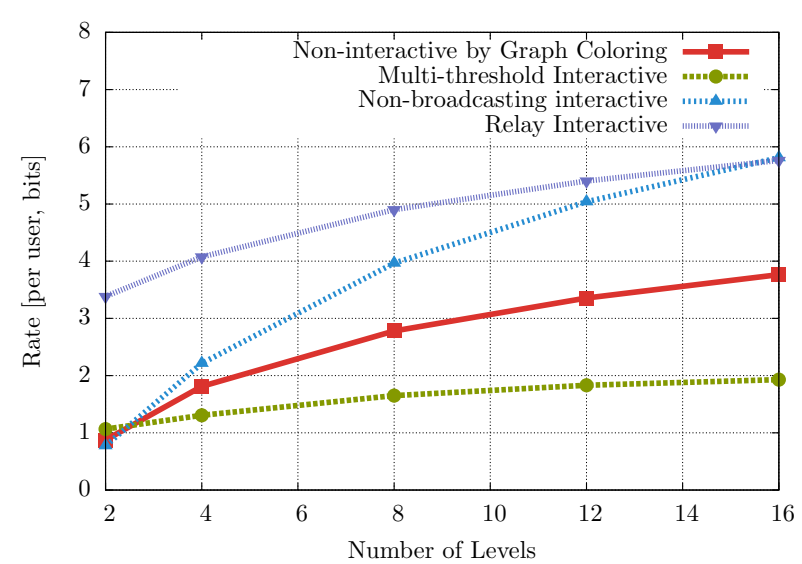

(a)

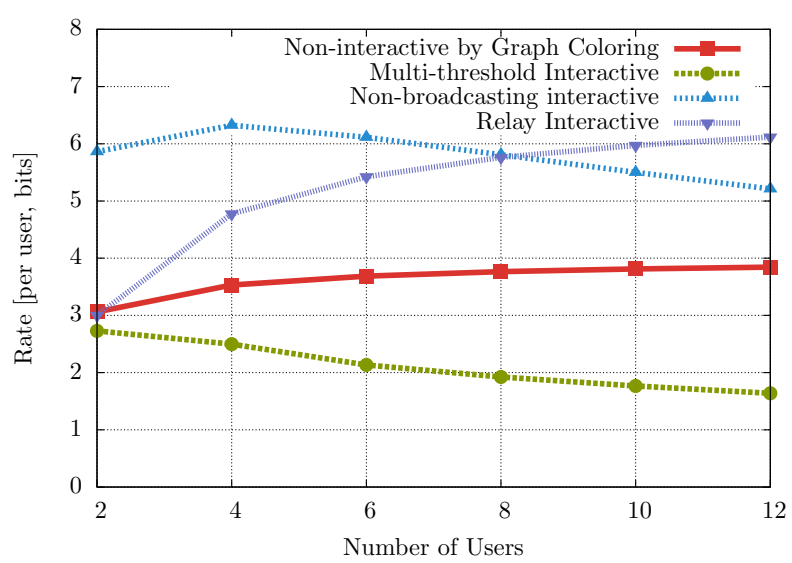

(b)

Fig. 18. A comparison of the three interactive schemes with (a) $N_{1}=8$, and; (b) $\left|\mathcal{X}_{1}\right|=16$

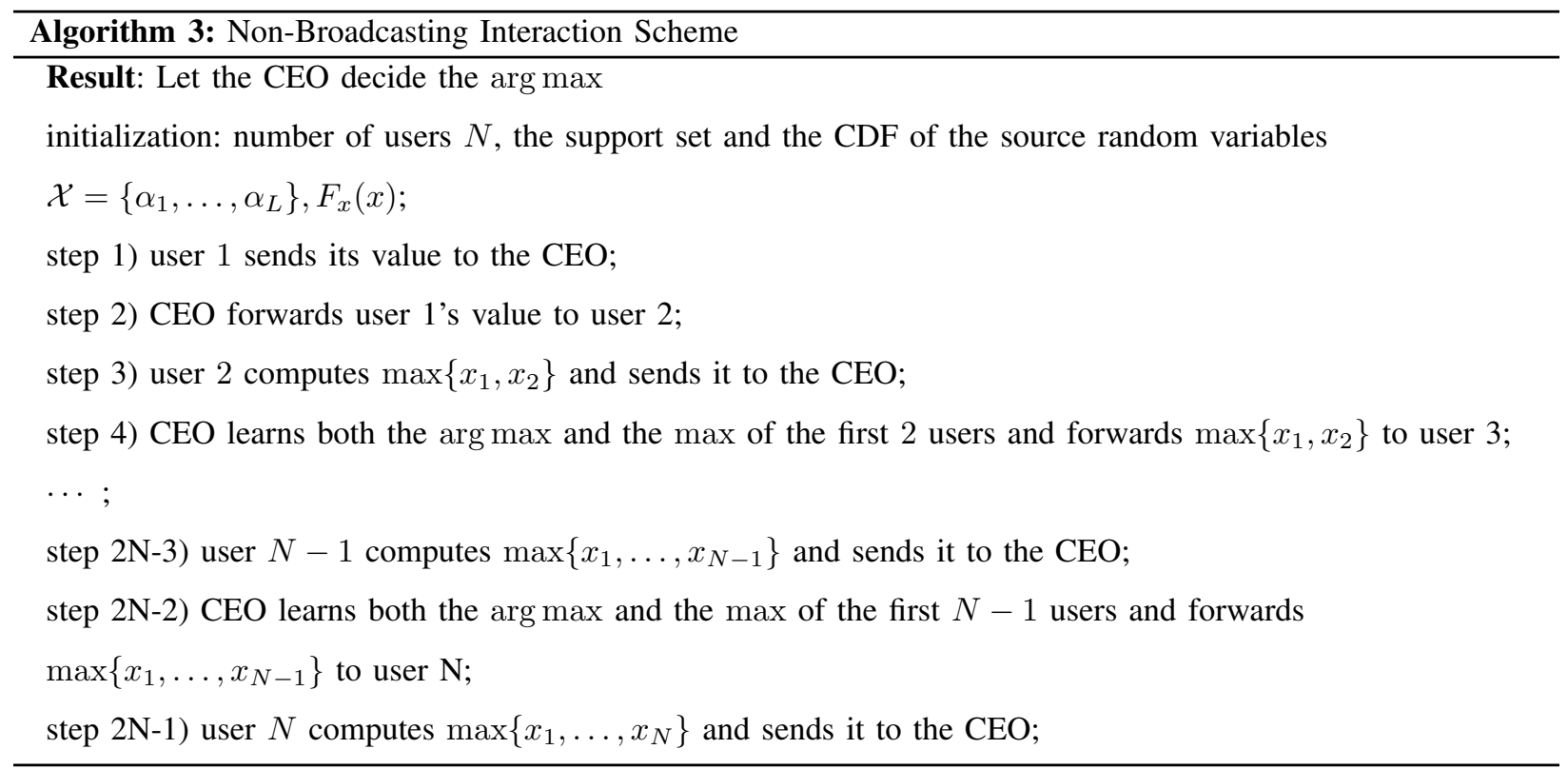

In Fig. 18a and Fig. 18b, we see that for uniformly distributed sources, the NBIS has better performance than MTIS when there is only two users. For most of the cases, the MTIS utilizes fewer overhead bits than the other two schemes.

In summary, we observe from Fig. 14a - Fig. 18b that the MTIS provides a substantial saving in sum rate relative to the the non-interactive scheme as well as the RIS and the NBIS while still obtaining the answer losslessly. In fact, we observe from Theorem 19 that the per-user rate goes to 1 as the number of users goes to infinity, which is a very large reduction relative to the minimum necessary communication if non-interaction is required. 


\section{CONCLUSION}

In this paper, we considered resource allocation problems in which a resource controller needs to compute an extremization function (one or both of the functions max, $\arg \max$ ) over a series of $N$ remote users. Designs were developed that minimized the amount of information exchange necessary for this remote function computation. We first showed that, in most of the cases where the extremization must be computed losslessly, at most two bits can be saved relative to the direct scheme in which the users simply forward their metrics to the controller which computes the function. In contrast to this lossless case, we observed that substantial rate savings can be achieved if the controller tolerates even a small amount of distortion in computing the function. In particular, we developed simple quantizers for remote extremization whose rate distortion performance closely matches the optimal rate distortion curve. Alternatively, if no distortion can be tolerated, we demonstrate that substantial rate savings can still be achieved if the controller and the users are allowed to interactively communicate. An attractive feature of both the interactive and lossy paradigms for remote extremization is that the rate saving obtained improve with the number of users. An important direction for future work is to further reduce the rate necessary via lossy interactive computation, by building a hybrid combining the developed lossy and interactive schemes.

\section{REFERENCES}

[1] B. D. Boyle, J. M. Walsh, and S. Weber, "Distributed scalar quantizers for subband allocation," in Conf. Information Sciences and Systems (CISS), March 2014.

[2] J. Ren and J. M. Walsh, "Interactive communication for resource allocation," in Conf. Information Sciences and Systems (CISS), March 2014.

[3] S. Verdu and S. Shamai, "Variable-rate channel capacity," IEEE Trans. Inf. Theory, vol. 56, no. 6, pp. 2651-2667, Jun. 2010.

[4] M. Luby, "Lt codes," in Proceedings of the 43rd Symposium on Foundations of Computer Science, 2002, pp. 271-280.

[5] U. Erez, M. D. Trott, and G. W. Wornell, "Rateless coding for Gaussian channels," IEEE Trans. Inf. Theory, vol. 58, no. 2, pp. 530-547, Feb. 2012.

[6] E-UTRA; Physical Channels and Modulation (Release 8), 3GPP Technical Specification TS36211-890, Dec. 2009.

[7] D. Katabi and J. Wroclawski, “A framework for scalable global ip-anycast (GIA),” SIGCOMM Comput. Commun. Rev., vol. 30, no. 4, pp. $3-15,2000$.

[8] J. G. Proakis and M. Salehi, Communication Systems Engineering. Prentice Hall, 1994.

[9] H. R. Varian, Microeconomic Analysis. W.M. Norton, 1992.

[10] J. Rawls, A Theory of Justics. Harvard University Press, 1971.

[11] V. Krishna, Auction Theory, 2nd ed. Academic Press, 2010.

[12] I. Butun, S. D. Morgera, and R. Sankar, "A survey of intrusion detection systems in wireless sensor networks," IEEE Commun. Surveys Tuts., vol. 16, no. 1, pp. 266-282, 2014.

[13] D. Slepian and J. K. Wolf, "Noiseless coding of correlated information sources," IEEE Trans. Inf. Theory, vol. 19, no. 4, pp. 471-480, Jul. 1973.

[14] N. Ma and P. Ishwar, "Some results on distributed source coding for interactive function computation," IEEE Trans. Inf. Theory, vol. 57, no. 9, pp. 6180-6195, Sep. 2011.

[15] H. S. Witsenhausen, "The zero-error side information problem and chromatic numbers," IEEE Trans. Inf. Theory, vol. 22, no. 5, pp. 592-593, Sep. 1976.

[16] A. Orlitsky and J. R. Roche, "Coding for computing," IEEE Trans. Inf. Theory, vol. 47, no. 3, pp. 903-917, Mar. 2001.

[17] M. Sefidgaran and A. Tchamkerten, "Distributed function computation over a rooted directed tree," submitted to IEEE Trans. Inf. Theory. [Online]. Available: http://arxiv.org/pdf/1312.3631v1.pdf 
[18] V. Doshi, D. Shah, M. Medard, and S. Jaggi, "Functional compression through graph coloring," IEEE Trans. Inf. Theory, vol. 56, no. 8, pp. 3901-3917, Aug. 2010.

[19] T. Berger, Z. Zhang, and H. Viswanathan, "The CEO problem [multiterminal source coding]," IEEE Trans. Inf. Theory, vol. 42, no. 3, pp. 887-902, May 1996.

[20] C. E. Shannon, "Coding theorems for a discrete source with a fidelity criterion," IRE Nat. Conv. Rec., vol. 7, no. 4, pp. 142-163, 1959.

[21] T. Berger, Rate Distortion Theory: A Mathematical Basis for Data Compression. Englewood Cliffs, N.J.: Prentice-Hall, 1971.

[22] R. G. Gallager, Informattion Theory and Reliable Communication. New York: Wiley, 1968.

[23] T. Berger, "Multiterminal source coding," The Information Theory Approach to Communications, vol. 22, 1977.

[24] S. Tung, "Multiterminal source coding," Ph.D. dissertation, Cornell University, 1978.

[25] S. Arimoto, "An algorithm for computing the capacity of arbitrary discrete memoryless channels," IEEE Trans. Inf. Theory, vol. 18, no. 1, pp. 14-20, 1972.

[26] R. E. Blahut, "Computation of channel capacity and rate-distortion functions," IEEE Trans. Inf. Theory, vol. 18, no. 4, pp. 460-473, 1972.

[27] I. Csiszár, "On the computation of rate-distortion functions (corresp.)," IEEE Trans. Inf. Theory, vol. 20, no. 1, pp. 122-124, 1974.

[28] P. Boukris, "An upper bound on the speed of convergence of the Blahut algorithm for computing rate-distortion functions (corresp.)," IEEE Trans. Inf. Theory, vol. 19, no. 5, pp. 708-709, 1973.

[29] Y. Oohama, "Rate-distortion theory for Gaussian multiterminal source coding system with several side informations at the decoder," IEEE Trans. Inf. Theory, vol. 51, no. 7, pp. 2577-2593, Jul. 2005.

[30] V. Prabhakaran, D. Tse, and K. Ramchandran, "Rate region of the quadratic Gaussian CEO problem," in Proc. Int. Symp. Inform. Theory (ISIT), Jun. 2004, p. 117.

[31] A. B. Wagner and V. Anantharam, "An improved outer bound for multiterminal source coding," IEEE Trans. Inf. Theory, vol. 54, no. 5, pp. 1919-1937, May 2008.

[32] A. D. Wyner and J. Ziv, "The rate-distortion function for source coding with side information at the decoder," IEEE Trans. Inf. Theory, vol. IT-22, no. 1, pp. 1-10, Jan. 1976.

[33] G. Ku, J. Ren, and J. M. Walsh, "Computing the rate distortion region for the CEO problem with independent sources," IEEE Trans. Signal Process., 2014, submitted.

[34] V. Misra, V. K. Goyal, and L. R. Varshney, "Distributed scalar quantization for computing: High-resolution analysis and extensions," IEEE Trans. Inf. Theory, vol. 57, no. 8, pp. 5298-5325, 2011.

[35] R. Zamir and T. Berger, "Multiterminal source coding with high resolution," IEEE Trans. Inf. Theory, vol. 45, no. 1, pp. 106-117, 1999.

[36] A. B. Wagner, S. Tavildar, and P. Viswanath, "Rate region of the quadratic Gaussian two-encoder source-coding problem," IEEE Trans. Inf. Theory, vol. 54, no. 5, pp. 1938-1961, 2008.

[37] S. D. Servetto, "Achievable rates for multiterminal source coding with scalar quantizers," in Conf. Rec. of the 39th Asilomar Conf. Signals, Systems and Computers, 2005, pp. 1762-1766.

[38] A. H. Kaspi, "Two-way source coding with a fidelity criterion," IEEE Trans. Inf. Theory, vol. 31, no. 6, pp. 735-740, Nov. 1985.

[39] N. Ma and P. Ishwar, "Interaction strictly improves the wyner-ziv rate-distortion function," in International Symposium on Information Theory, 2010, pp. 61-65.

[40] D. Gesbert and M. S. Alouini, "How much feedback is multi-user diversity really worth?" in Proc. IEEE Int. Conf. Commun., 2004, pp. 234-238.

[41] V. Hassel, M. S. Alouini, D. Gesbert, and G. E. Oien, "Exploiting multiuser diversity using multiple feedback thresholds," in Proc. IEEE Veh. Technol. Conf., 2005, pp. 1302-1306.

[42] V. Doshi, D. Shah, M. Medard, and S. Jaggi, "Graph coloring and conditional graph entropy," in 40th Asilomar Conf. on Signals, Systems, and Computers, 2006, pp. 2137-2141.

[43] J. Ren and J. M. Walsh, "Interactive communication for resource allocation,” Drexel University, Dept. of ECE, Tech. Rep., Dec. 2013.

[44] K. Sayood, Introduction to Data Compression, 4th ed. Elsevier, 2012.

[45] N. Farvardin and J. W. Modestino, "Optimum quantizer performance for a class of non-Gaussian memoryless sources," IEEE Trans. Inf. Theory, vol. 30, no. 3, pp. 485-497, 1984.

[46] D. P. Bertsekas, Dynamic Programming and Optimal Control. Athena Scientific, 2005. 


\section{DISCLAIMER}

The views and conclusions contained herein are those of the authors and should not be interpreted as necessarily representing the official policies or endorsements, either expressed or implied, of the Air Force Research Laboratory or the U.S. Government.

\section{APPENDIX}

\section{A. Proofs}

1) Proof of Theorem 2. Proof: First we prove 1. In user 1's characteristic graph $G_{1}\left(V_{1}, E_{1}\right)$ where $V_{1}=$ $\mathcal{X}=\left\{\alpha_{1}, \ldots, \alpha_{L}\right\}$, by Lemma 2, we must have $\left\{\alpha_{i}, \alpha_{j}\right\} \in G_{1}$ if $|i-j| \geq 2$. Now we consider the pair of vertices $\left\{\alpha_{i}, \alpha_{i+1}\right\}$ for any $i \in\{1, \ldots, L-1\}$. When $\bmod (n, 2) \neq \bmod (i, 2)$, there exists a sequence $\boldsymbol{x}_{\backslash\{1\}}=\left(\alpha_{i}, \ldots, \alpha_{i}\right)$ satisfying $f_{n}^{*}\left(\alpha_{i}, \boldsymbol{x}_{\backslash\{1\}}\right)=f_{n-1}^{*}\left(\boldsymbol{x}_{\backslash\{1\}}\right)+1$ by 26, and $f_{n}^{*}\left(\alpha_{i+1}, \boldsymbol{x} \backslash\{1\}\right)=1$ by 25 . Note that $f_{n}^{*}(\boldsymbol{x})>0$ for all $n$. This implies $f_{n}^{*}\left(\alpha_{i}, \boldsymbol{x}_{\backslash\{1\}}\right)>f_{n}^{*}\left(\alpha_{i+1}, \boldsymbol{x} \backslash\{1\}\right)$, hence $\left\{\alpha_{i}, \alpha_{i+1}\right\} \in G_{1}$. Next we will prove $\left\{\alpha_{i}, \alpha_{i+1}\right\} \notin G_{1}\left(f_{n}^{*}\right)$ if $\bmod (n, 2)=\bmod (i, 2)$. Since $\mathcal{X}^{n-1}=\left\{\boldsymbol{x}_{\backslash\{1\}} \mid \max \{\boldsymbol{x} \backslash\{1\}\}<\right.$ $\left.\alpha_{i+1}\right\} \bigcup\left\{\boldsymbol{x}_{\backslash\{1\}} \mid \max \left\{\boldsymbol{x}_{\backslash\{1\}}\right\}=\alpha_{i+1}\right\} \bigcup\left\{\boldsymbol{x}_{\backslash\{1\}} \mid \max \left\{\boldsymbol{x}_{\backslash\{1\}}\right\}>\alpha_{i+1}\right\}$, it suffices to show that for any given $\boldsymbol{x}_{\backslash\{1\}}$ in these three sets, the function will not differ when $\bmod (n, 2)=\bmod (i, 2)$. For $\boldsymbol{x}_{\backslash\{1\}} \in\left\{\boldsymbol{x}_{\backslash\{1\}} \mid \max \left\{\boldsymbol{x}_{\backslash\{1\}}\right\}<\right.$ $\left.\alpha_{i+1}\right\}$, we observe that $f_{n}^{*}\left(\alpha_{i+1}, \boldsymbol{x}_{\backslash\{1\}}\right)=f_{n}^{*}\left(\alpha_{i}, \boldsymbol{x}_{\backslash\{1\}}\right)=1$ by 25 and 26 . For $\boldsymbol{x}_{\backslash\{1\}} \in\left\{\boldsymbol{x}_{\backslash\{1\}} \mid \max \left\{\boldsymbol{x}_{\backslash\{1\}}\right\}=\right.$ $\left.\alpha_{i+1}\right\}$, we observe that $f_{n}^{*}\left(\alpha_{i+1}, \boldsymbol{x}_{\backslash\{1\}}\right)=f_{n}^{*}\left(\alpha_{i}, \boldsymbol{x}_{\backslash\{1\}}\right)=f_{n-1}^{*}\left(\boldsymbol{x}_{\backslash\{1\}}\right)+1$ by 26, and 27. Finally for $\boldsymbol{x}_{\backslash\{1\}} \in\left\{\boldsymbol{x}_{\backslash\{1\}} \mid \max \left\{\boldsymbol{x}_{\backslash\{1\}}\right\}>\alpha_{i+1}\right\}$, we also observe that $f_{n}^{*}\left(\alpha_{i}, \boldsymbol{x}_{\backslash\{1\}}\right)=f_{n}^{*}\left(\alpha_{i+1}, \boldsymbol{x}_{\backslash\{1\}}\right)=f_{n-1}^{*}\left(\boldsymbol{x}_{\backslash\{1\}}\right)+1$ by 27 . Therefore in user 1 's characteristic graph $G_{1}$, we have

$$
\left\{\alpha_{i}, \alpha_{j}\right\} \notin G_{1} \Longleftrightarrow \bmod (n, 2)=\bmod (i, 2) \& j=i+1
$$

In user 2's characteristic graph $G_{2}\left(V_{2}, E_{2}\right)$ where $V_{2}=\mathcal{X}=\left\{\alpha_{1}, \ldots, \alpha_{L}\right\}$, similarly by Lemma 2 , we must have $\left\{\alpha_{i}, \alpha_{j}\right\} \in G_{2}$ if $|i-j| \geq 2$. Now we consider the pair of vertices $\left\{\alpha_{i}, \alpha_{i+1}\right\}$ for any $i \in\{1, \ldots, L-1\}$. When $\bmod (n, 2)=\bmod (i, 2)$, there exists a sequence $\left(\alpha_{i}, \ldots, \alpha_{i}\right)$ satisfying $f_{n}^{*}\left(\alpha_{i}, \ldots, \alpha_{i}\right)=1$ by 26 , and another sequence $\left(\alpha_{i}, \alpha_{i+1}, \alpha_{i}, \ldots, \alpha_{i}\right)$ satisfying $f_{n}^{*}\left(\alpha_{i}, \alpha_{i+1}, \alpha_{i}, \ldots, \alpha_{i}\right)=2$ by 27. This implies $\left\{\alpha_{i}, \alpha_{i+1}\right\} \in$ $G_{2}$ if $\bmod (n, 2)=\bmod (i, 2)$. Next we will prove $\left\{\alpha_{i}, \alpha_{i+1}\right\} \notin G_{2}\left(f_{n}^{*}\right)$ if $\bmod (n, 2) \neq \bmod (i, 2)$. Since $\mathcal{X}^{n-2}=\left\{\boldsymbol{x}_{\backslash\{1,2\}} \mid \max \left\{\boldsymbol{x}_{\backslash\{1,2\}}\right\}<\alpha_{i+1}\right\} \bigcup\left\{\boldsymbol{x}_{\backslash\{1,2\}} \mid \max \left\{\boldsymbol{x}_{\backslash\{1,2\}}\right\}=\alpha_{i+1}\right\} \bigcup\left\{\boldsymbol{x}_{\backslash\{1,2\}} \mid \max \left\{\boldsymbol{x}_{\backslash\{1,2\}}\right\}>\right.$ $\left.\alpha_{i+1}\right\}$, it suffices to show that for any given $x_{1} \in \mathcal{X}$ and $\boldsymbol{x}_{\backslash\{1,2\}}$ in these three sets, the function will not differ when $\bmod (n, 2) \neq \bmod (i, 2)$. For $x_{1}<\max \left\{\boldsymbol{x}_{\backslash\{1,2\}}\right\}$ and $\boldsymbol{x}_{\backslash\{1,2\}} \in\left\{\boldsymbol{x}_{\backslash\{1,2\}} \mid \max \left\{\boldsymbol{x}_{\backslash\{1,2\}}\right\}<\alpha_{i+1}\right\}$, we observe that

$$
f_{n}^{*}\left(x_{1}, \alpha_{i}, \boldsymbol{x}_{\backslash\{1,2\}}\right) \stackrel{(a .1)}{=} f_{n-1}^{*}\left(\alpha_{i}, \boldsymbol{x} \backslash\{1,2\}\right)+1 \stackrel{(a .2)}{=} 2
$$

and

$$
f_{n}^{*}\left(x_{1}, \alpha_{i+1}, \boldsymbol{x}_{\backslash\{1,2\}}\right) \stackrel{(a .3)}{=} f_{n-1}^{*}\left(\alpha_{i+1}, \boldsymbol{x}_{\backslash\{1,2\}}\right)+1 \stackrel{(a .4)}{=} 2
$$

where (a.1) and (a.3) hold by (27), (a.2) hold by 25 if $\max \left\{\boldsymbol{x}_{\backslash\{1,2\}}\right\}<\alpha_{i}$, and by 26 and the fact that $\bmod (n, 2) \neq \bmod (i, 2)$ implies $\bmod (n-1,2)=\bmod (i, 2)$ if $\max \left\{\boldsymbol{x}_{\backslash\{1,2\}}\right\}=\alpha_{i}$, and (a.4) hold by (25). 
For $x_{1}<\max \left\{\boldsymbol{x}_{\backslash\{1,2\}}\right\}$ and $\boldsymbol{x}_{\backslash\{1,2\}} \in\left\{\boldsymbol{x}_{\backslash\{1,2\}} \mid \max \left\{\boldsymbol{x}_{\backslash\{1,2\}}\right\}=\alpha_{i+1}\right\}$, we observe that

$$
f_{n}^{*}\left(x_{1}, \alpha_{i}, \boldsymbol{x}_{\backslash\{1,2\}}\right) \stackrel{(b .1)}{=} f_{n-1}^{*}\left(\alpha_{i}, \boldsymbol{x} \backslash\{1,2\}\right)+1 \stackrel{(b .2)}{=} f_{n-2}^{*}\left(\boldsymbol{x}_{\backslash\{1,2\}}\right)+2
$$

and

$$
f_{n}^{*}\left(x_{1}, \alpha_{i+1}, \boldsymbol{x}_{\backslash\{1,2\}}\right) \stackrel{(b .3)}{=} f_{n-1}^{*}\left(\alpha_{i+1}, \boldsymbol{x}_{\backslash\{1,2\}}\right)+1 \stackrel{(b .4)}{=} f_{n-2}^{*}\left(\boldsymbol{x}_{\backslash\{1,2\}}\right)+2
$$

where (b.1) and (b.3) hold by by 277, (b.2) hold by [27, and (b.4) hold by 26 and the fact that $\bmod (n, 2) \neq$ $\bmod (i, 2)$ implies $\bmod (n-1,2) \neq \bmod (i+1,2)$. For $x_{1}<\max \left\{\boldsymbol{x}_{\backslash\{1,2\}}\right\}$ and $\boldsymbol{x}_{\backslash\{1,2\}} \in\left\{\boldsymbol{x}_{\backslash\{1,2\}} \mid \max \left\{\boldsymbol{x}_{\backslash\{1,2\}}\right\}>\right.$ $\left.\alpha_{i+1}\right\}$, we observe that

$$
f_{n}^{*}\left(x_{1}, \alpha_{i}, \boldsymbol{x}_{\backslash\{1,2\}}\right) \stackrel{(c .1)}{=} f_{n-1}^{*}\left(\alpha_{i}, \boldsymbol{x}_{\backslash\{1,2\}}\right)+1 \stackrel{(c .2)}{=} f_{n-2}^{*}\left(\boldsymbol{x}_{\backslash\{1,2\}}\right)+2
$$

and

$$
f_{n}^{*}\left(x_{1}, \alpha_{i+1}, \boldsymbol{x}_{\backslash\{1,2\}}\right) \stackrel{(c .3)}{=} f_{n-1}^{*}\left(\alpha_{i+1}, \boldsymbol{x}_{\backslash\{1,2\}}\right)+1 \stackrel{(c .4)}{=} f_{n-2}^{*}\left(\boldsymbol{x}_{\backslash\{1,2\}}\right)+2
$$

where (c.1) (c.2) (c.3) (c.4) all hold by 27. For $x_{1}=\max \left\{\boldsymbol{x}_{\backslash\{1,2\}}\right\}$ and $\boldsymbol{x}_{\backslash\{1,2\}} \in\left\{\boldsymbol{x}_{\backslash\{1,2\}} \mid \max \left\{\boldsymbol{x}_{\backslash\{1,2\}}\right\}<\right.$ $\left.\alpha_{i+1}\right\}$, we observe that

$$
f_{n}^{*}\left(x_{1}, \alpha_{i}, \boldsymbol{x}_{\backslash\{1,2\}}\right) \stackrel{(d .1)}{=} f_{n-1}^{*}\left(\alpha_{i}, \boldsymbol{x}_{\backslash\{1,2\}}\right)+1 \stackrel{(d .2)}{=} 2
$$

and

$$
f_{n}^{*}\left(x_{1}, \alpha_{i+1}, \boldsymbol{x}_{\backslash\{1,2\}}\right) \stackrel{(d .3)}{=} f_{n-1}^{*}\left(\alpha_{i+1}, \boldsymbol{x}_{\backslash\{1,2\}}\right)+1 \stackrel{(d .4)}{=} 2
$$

where (d.1) holds by 26) if $x_{1}=\alpha_{i}$ and by 27) if $x_{1}<\alpha_{i}$, (d.2) holds by 26] $\max \{\boldsymbol{x} \backslash\{1,2\}\}=\alpha_{i}$ and by $\left[25\right.$ if $\max \left\{\boldsymbol{x}_{\backslash\{1,2\}}\right\}<\alpha_{i}$, (d.3) holds by 27), and (d.4) holds by 25). For $x_{1}=\max \left\{\boldsymbol{x}_{\backslash\{1,2\}}\right\}$ and $\boldsymbol{x}_{\backslash\{1,2\}} \in\left\{\boldsymbol{x}_{\backslash\{1,2\}} \mid \max \left\{\boldsymbol{x}_{\backslash\{1,2\}}\right\}=\alpha_{i+1}\right\}$, we observe that

$$
f_{n}^{*}\left(x_{1}, \alpha_{i}, \boldsymbol{x}_{\backslash\{1,2\}} \stackrel{(e .1)}{=} 1\right.
$$

and

$$
f_{n}^{*}\left(x_{1}, \alpha_{i+1}, \boldsymbol{x}_{\backslash\{1,2\}}\right) \stackrel{(e .2)}{=} 1
$$

where (e.1) (e.2) both hold by by 26 and the fact that $\bmod (n, 2) \neq \bmod (i, 2)$ implies $\bmod (n, 2)=$ $\bmod (i+1,2)$. For $x_{1}=\max \left\{\boldsymbol{x}_{\backslash\{1,2\}}\right\}$ and $\boldsymbol{x}_{\backslash\{1,2\}} \in\left\{\boldsymbol{x}_{\backslash\{1,2\}} \mid \max \left\{\boldsymbol{x}_{\backslash\{1,2\}}\right\}>\alpha_{i+1}\right\}$, which means $x_{1}=$ $\max \{\boldsymbol{x}\}$, we observe that if $x_{1}=\alpha_{j}$ where $\bmod (j, 2)=\bmod (n, 2)$, then

$$
f_{n}^{*}\left(x_{1}, \alpha_{i}, \boldsymbol{x}_{\backslash\{1,2\}} \stackrel{(f .1)}{=} 1\right.
$$

and

$$
f_{n}^{*}\left(x_{1}, \alpha_{i+1}, \boldsymbol{x}_{\backslash\{1,2\}}\right) \stackrel{(f .2)}{=} 1
$$

where (f.1) (f.2) both hold by 26). If $x_{1}=\alpha_{j}$ where $\bmod (j, 2) \neq \bmod (n, 2)$, then

$$
f_{n}^{*}\left(x_{1}, \alpha_{i}, \boldsymbol{x}_{\backslash\{1,2\}}\right) \stackrel{(f .3)}{=} f_{n-1}^{*}\left(\alpha_{i}, \boldsymbol{x}_{\backslash\{1,2\}}\right)+1 \stackrel{(f .4)}{=} f_{n-2}^{*}\left(\boldsymbol{x}_{\backslash\{1,2\}}\right)+2
$$


and

$$
f_{n}^{*}\left(x_{1}, \alpha_{i+1}, \boldsymbol{x}_{\backslash\{1,2\}}\right) \stackrel{(f .5)}{=} f_{n-1}^{*}\left(\alpha_{i+1}, \boldsymbol{x}_{\backslash\{1,2\}}\right)+1 \stackrel{(f .6)}{=} f_{n-2}^{*}\left(\boldsymbol{x}_{\backslash\{1,2\}}\right)+2
$$

where (f.3) (f.4) both hold by [26, and (f.5) (f.6) both hold by [27). For $x_{1}>\max \left\{\boldsymbol{x}_{\backslash\{1,2\}}\right\}$ and $\boldsymbol{x}_{\backslash\{1,2\}} \in$ $\left\{\boldsymbol{x}_{\backslash\{1,2\}} \mid \max \left\{\boldsymbol{x}_{\backslash\{1,2\}}\right\}<\alpha_{i+1}\right\}$, we observe that if $x_{1} \leq \alpha_{i}$, then

$$
f_{n}^{*}\left(x_{1}, \alpha_{i}, \boldsymbol{x}_{\backslash\{1,2\}} \stackrel{(g .1)}{=} 2\right.
$$

and

$$
f_{n}^{*}\left(x_{1}, \alpha_{i+1}, \boldsymbol{x} \backslash\{1,2\}\right) \stackrel{(g .2)}{=} 2
$$

where (g.1) holds by (26) if $x_{1}=\alpha_{i}$, and by (27) if $x_{1}<\alpha_{i}$, and (g.2) holds by (27). If $x_{1}>\alpha_{i}$, then

$$
f_{n}^{*}\left(x_{1}, \alpha_{i}, \boldsymbol{x}_{\backslash\{1,2\}} \stackrel{(g .3)}{=} 1\right.
$$

and

$$
f_{n}^{*}\left(x_{1}, \alpha_{i+1}, \boldsymbol{x}_{\backslash\{1,2\}}\right) \stackrel{(g .4)}{=} 1
$$

where (g.3) holds by (25), and (g.4) holds by 26$)$ and the fact that $\bmod (n, 2) \neq \bmod (i, 2)$ implies $\bmod (n, 2)=\bmod (i+1,2)$ if $x_{1}=\alpha_{i+1}$, and by 25 if $x_{1}>\alpha_{i+1}$. For $x_{1}>\max \left\{\boldsymbol{x}_{\backslash\{1,2\}}\right\}$ and $\boldsymbol{x}_{\backslash\{1,2\}} \in$ $\left\{\boldsymbol{x}_{\backslash\{1,2\}} \mid \max \left\{\boldsymbol{x}_{\backslash\{1,2\}}\right\} \geq \alpha_{i+1}\right\}$, we observe that

$$
f_{n}^{*}\left(x_{1}, \alpha_{i}, \boldsymbol{x}_{\backslash\{1,2\}} \stackrel{\stackrel{(h .1)}{=}}{=} 1\right.
$$

and

$$
f_{n}^{*}\left(x_{1}, \alpha_{i+1}, \boldsymbol{x}_{\backslash\{1,2\}}\right) \stackrel{(h .2)}{=} 1
$$

where (h.1) (h.2) both hold by 25). Therefore in user 2's characteristic graph $G_{1}$, we have

$$
\left\{\alpha_{i}, \alpha_{j}\right\} \notin G_{2} \Longleftrightarrow \bmod (n, 2) \neq \bmod (i, 2) \& j=i+1 \text {. }
$$

By Lemma 3 and user 1 and 2's characteristic graphs, we know user $i$ 's characteristic graph will be complete for all $i \in[N] \backslash\{1,2\}$. Therefore if $N$ is odd and $L$ is odd, the set of all maximal independent sets for each of the users will be

$$
\begin{aligned}
& \Gamma(1)=\left\{\left\{\alpha_{1}, \alpha_{2}\right\},\left\{\alpha_{3}, \alpha_{4}\right\},\left\{\alpha_{5}, \alpha_{6}\right\}, \ldots,\left\{\alpha_{L-2}, \alpha_{L-1}\right\},\left\{\alpha_{L}\right\}\right\} \\
& \Gamma(2)=\left\{\left\{\alpha_{1}\right\},\left\{\alpha_{2}, \alpha_{3}\right\},\left\{\alpha_{4}, \alpha_{5}\right\}, \ldots,\{L-1, L\}\right\} \\
& \Gamma(i)=\left\{\left\{\alpha_{1}\right\},\left\{\alpha_{2}\right\}, \ldots,\left\{\alpha_{L}\right\}\right\} \forall i \in[N], i \notin\{1,2\} .
\end{aligned}
$$

If $N$ is odd and $L$ is even, the set of all maximal independent sets for each of the users will be

$$
\begin{aligned}
& \Gamma(1)=\left\{\left\{\alpha_{1}, \alpha_{2}\right\},\left\{\alpha_{3}, \alpha_{4}\right\},\left\{\alpha_{5}, \alpha_{6}\right\}, \ldots,\left\{\alpha_{L-1}, \alpha_{L}\right\}\right\} \\
& \Gamma(2)=\left\{\left\{\alpha_{1}\right\},\left\{\alpha_{2}, \alpha_{3}\right\},\left\{\alpha_{4}, \alpha_{5}\right\}, \ldots,\{L-2, L-1\},\{L\}\right\} \\
& \Gamma(i)=\left\{\left\{\alpha_{1}\right\},\left\{\alpha_{2}\right\}, \ldots,\left\{\alpha_{L}\right\}\right\} \forall i \in[N], i \notin\{1,2\} .
\end{aligned}
$$


If $N$ is even and $L$ is odd, the set of all maximal independent sets for each of the users will be

$$
\begin{aligned}
& \Gamma(1)=\left\{\left\{\alpha_{1}\right\},\left\{\alpha_{2}, \alpha_{3}\right\},\left\{\alpha_{4}, \alpha_{5}\right\}, \ldots,\left\{\alpha_{L-1}, \alpha_{L}\right\}\right\} \\
& \Gamma(2)=\left\{\left\{\alpha_{1}, \alpha_{2}\right\},\left\{\alpha_{3}, \alpha_{4}\right\}, \ldots,\{L-2, L-1\},\{L\}\right\} \\
& \Gamma(i)=\left\{\left\{\alpha_{1}\right\},\left\{\alpha_{2}\right\}, \ldots,\left\{\alpha_{L}\right\}\right\} \forall i \in[N], i \notin\{1,2\} .
\end{aligned}
$$

If $N$ is even and $L$ is even, the set of all maximal independent sets for each of the users will be

$$
\begin{aligned}
& \Gamma(1)=\left\{\left\{\alpha_{1}\right\},\left\{\alpha_{2}, \alpha_{3}\right\},\left\{\alpha_{4}, \alpha_{5}\right\}, \ldots,\left\{\alpha_{L-2}, \alpha_{L-1}\right\},\{L\}\right\} \\
& \Gamma(2)=\left\{\left\{\alpha_{1}, \alpha_{2}\right\},\left\{\alpha_{3}, \alpha_{4}\right\}, \ldots,\{L-1, L\}\right\} \\
& \Gamma(i)=\left\{\left\{\alpha_{1}\right\},\left\{\alpha_{2}\right\}, \ldots,\left\{\alpha_{L}\right\}\right\} \forall i \in[N], i \notin\{1,2\} .
\end{aligned}
$$

Note that in each $\Gamma(n), n \in[N]$, no vertex belongs to two maximal independent sets. Also note that $\left\{\alpha_{i}, \alpha_{i+1}\right\}$ appears exactly once in $\{\Gamma(n) \mid n \in[N]\}$ for all $\alpha_{i} \in \mathcal{X}$. To achieve the minimum sum-rate, the optimal coloring method would be assigning a color for each of the independent sets (see Fig. 5). For the case that both $L$ and $N$ are odd, we have

$$
\begin{aligned}
R_{A}\left(f_{n}^{*}\right) & =\sum_{i=1}^{n} \min _{c_{i} \in G_{i}\left(f_{n}^{*}\right)} H\left(c_{i}\left(X_{i}\right)\right) \\
& =(n-2) H(X)+\min _{c_{1} \in \mathcal{C}\left(G_{1}\left(f_{n}^{*}\right)\right)} H\left(c_{1}\left(X_{1}\right)+\min _{c_{2} \in \mathcal{C}\left(G_{2}\left(f_{n}^{*}\right)\right)} H\left(c_{2}\left(X_{2}\right)\right)\right. \\
& =-(n-2)\left(\sum_{i=1}^{L} p_{i} \log _{2} p_{i}\right)-\left(\sum_{i=1}^{\frac{L-1}{2}} p_{2 i-1,2 i} \log _{2} p_{2 i-1,2 i}\right)-p_{L} \log _{2} p_{L}-p_{1} \log _{2} p_{1}-\left(\sum_{i=1}^{\frac{L-1}{2}} p_{2 i, 2 i+1} \log _{2} p_{2 i, 2 i+1}\right) \\
& =-(n-2)\left(\sum_{i=1}^{L} p_{i} \log _{2} p_{i}\right)-\left(\sum_{i=1}^{L-1} p_{i, i+1} \log _{2} p_{i, i+1}\right)-p_{1} \log _{2} p_{1}-p_{L} \log _{2} p_{L} .
\end{aligned}
$$

For the other cases, we will get the exact same expression of the sum-rate although there exists a minor variation on the argument.

Now we will show that for any function $f_{n} \in \mathcal{F}_{A, n}, n \in[N]$ the sum-rate under $f_{n}$ will be no lower than 28 and $f_{n}^{*} \in \mathcal{F}_{A, n}^{*}$. By Lemma 2 we know that no three vertices can be assigned the same color and hence only the neighbor pair can share the color. By applying Lemma 3 N-1 times, we know that if a neighbor pair $\left\{\alpha_{i}, \alpha_{i+1}\right\}$ are given the same color in user 1's characteristic graph then they have to have distinct colors in all other users' graph. Therefore for all candidate arg max functions, we can have at most $L-1$ different consecutive pairs $\left\{\alpha_{1}, \alpha_{2}\right\}, \cdots,\left\{\alpha_{L-1}, \alpha_{L}\right\}$ that share the color, all other vertices have to have their own colors, and by assigning distinct colors to each of the $L-1$ node paris and all other single nodes and encoding the colors by SW coding, 28) is achieved. 
2) Proof of Theorem 7. Proof: By Theorem 2 we have

$$
\begin{aligned}
\Delta_{A} & =N H(X)-(N-2) H(X)+\sum_{i=1}^{L-1}\left(p_{i, i+1} \log _{2} p_{i, i+1}\right)+p_{1} \log _{2} p_{1}+p_{L} \log _{2} p_{L} \\
& =-2 \sum_{i=1}^{L}\left(p_{i} \log _{2} p_{i}\right)+\sum_{i=1}^{L-1}\left(p_{i} \log _{2} p_{i, i+1}\right)+\sum_{i=1}^{L-1}\left(p_{i+1} \log _{2} p_{i, i+1}\right)+p_{1} \log _{2} p_{1}+p_{L} \log _{2} p_{L} \\
& =-\sum_{i=1}^{L-1}\left(p_{i} \log _{2} p_{i}\right)-\sum_{i=2}^{L}\left(p_{i} \log _{2} p_{i}\right)+\sum_{i=1}^{L-1}\left(p_{i} \log _{2} p_{i, i+1}\right)+\sum_{i=1}^{L-1}\left(p_{i+1} \log _{2} p_{i, i+1}\right) \\
& =-\sum_{i=1}^{L-1}\left(p_{i} \log _{2} \frac{p_{i}}{p_{i, i+1}}\right)-\sum_{i=1}^{L-1}\left(p_{i+1} \log _{2} \frac{p_{i+1}}{p_{i, i+1}}\right) \\
& =\sum_{i=1}^{L-1}\left(p_{i}+p_{i+1}\right) h_{2}\left(\frac{p_{i}}{p_{i}+p_{i+1}}\right) \\
& \leq \sum_{i=1}^{L-1}\left(p_{i}+p_{i+1}\right)<2 \sum_{i=1}^{L} p_{i}=2 .
\end{aligned}
$$

Hence,

$$
\lim _{N \rightarrow \infty} \frac{\Delta_{A}}{N}=0
$$

3) Proof of Corollary 3. Proof: By Theorem 4, we get no savings if $\min \mathcal{X}>0$. By Theorem 5 if $\min \mathcal{X}=0$, we have

$$
\begin{aligned}
\frac{\Delta_{M}}{N} & =N H(X)-\sum_{n=1}^{N} H_{G}\left(X_{n}\right) \\
& =N\left(p_{1}+p_{2}\right) h_{2}\left(\frac{p_{1}}{p_{1}+p_{2}}\right)
\end{aligned}
$$

and

$$
\lim _{N \rightarrow \infty} \frac{\Delta_{M}}{N}=\left(p_{1}+p_{2}\right) h_{2}\left(\frac{p_{1}}{p_{1}+p_{2}}\right) .
$$

4) Proof of Theorem 9. Proof: The optimal Bayes estimator will select one of the users that reports being in the highest interval.

$$
n_{j} \triangleq \sum_{i=1}^{N} \mathbb{1}_{\ell_{j-1} \leq X_{i} \leq \ell_{j}} .
$$

We then have

$$
\begin{aligned}
\mathbb{E}\left[X_{\hat{Z}_{A}}\right] & =\sum_{j=1}^{K}\left[E_{j} \sum_{\substack{\sum_{k}^{j} n_{k}=N \\
n_{j}>0}}\left(\begin{array}{c}
N \\
n_{1}, \ldots, n_{j}
\end{array}\right) p_{1}^{n_{1}} \cdots p_{j}^{n_{j}}\right] \\
& =\sum_{j=1}^{K}\left[E_{j}\left(\left(\sum_{k=1}^{j} p_{k}\right)^{N}-\left(\sum_{k=1}^{j-1} p_{k}\right)^{N}\right)\right] \\
& =\sum_{j=1}^{K}\left[E_{j}\left(F_{j}^{N}-F_{j-1}^{N}\right)\right] .
\end{aligned}
$$


The last step follows from observing

$$
\sum_{\sum_{k}^{j} n_{k}=N}\left(\begin{array}{c}
N \\
n_{1}, \ldots, n_{j}
\end{array}\right) p_{1}^{n_{1}} \cdots p_{j}^{n_{j}}=\sum_{\substack{\sum_{k}^{j} n_{k}=N \\
n_{j}>0}}\left(\begin{array}{c}
N \\
n_{1}, \ldots, n_{j}
\end{array}\right) p_{1}^{n_{1}} \cdots p_{j}^{n_{j}}+\sum_{\substack{\sum_{k}^{j} n_{k}=N \\
n_{j}=0}}\left(\begin{array}{c}
N \\
n_{1}, \ldots, n_{j}
\end{array}\right) p_{1}^{n_{1}} \cdots p_{j}^{n_{j}}
$$

rearranging and applying the multinomial theorem yeilds

$$
\sum_{\substack{\sum_{k}^{j} n_{k}=N \\
n_{j}>0}}\left(\begin{array}{c}
N \\
n_{1}, \ldots, n_{j}
\end{array}\right) p_{1}^{n_{1}} \cdots p_{j}^{n_{j}}=\left(\sum_{k=1}^{j} p_{k}\right)^{N}-\left(\sum_{k=1}^{j-1} p_{k}\right)^{N}
$$

5) Proof of Lemma 77 $\quad$ Proof: We re-write (119) as

$$
\begin{aligned}
\mathbb{E}\left[X_{\hat{Z}_{A}}\right] & =\sum_{j=1}^{K}\left[E_{j}\left(F_{j}^{N}-F_{j-1}^{N}\right)\right] \\
& =F_{K}^{N} E_{K}-\sum_{j=1}^{K-1} F_{j}^{N}\left(E_{j+1}-E_{j}\right)-F_{0}^{N} E_{1} \\
& =E_{K}-\sum_{j=1}^{K-1} F_{j}^{N}\left(E_{j+1}-E_{j}\right)
\end{aligned}
$$

and take derivatives

$$
\frac{\partial \mathbb{E}\left[X_{\hat{Z}_{A}}\right]}{\partial \ell_{k}}=\frac{\partial}{\partial \ell_{k}} E_{K}-\sum_{j=1}^{K-1} \frac{\partial}{\partial \ell_{k}} F_{j}^{N}\left(E_{j+1}-E_{j}\right) .
$$

If $k \neq K-1$, the above becomes

$$
\begin{aligned}
\frac{\partial \mathbb{E}\left[X_{\left.\hat{Z}_{A}\right]}\right]}{\partial \ell_{k}} & =-\sum_{j=1}^{K-1} \frac{\partial}{\partial \ell_{k}} F_{j}^{N}\left(E_{j+1}-E_{j}\right) \\
& =-\frac{\partial}{\partial \ell_{k}} F_{k-1}^{N}\left(E_{k}-E_{k-1}\right)-\frac{\partial}{\partial \ell_{k}} F_{k}^{N}\left(E_{k+1}-E_{k}\right)-\frac{\partial}{\partial \ell_{k}} F_{k+1}^{N}\left(E_{k+2}-E_{k+1}\right) \\
& =-F_{k-1}^{N} \frac{\partial E_{k}}{\partial \ell_{k}}-N F_{k}^{N-1} f_{k}\left(E_{k+1}-E_{k}\right)-F_{k}^{N}\left(\frac{\partial E_{k+1}}{\partial \ell_{k}}-\frac{\partial E_{k}}{\partial \ell_{k}}\right)+F_{k+1}^{N} \frac{\partial E_{k+1}}{\partial \ell_{k}} \\
& =f_{k}\left[-F_{k-1}^{N} \frac{\ell_{k}-E_{k}}{p_{k}}-N F_{k}^{N-1}\left(E_{k+1}-E_{k}\right)-F_{k}^{N}\left(\frac{E_{k+1}-\ell_{k}}{p_{k+1}}-\frac{\ell_{k}-E_{k}}{p_{k}}\right)+F_{k+1}^{N} \frac{E_{k+1}-\ell_{k}}{p_{k+1}}\right]
\end{aligned}
$$

If $k=K-1$, the above becomes

$$
\begin{aligned}
\frac{\partial \mathbb{E}\left[X_{\hat{Z}_{A}}\right]}{\partial \ell_{K-1}} & =\frac{\partial}{\partial \ell_{K-1}} E_{K}-\frac{\partial}{\partial \ell_{K-1}} F_{K-2}^{N}\left(E_{K-1}-E_{K-2}\right)-\frac{\partial}{\partial \ell_{K-1}} F_{K-1}^{N}\left(E_{K}-E_{K-1}\right) \\
& =-F_{K-2}^{N} \frac{\partial E_{K-1}}{\partial \ell_{K-1}}-N F_{K-1}^{N-1} f_{K-1}\left(E_{K}-E_{K-1}\right)-F_{K-1}^{N}\left(\frac{\partial E_{K}}{\partial \ell_{K-1}}-\frac{\partial E_{K-1}}{\partial \ell_{K-1}}\right)+F_{K}^{N} \frac{\partial E_{K}}{\partial \ell_{K-1}}
\end{aligned}
$$

The above follows from recognizing that $F_{K}=1$ and we see that the expression for $k \neq K-1$ holds for $k=K-1$. 
6) Proof of Corollary 6r Proof:

$$
\begin{aligned}
\frac{\partial \mathbb{E}\left[X_{\hat{Z}_{A}}\right]}{\partial \ell_{k}} & =f_{k}\left[\frac{\left(F_{k+1}^{2}-F_{k}^{2}\right)\left(E_{k+1}-\ell_{k}\right)}{p_{k+1}}+\frac{\left(F_{k}^{2}-F_{k-1}^{2}\right)\left(\ell_{k}-E_{k}\right)}{p_{k}}-2 F_{k}\left(E_{k+1}-E_{k}\right)\right] \\
& =f_{k}\left[\frac{\left(F_{k+1}^{2}-F_{k}^{2}\right)\left(E_{k+1}-\ell_{k}\right)}{F_{k+1}-F_{k}}+\frac{\left(F_{k}^{2}-F_{k-1}^{2}\right)\left(\ell_{k}-E_{k}\right)}{F_{k}-F_{k-1}}-2 F_{k}\left(E_{k+1}-E_{k}\right)\right] \\
& =f_{k}\left[\left(F_{k+1}+F_{k}\right)\left(E_{k+1}-\ell_{k}\right)+\left(F_{k}+F_{k-1}\right)\left(\ell_{k}-E_{k}\right)-2 F_{k}\left(E_{k+1}-E_{k}\right)\right] \\
& =f_{k}\left[\left(F_{k+1}-F_{k}\right) E_{k+1}+\left(F_{k}-F_{k-1}\right) E_{k}-\left(F_{k+1}-F_{k-1}\right) \ell_{k}\right] \\
& =f_{k}\left[\int_{\ell_{k}}^{\ell_{k+1}} x f(x) \mathrm{d} x+\int_{\ell_{k-1}}^{\ell_{k}} x f(x) \mathrm{d} x-\ell_{k} \int_{\ell_{k-1}}^{\ell_{k+1}} f(x) \mathrm{d} x\right]
\end{aligned}
$$

7) Proof of Theorem 13 Proof: For an arg max quantizer, an average distortion is

$$
\begin{aligned}
\mathbb{E}\left[D\left(\left(X_{1}, X_{2}\right), \hat{z}\right)\right] & =\mathbb{E}\left[\mathbb{E}\left[D\left(\left(X_{1}, X_{2}\right), \hat{z}\right) \mid U_{1}, U_{2}\right]\right] \\
& =\sum_{k_{1}, k_{2}} \mathbb{E}\left[Z_{M}-X_{\hat{z}} \mid U_{1}=k_{1}, U_{2}=k_{2}\right] \mathbb{P}\left(U_{1}=k_{1}, U_{2}=k_{2}\right) \\
& =\sum_{\left(k_{1}, k_{2}\right) \in \mathbf{Z}_{1}} \mathbb{E}\left[X_{1}-X_{\hat{z}} \mid X_{1} \in \mathcal{L}_{1, k_{1}}, X_{2} \in \mathcal{L}_{2, k_{2}}\right] \mathbb{P}\left(X_{1} \in \mathcal{L}_{1, k_{1}}, X_{2} \in \mathcal{L}_{2, k_{2}}\right) \\
& +\sum_{\left(k_{1}, k_{2}\right) \in \mathbf{Z}_{2}} \mathbb{E}\left[X_{2}-X_{\hat{z}} \mid X_{1} \in \mathcal{L}_{1, k_{1}}, X_{2} \in \mathcal{L}_{2, k_{2}}\right] \mathbb{P}\left(X_{1} \in \mathcal{L}_{1, k_{1}}, X_{2} \in \mathcal{L}_{2, k_{2}}\right) \\
& +\sum_{\left(k_{1}, k_{2}\right) \in \mathbf{Z}_{0}} \mathbb{E}\left[X_{Z_{A}}-X_{\hat{z}} \mid X_{1} \in \mathcal{L}_{1, k_{1}}, X_{2} \in \mathcal{L}_{2, k_{2}}\right] \mathbb{P}\left(X_{1} \in \mathcal{L}_{1, k_{1}}, X_{2} \in \mathcal{L}_{2, k_{2}}\right)
\end{aligned}
$$

where $\mathbf{Z}_{1}=\left\{\left(k_{1}, k_{2}\right): \ell_{2, k_{2}} \leq \ell_{1, k_{1}-1}\right\}, \mathbf{Z}_{2}=\left\{\left(k_{1}, k_{2}\right): \ell_{1, k_{1}} \leq \ell_{2, k_{2}-1}\right\}$, and $\mathbf{Z}_{0}=\left\{\left(k_{1}, k_{2}\right): \max \left(\ell_{1, k_{1}-1}, \ell_{2, k_{2}-1}\right) \leq\right.$ $\left.\min \left(\ell_{1, k_{1}}, \ell_{2, k_{2}}\right) \leq \max \left(\ell_{1, k_{1}}, \ell_{2, k_{2}}\right)\right\}$. Observe that

$$
\begin{aligned}
\hat{z}\left(U_{1}, U_{2}\right) & =\underset{z}{\arg \min } \mathbb{E}\left[d\left(\left(X_{1}, X_{2}\right), z\right) \mid U_{1}=k_{1}, U_{2}=k_{2}\right] \\
& =\underset{z}{\arg \min } \mathbb{E}\left[Z_{M}-X_{z} \mid X_{1} \in \mathcal{L}_{1, k_{1}}, X_{2} \in \mathcal{L}_{2, k_{2}}\right] \\
& =\underset{z}{\arg \max } \mathbb{E}\left[X_{z} \mid X_{1} \in \mathcal{L}_{1, k_{1}}, X_{2} \in \mathcal{L}_{2, k_{2}}\right] .
\end{aligned}
$$

When $\left(U_{1}, U_{2}\right) \in \mathbf{Z}_{1}, Z_{A}=1$ and a distortion of 0 can be attained with $\hat{z}=1$. When $\left(U_{1}, U_{2}\right) \in \mathbf{Z}_{2}, Z_{A}=2$ and a distortion of 0 can be attained with $\hat{z}=2$. For region $\mathbf{Z}_{0}, Z_{M}$ may be equal to either $X_{1}$ or $X_{2}$ and we see from 249] that the Bayes estimator is

$$
\hat{z}\left(U_{1}, U_{2}\right)= \begin{cases}1 & \text { if } \mathbb{E}\left[X_{1} \mid X_{1} \in \mathcal{L}_{1, U_{1}}\right] \geq \mathbb{E}\left[X_{2} \mid X_{2} \in \mathcal{L}_{2, U_{2}}\right] \\ 2 & \text { otherwise. }\end{cases}
$$

To derive the expression for the average distortion in the region $\mathbf{Z}_{0}$, we partition into the two sets $\mathbf{Z}_{01}$ and $\mathbf{Z}_{02}$ and break the last summation in 248) into two parts and substitute the appropriate conditional density functions and the expression for the Bayes estimator. 
8) Proof of Theorem 14. Proof: For a max quantizer, the average distortion can be expressed as follows

$$
\begin{aligned}
\mathbb{E}\left[d\left(\left(X_{1}, X_{2}\right), \hat{z}\right)\right] & =\mathbb{E}\left[\mathbb{E}\left[d\left(\left(\left(X_{1}, X_{2}\right), \hat{z}\left(U_{1}, U_{2}\right)\right) \mid U_{1}, U_{2}\right]\right]\right. \\
& =\sum_{k_{1}, k_{2}} \mathbb{E}\left[d\left(\left(\left(X_{1}, X_{2}\right), \hat{z}\right) \mid U_{1}=k_{1}, U_{2}=k_{2}\right] \mathbb{P}\left(U_{1}=k_{1}, U_{2}=k_{2}\right)\right. \\
& =\sum_{\left(k_{1}, k_{2}\right) \in \mathbf{Z}_{1}} \mathbb{E}\left[d\left(X_{1}, \hat{z}\right) \mid X_{1} \in \mathcal{L}_{1, k_{1}}, X_{2} \in \mathcal{L}_{2, k_{2}}\right] \mathbb{P}\left(X_{1} \in \mathcal{L}_{1, k_{1}}, X_{2} \in \mathcal{L}_{2, k_{2}}\right) \\
& +\sum_{\left(k_{1}, k_{2}\right) \in \mathbf{Z}_{2}} \mathbb{E}\left[d\left(X_{2}, \hat{z}\right) \mid X_{1} \in \mathcal{L}_{1, k_{1}}, X_{2} \in \mathcal{L}_{2, k_{2}}\right] \mathbb{P}\left(X_{1} \in \mathcal{L}_{1, k_{1}}, X_{2} \in \mathcal{L}_{2, k_{2}}\right) \\
& +\sum_{\left(k_{1}, k_{2}\right) \in \mathbf{Z}_{0}} \mathbb{E}\left[d\left(Z_{M}, \hat{z}\right) \mid X_{1} \in \mathcal{L}_{1, k_{1}}, X_{2} \in \mathcal{L}_{2, k_{2}}\right] \mathbb{P}\left(X_{1} \in \mathcal{L}_{1, k_{1}}, X_{2} \in \mathcal{L}_{2, k_{2}}\right)
\end{aligned}
$$

In order to find a quantizer minimizing the average distortion, we need to evaluate a minimum distortion term as follows,

$$
\begin{aligned}
\hat{z}\left(U_{1}, U_{2}\right) & =\underset{z}{\arg \min } \mathbb{E}\left[d\left(Z_{M}, z\right) \mid U_{1}=k_{1}, U_{2}=k_{2}\right] \\
& =\underset{z}{\arg \min } \mathbb{E}\left[Z_{M}-\hat{z} \mathbb{1}_{Z_{M} \geq z} \mid X_{1} \in \mathcal{L}_{1, k_{1}}, X_{2} \in \mathcal{L}_{2, k_{2}}\right] \\
& =\underset{z}{\arg \max } z \mathbb{P}\left(Z_{M} \geq z \mid X_{1} \in \mathcal{L}_{1, k_{1}}, X_{2} \in \mathcal{L}_{2, k_{2}}\right)
\end{aligned}
$$

When $\left(U_{1}, U_{2}\right) \in \mathbf{Z}_{1}$, then $Z_{M}=X_{1}$ and we have

$$
\hat{z}\left(U_{1}, U_{2}\right)=\underset{z}{\arg \max } z \mathbb{P}\left(X_{1} \geq z \mid X_{1} \in \mathcal{L}_{1, k_{1}}\right)
$$

In order to find an optimum estimation $\hat{z}_{1}^{*}$ minimizing the average distortion in region $\mathbf{Z}_{1}$, the necessary and sufficient condition is to find $z$ maximizing

$$
z \mathbb{P}\left(X_{1} \geq z \mid X_{1} \in \mathcal{L}_{1, k_{1}}\right)= \begin{cases}z & z \leq \ell_{1, k_{1}-1} \\ z \frac{F_{X}\left(\ell_{1, k_{1}}\right)-F_{X}(z)}{F_{X}\left(\ell_{1, k_{1}}\right)-F_{X}\left(\ell_{1, k_{1}-1}\right)} & z \in \mathcal{L}_{1, k_{1}} \\ 0 & z \geq \ell_{1, k_{1}}\end{cases}
$$

Since the maximum of each region can be included on the boundary from $\mathcal{L}_{1, k_{1}}$, it is suffices to evaluate as follows,

$$
\hat{z}_{1}^{*}=\underset{z \in \mathcal{L}_{1, k_{1}}}{\arg \max } z \frac{F_{X}\left(\ell_{1, k_{1}}\right)-F_{X}(z)}{F_{X}\left(\ell_{1, k_{1}}\right)-F_{X}\left(\ell_{1, k_{1}-1}\right)}
$$

If it is possible to take the first and second derivative of 254 with respect to $z$, the estimation $\hat{z}_{1}^{*}$ can be determined by

$$
\hat{z}_{1}^{*}= \begin{cases}\operatorname{sol}\left\{z: F_{X}\left(\ell_{1, k_{1}}\right)=F_{X}(z)+z f_{X}(z), 2 f_{X}(z)+z f_{X}^{\prime}(z) \geq 0\right\} & z \in \mathcal{L}_{1, k_{1}} \\ \ell_{1, k_{1}-1} & \text { otherwise }\end{cases}
$$

In order to find an average distortion in the region $\mathbf{Z}_{1}$, we first give $\mathbb{E}\left[X_{1} \mid X_{1} \in \mathcal{L}_{1, k_{1}}\right]$ as follows

$$
\mathbb{E}\left[X_{1} \mid X_{1} \in \mathcal{L}_{1, k_{1}}\right]=\int_{\ell_{1, k_{1}-1}}^{\ell_{1, k_{1}}} x \frac{f_{X}(x)}{F_{X}\left(\ell_{1, k_{1}}\right)-F_{X}\left(\ell_{1, k_{1}-1}\right)} \mathrm{d} x .
$$


The average conditional minimum distortion when $\left(U_{1}, U_{2}\right) \in \mathbf{Z}_{1}$ is

$$
\begin{aligned}
\mathbb{E}\left[X_{1} \mid X_{1} \in \mathcal{L}_{1, k_{1}}\right]-\hat{z}_{1}^{*} \mathbb{P}\left(X_{1} \geq\right. & \left.\hat{z}_{1}^{*} \mid X_{1} \in \mathcal{L}_{1, k_{1}}\right)= \\
& \int_{\ell_{1, k_{1}-1}}^{\ell_{1, k_{1}}} x \frac{f_{X}(x)}{F_{X}\left(\ell_{1, k_{1}}\right)-F_{X}\left(\ell_{1, k_{1}-1}\right)} \mathrm{d} x-\hat{z}_{1}^{*} \frac{F_{X}\left(\ell_{1, k_{1}}\right)-F_{X}\left(\hat{z}_{1}^{*}\right)}{F_{X}\left(\ell_{1, k_{1}}\right)-F_{X}\left(\ell_{1, k_{1}-1}\right)}
\end{aligned}
$$

Therefore, the average distortion in $\mathbf{Z}_{1}$ is,

$$
\begin{aligned}
\sum_{\left(k_{1}, k_{2}\right) \in \mathbf{Z}_{1}} \mathbb{E}\left[d\left(X_{1}, \hat{z}\right) \mid X_{1} \in \mathcal{L}_{1, k_{1}}, X_{2} \in \mathcal{L}_{2, k_{2}}\right] \mathbb{P}\left(X_{1} \in \mathcal{L}_{1, k_{1}}, X_{2} \in \mathcal{L}_{2, k_{2}}\right)= \\
\sum_{\left(k_{1}, k_{2}\right) \in \mathbf{Z}_{1}}\left[\int_{\ell_{1, k_{1}-1}}^{\ell_{1, k_{1}}} x f_{X}(x) \mathrm{d} x-\hat{z}_{1}^{*}\left(F_{X}\left(\ell_{1, k_{1}}\right)-F_{X}\left(\hat{z}_{1}^{*}\right)\right)\right]\left(F_{X}\left(\ell_{2, k_{2}}\right)-F_{X}\left(\ell_{2, k_{2}-1}\right)\right)
\end{aligned}
$$

When $\left(U_{1}, U_{2}\right) \in \mathbf{Z}_{2}$, then $Z_{M}=X_{2}$ and by symmetry we have that the estimation $\hat{z}_{2}^{*}$ minimizing the average distortion in region $\mathbf{Z}_{2}$

$$
\hat{z}_{2}^{*}=\underset{z}{\arg \max } z \mathbb{P}\left(X_{2} \geq z \mid X_{2} \in \mathcal{L}_{2, k_{2}}\right)
$$

If is is possible to take the first and second derivatives, then $\hat{z}_{2}^{*}$ can be determined by

$$
\hat{z}_{2}^{*}= \begin{cases}\operatorname{sol}\left\{z \mid F_{X}\left(\ell_{2, k_{2}}\right)=F_{X}(z)+z f_{X}(z), 2 f_{X}(z)+z f_{X}^{\prime}(z) \geq 0\right\} & z \in \mathcal{L}_{2, k_{2}} \\ \ell_{2, k_{2}-1} & \text { otherwise }\end{cases}
$$

The average conditional minimum distortion when $\left(U_{1}, U_{2}\right) \in \mathbf{Z}_{2}$ is

$$
\begin{aligned}
\mathbb{E}\left[X_{2} \mid X_{2} \in \mathcal{L}_{2, k_{2}}\right]-\hat{z}_{2}^{*} \mathbb{P}\left(X_{2} \geq\right. & \left.\hat{z}_{2}^{*} \mid X_{2} \in \mathcal{L}_{2, k_{2}}\right)= \\
& \int_{\ell_{2, k_{2}-1}}^{\ell_{2, k_{2}}} x \frac{f_{X}(x)}{F_{X}\left(\ell_{2, k_{2}}\right)-F_{X}\left(\ell_{2, k_{2}-1}\right)} \mathrm{d} x-\hat{z}_{2}^{*} \frac{F_{X}\left(\ell_{2, k_{2}}\right)-F_{X}\left(\hat{z}_{2}^{*}\right)}{F_{X}\left(\ell_{2, k_{2}}\right)-F_{X}\left(\ell_{2, k_{2}-1}\right)}
\end{aligned}
$$

Therefore, the average distortion in region $\mathbf{Z}_{2}$ is

$$
\begin{aligned}
& \sum_{\left(k_{1}, k_{2}\right) \in \mathbf{Z}_{2}} \mathbb{E}\left[d\left(X_{2}, \hat{z}\right) \mid X_{1} \in \mathcal{L}_{1, k_{1}}, X_{2} \in \mathcal{L}_{2, k_{2}}\right] \mathbb{P}\left(X_{1} \in \mathcal{L}_{1, k_{1}}, X_{2} \in \mathcal{L}_{2, k_{2}}\right)= \\
& \sum_{\left(k_{1}, k_{2}\right) \in \mathbf{Z}_{2}}\left[\int_{\ell_{2, k_{2}-1}}^{\ell_{2, k_{2}}} x f_{X}(x) \mathrm{d} x-\hat{z}_{2}^{*}\left(F_{X}\left(\ell_{2, k_{2}}\right)-F_{X}\left(\hat{z}_{2}^{*}\right)\right)\right]\left(F_{X}\left(\ell_{1, k_{1}}\right)-F_{X}\left(\ell_{1, k_{1}-1}\right)\right)
\end{aligned}
$$

When $\left(U_{1}, U_{2}\right) \in \mathbf{Z}_{0}$, the maximum value $Z_{M}$ may be equal to either $X_{1}$ or $\left.X_{2}\right)$ and

$$
\hat{z}_{0}^{*}=\underset{z}{\arg \max } z \mathbb{P}\left(Z_{M} \geq z \mid X_{1} \in \mathcal{L}_{1, k_{1}}, X_{2} \in \mathcal{L}_{2, k_{2}}\right) .
$$

For the case of $\left(U_{1}, U_{2}\right) \in \mathbf{Z}_{01} \subseteq \mathbf{Z}_{0}$ (i.e., $\left.\max \left(\ell_{2, k_{2}-1}, \ell_{1, k_{1}-1}\right)<\ell_{2, k_{2}} \leq \ell_{1, k_{1}}\right)$, the CDF of $Z_{M}$ given 
$X_{1} \in \mathcal{L}_{1, k_{1}}$ and $X_{2} \in \mathcal{L}_{2, k_{2}}$ is

$$
\begin{aligned}
F_{Z_{M} \mid X_{1} \in \mathcal{L}_{1, k_{1}}, X_{2} \in \mathcal{L}_{2, k_{2}}}(z) & =F_{X \mid X \in \mathcal{L}_{1, k_{1}}(z) F_{X \mid X \in \mathcal{L}_{2, k_{2}}}(z)} \\
& = \begin{cases}0 & z \leq \max \left(\ell_{1, k_{1}-1}, \ell_{2, k_{2}-1}\right) \\
F_{X \mid X \in \mathcal{L}_{1, k_{1}}}(z) F_{X \mid X \in \mathcal{L}_{2, k_{2}}}(z) & \max \left(\ell_{1, k_{1}-1}, \ell_{2, k_{2}-1}\right) \leq z \leq \ell_{2, k_{2}} \\
F_{X \mid X \in \mathcal{L}_{1, k_{1}}}(z) & \ell_{2, k_{2}} \leq z \leq \ell_{1, k_{1}} \\
1 & z \geq \ell_{1, k_{1}}\end{cases}
\end{aligned}
$$

then, $z \mathbb{P}\left(Z_{M} \geq z \mid X_{1} \in \mathcal{L}_{1, k_{1}}, X_{2} \in \mathcal{L}_{2, k_{2}}\right)$ is

$$
z \mathbb{P}\left(Z_{M} \geq z \mid X_{1} \in \mathcal{L}_{1, k_{1}}, X_{2} \in \mathcal{L}_{2, k_{2}}\right)= \begin{cases}z & z \leq \max \left(\ell_{1, k_{1}-1}, \ell_{2, k_{2}-1}\right) \\ z\left[1-F_{X \mid X \in \mathcal{L}_{1, k_{1}}}(z) F_{X \mid X \in \mathcal{L}_{2, k_{2}}}(z)\right] & \max \left(\ell_{1, k_{1}-1}, \ell_{2, k_{2}-1}\right) \leq z \leq \ell_{2, k_{2}} \\ z\left[1-F_{X \mid X \in \mathcal{L}_{1, k_{1}}}(z)\right] & \ell_{2, k_{2}} \leq z \leq \ell_{1, k_{1}} \\ 0 & z \geq \ell_{1, k_{1}}\end{cases}
$$

Since the maximum of each region can be also included on the boundary from $\max \left(\ell_{1, k_{1}-1}, \ell_{2, k_{2}-1}\right) \leq \hat{z} \leq \ell_{1, k_{1}}$, it is suffices to evaluate as follows,

$$
\hat{z}_{01}^{*}=\underset{z}{\arg \max } \begin{cases}w_{11}(z) & \max \left(\ell_{1, k_{1}-1}, \ell_{2, k_{2}-1}\right) \leq z \leq \ell_{2, k_{2}} \\ w_{12}(z) & \ell_{2, k_{2}} \leq z \leq \ell_{1, k_{1}} .\end{cases}
$$

where

$$
\begin{aligned}
& w_{11}(z)=z\left(1-F_{X \mid X \in \mathcal{L}_{1, k_{1}}}(z) F_{X \mid X \in \mathcal{L}_{2, k_{2}}}(z)\right) \\
& w_{12}(z)=z\left(1-F_{X \mid X \in \mathcal{L}_{1, k_{1}}}(z)\right)
\end{aligned}
$$

If we can take first and second derivatives, then for $\max \left(\ell_{1, k_{1}-1}, \ell_{2, k_{2}-1}\right) \leq z \leq \ell_{2, k_{2}}$, a condition such that $w_{11}^{\prime}(\hat{z})=0$ is

$$
\begin{aligned}
\left(z f_{X}(z)+F_{X}(z)-F_{X}\left(\ell_{1, k_{1}}\right)\right) F_{X}\left(\ell_{2, k_{2}-1}\right)+\left(z f_{X}(z)\right. & \left.+F_{X}(z)-F_{X}\left(\ell_{2, k_{2}}\right)\right) F_{X}\left(\ell_{1, k_{1}-1}\right)= \\
& +2 z F(x) f(x)+F_{X}^{2}(z)-F_{X}\left(\ell_{1, k_{1}}\right) F_{X}\left(\ell_{2, k_{2}}\right)
\end{aligned}
$$

and $w_{11}^{\prime \prime}(z) \leq 0$ is

$$
\left(2 f_{X}(z)+z f_{X}^{\prime}(z)\right)\left(F_{X}\left(\ell_{2, k_{2}-1}\right)-F_{X}\left(\ell_{1, k_{1}-1}\right)-2 F_{X}(z)\right) \leq 2 z f_{X}^{2}(z)
$$

A maximizer $\hat{z}_{11}^{*}$ for $w_{11}(z)$ is given by

$$
\hat{z}_{11}^{*}= \begin{cases}\operatorname{sol}\left\{z: w_{11}^{\prime}(z)=0, w_{11}^{\prime \prime}(z) \leq 0\right\} & \max \left(\ell_{1, k_{1}-1}, \ell_{2, k_{2}-1}\right) \leq z \leq \ell_{2, k_{2}} \\ \max \left(\ell_{1, k_{1}-1}, \ell_{2, k_{2}-1}\right) & \text { otherwise }\end{cases}
$$


For $\ell_{2, k_{2}} \leq z \leq \ell_{1, k_{1}}$, based on the first and second derivative of the function $w_{12}(z)$ with respect to $z$, a maximizer $\hat{z}_{12}^{*}$ for $w_{12}(z)$ is given by

$$
\hat{z}_{12}^{*}= \begin{cases}\operatorname{sol}\left\{z: F_{X}\left(\ell_{1, k_{1}}\right)=F_{X}(z)+z f_{X}(z), 2 f_{X}(z)+z f_{X}^{\prime}(z) \geq 0\right\} & \ell_{2, k_{2}} \leq z \leq \ell_{1, k_{1}} \\ \ell_{2, k_{2}} & \text { otherwise }\end{cases}
$$

The estimation $\hat{z}_{01}^{*}$ minimizing the distortion in region $\mathbf{Z}_{01}$ is

$$
\hat{z}_{01}^{*}= \begin{cases}\hat{z}_{11}^{*} & w_{11}\left(\hat{z}_{11}^{*}\right) \geq w_{12}\left(\hat{z}_{12}^{*}\right) \\ \hat{z}_{12}^{*} & \text { otherwise. }\end{cases}
$$

For the case $\left(U_{1}, U_{2}\right) \in \mathbf{Z}_{02} \subseteq \mathbf{Z}_{0}$ (i.e., $\left.\max \left(\ell_{1, k_{1}-1}, \ell_{2, k_{2}-1}\right)<\ell_{1, k_{1}} \leq \ell_{2, k_{2}}\right)$ we have that

$z \mathbb{P}\left(Z_{M} \geq z \mid X_{1} \in \mathcal{L}_{1, k_{1}}, X_{2} \in \mathcal{L}_{2, k_{2}}\right)= \begin{cases}z & z \leq \max \left(\ell_{1, k_{1}-1}, \ell_{2, k_{2}-1}\right) \\ z\left[1-F_{X \mid X \in \mathcal{L}_{1, k_{1}}}(z) F_{X \mid X \in \mathcal{L}_{2, k_{2}}}(z)\right] & \max \left(\ell_{1, k_{1}-1}, \ell_{2, k_{2}-1}\right) \leq z \leq \ell_{1, k_{1}} \\ z\left[1-F_{X \mid X \in \mathcal{L}_{2, k_{2}}}(z)\right] & \ell_{1, k_{1}} \leq z \leq \ell_{2, k_{2}} \\ 0 & z \geq \ell_{2, k_{2}}\end{cases}$

Since the maximum of each region can be also included on the boundary from $\max \left(\ell_{1, k_{1}-1}, \ell_{2, k_{2}-1}\right) \leq \hat{z} \leq \ell_{2, k_{2}}$, it is suffices to evaluate as follows,

$$
\hat{z}_{02}^{*}=\underset{z}{\arg \max } \begin{cases}w_{21}(z) & \max \left(\ell_{1, k_{1}-1}, \ell_{2, k_{2}-1}\right) \leq z \leq \ell_{1, k_{1}} \\ w_{22}(z) & \ell_{1, k_{1}} \leq z \leq \ell_{2, k_{2}}\end{cases}
$$

where

$$
\begin{aligned}
& w_{21}(z)=z\left(1-F_{X \mid X \in \mathcal{L}_{1, k_{1}}}(z) F_{X \mid X \in \mathcal{L}_{2, k_{2}}}(z)\right) \\
& w_{22}(z)=z\left(1-F_{X \mid X \in \mathcal{L}_{2, k_{2}}}(z)\right]
\end{aligned}
$$

Following a similar argument as above, a maximizer $\hat{z}_{21}^{*}$ of $w_{21}(z)$ is given by

$$
\hat{z}_{21}^{*}= \begin{cases}\operatorname{sol}\left\{z: w_{21}^{\prime}(z)=0, w_{21}^{\prime \prime}(z) \leq 0\right\} & \max \left(\ell_{1, k_{1}-1}, \ell_{2, k_{2}-1}\right) \leq z \leq \ell_{1, k_{1}} \\ \max \left(\ell_{1, k_{1}-1}, \ell_{2, k_{2}-1}\right) & \text { otherwise }\end{cases}
$$

and a maximizer $\hat{z}_{22}^{*}$ of $w_{22}(z)$ is given by

$$
\hat{z}_{22}^{*}= \begin{cases}\operatorname{sol}\left\{z: F_{X}\left(\ell_{2, k_{2}}\right)=F_{X}(z)+z f_{X}(z), 2 f_{X}(z)+z f_{X}^{\prime}(z) \geq 0\right\} & \ell_{1, k_{1}} \leq z \leq \ell_{2, k_{2}} \\ \ell_{1, k_{1}} & \text { otherwise. }\end{cases}
$$

The estimation $\hat{z}_{02}^{*}$ minimizing the distortion in region $\mathbf{Z}_{02}$ is

$$
\hat{z}_{02}^{*}= \begin{cases}\hat{z}_{21}^{*} & w_{21}\left(\hat{z}_{21}^{*}\right) \geq w_{22}\left(\hat{z}_{22}^{*}\right) \\ \hat{z}_{22}^{*} & \text { otherwise. }\end{cases}
$$

An expression for the distortion as a function of the quantizer parameters $\ell$ and the distribution $F(\cdot)$ is found by substituting in the Bayes estimator for in the different regions into 251]. 
9) Proof of Theorem 15. Proof: For a max and arg max quantizer, the average distortion is

$$
\begin{aligned}
\mathbb{E}\left[d_{M, A}\left(\left(X_{1}, X_{2}\right),(\hat{z}, \hat{i})\right)\right]= & \mathbb{E}\left[\mathbb{E}\left[d_{M, A}\left(\left(X_{1}, X_{2}\right),(\hat{z}, \hat{i})\right) \mid U_{1}, U_{2}\right]\right] \\
= & \sum_{k_{1}, k_{2}} \mathbb{E}\left[Z_{M}-\hat{z} \mathbb{1}_{\hat{z} \leq X_{\hat{\imath}}} \mid U_{1}=k_{1}, U_{2}=k_{2}\right] \mathbb{P}\left(U_{1}=k_{1}, U_{2}=k_{2}\right) \\
= & \sum_{\left(k_{1}, k_{2}\right) \in \mathbf{Z}_{1}} \mathbb{E}\left[X_{1}-\hat{z} \mathbb{1}_{\hat{z} \leq X_{\hat{i}}} \mid X_{1} \in \mathcal{L}_{1, k_{1}}, X_{2} \in \mathcal{L}_{2, k_{2}}\right] \mathbb{P}\left(X_{1} \in \mathcal{L}_{1, k_{1}}, X_{2} \in \mathcal{L}_{2, k_{2}}\right) \\
+ & \sum_{\left(k_{1}, k_{2}\right) \in \mathbf{Z}_{2}} \mathbb{E}\left[X_{2}-\hat{z} \mathbb{1}_{\hat{z} \leq X_{\hat{i}}} \mid X_{1} \in \mathcal{L}_{1, k_{1}}, X_{2} \in \mathcal{L}_{2, k_{2}}\right] \mathbb{P}\left(X_{1} \in \mathcal{L}_{1, k_{1}}, X_{2} \in \mathcal{L}_{2, k_{2}}\right) \\
+ & \sum_{\left(k_{1}, k_{2}\right) \in \mathbf{Z}_{0}} \mathbb{E}\left[Z_{M}-\hat{z} \mathbb{1}_{\hat{z} \leq X_{\hat{i}}} \mid X_{1} \in \mathcal{L}_{1, k_{1}}, X_{2} \in \mathcal{L}_{2, k_{2}}\right] \mathbb{P}\left(X_{1} \in \mathcal{L}_{1, k_{1}}, X_{2} \in \mathcal{L}_{2, k_{2}}\right)
\end{aligned}
$$

Similar to the case of max quantizer, to find a quantizer minimizing an average distortion, we need to evaluate a minimum distortion term as follows,

$$
\begin{aligned}
\underset{z, i}{\arg \min } \mathbb{E} & {\left[D_{M, A}\left(\left(Z_{M}, Z_{A}\right),(z, i)\right) \mid X_{1} \in \mathcal{L}_{1, k_{1}}, X_{2} \in \mathcal{L}_{2, k_{2}}\right] } \\
& =\underset{z, i}{\arg \min } \mathbb{E}\left[Z_{M}-z \mathbb{1}_{\left.X_{i} \geq z \mid X_{1} \in \mathcal{L}_{1, k_{1}}, X_{2} \in \mathcal{L}_{2, k_{2}}\right]}\right] \\
& =\underset{z, i}{\arg \max }\left\{z \mathbb{P}\left(X_{i} \geq z \mid X_{1} \in \mathcal{L}_{1, k_{1}}, X_{2} \in \mathcal{L}_{2, k_{2}}\right)\right\}
\end{aligned}
$$

When $\left(U_{1}, U_{2}\right) \in \mathbf{Z}_{1}$, then $Z_{M}=X_{1}$ and $Z_{A}=1$ and we take as our estimate $\hat{i}^{*}=1$ which gives

$$
\hat{z}_{1}^{*}=\underset{z}{\arg \max } z \mathbb{P}\left(X_{1} \geq z \mid X_{1} \in \mathcal{L}_{1, k_{1}}\right)
$$

In order to find an optimum estimation $\hat{z}_{1}^{*}$ minimizing the average distortion in region $\mathbf{Z}_{1}$, the necessary and sufficient condition is to find $z$ maximizing

$$
z \mathbb{P}\left(X_{1} \geq z \mid X_{1} \in \mathcal{L}_{1, k_{1}}\right)= \begin{cases}z & z \leq \ell_{1, k_{1}-1} \\ z \frac{F_{X}\left(\ell_{1, k_{1}}\right)-F_{X}(z)}{F_{X}\left(\ell_{1, k_{1}}\right)-F_{X}\left(\ell_{1, k_{1}-1}\right)} & z \in \mathcal{L}_{1, k_{1}} \\ 0 & z \geq \ell_{1, k_{1}}\end{cases}
$$

Since the maximum of each region can be included on the boundary from $\mathcal{L}_{1, k_{1}}$, it is suffices to evaluate as follows,

$$
\hat{z}_{1}^{*}=\underset{z \in \mathcal{L}_{1, k_{1}}}{\arg \max } z \frac{F_{X}\left(\ell_{1, k_{1}}\right)-F_{X}(z)}{F_{X}\left(\ell_{1, k_{1}}\right)-F_{X}\left(\ell_{1, k_{1}-1}\right)}
$$

Based on the first and second derivative with respect to $z$, the estimation $\hat{z}_{1}^{*}$ can be determined by

$$
\hat{z}_{1}^{*}= \begin{cases}\operatorname{sol}\left\{z: F_{X}\left(\ell_{1, k_{1}}\right)=F_{X}(z)+z f_{X}(z), 2 f_{X}(z)+z f_{X}^{\prime}(z) \geq 0\right\} & z \in \mathcal{L}_{1, k_{1}} \\ \ell_{1, k_{1}-1} & \text { otherwise }\end{cases}
$$

In order to find an average distortion in the region $\mathbf{Z}_{1}$, we first give $\mathbb{E}\left[X_{1} \mid X_{1} \in \mathcal{L}_{1, k_{1}}\right]$ as follows

$$
\mathbb{E}\left[X_{1} \mid X_{1} \in \mathcal{L}_{1, k_{1}}\right]=\int_{\ell_{1, k_{1}-1}}^{\ell_{1, k_{1}}} x \frac{f_{X}(x)}{F_{X}\left(\ell_{1, k_{1}}\right)-F_{X}\left(\ell_{1, k_{1}-1}\right)} \mathrm{d} x .
$$


The average minimum distortion when $\left(U_{1}, U_{2}\right) \in \mathbf{Z}_{1}$ is

$$
\begin{aligned}
\mathbb{E}\left[X_{1} \mid X_{1} \in \mathcal{L}_{1, k_{1}}\right]-\hat{z}_{1}^{*} \mathbb{P}\left(X_{1} \geq\right. & \left.\hat{z}_{1}^{*} \mid X_{1} \in \mathcal{L}_{1, k_{1}}\right)= \\
& \int_{\ell_{1, k_{1}-1}}^{\ell_{1, k_{1}}} x \frac{f_{X}(x)}{F_{X}\left(\ell_{1, k_{1}}\right)-F_{X}\left(\ell_{1, k_{1}-1}\right)} \mathrm{d} x-\hat{z}_{1}^{*} \frac{F_{X}\left(\ell_{1, k_{1}}\right)-F_{X}\left(\hat{z}_{1}^{*}\right)}{F_{X}\left(\ell_{1, k_{1}}\right)-F_{X}\left(\ell_{1, k_{1}-1}\right)}
\end{aligned}
$$

When $\left(U_{1}, U_{2}\right) \in \mathbf{Z}_{2}$, then $Z_{M}=X_{2}$ and $Z_{A}=2$ and we take as our estimate $\hat{i}^{*}=2$ which gives

$$
\hat{z}_{2}^{*}=\underset{z}{\arg \max } z \mathbb{P}\left(X_{2} \geq z \mid X_{2} \in \mathcal{L}_{2, k_{2}}\right)
$$

Similar to the region $\mathbf{Z}_{1}$, the estimation $\hat{z}_{2}^{*}$ can be determined by the solution as follows,

$$
\hat{z}_{2}^{*}= \begin{cases}\operatorname{sol}\left\{z: F_{X}\left(\ell_{2, k_{2}}\right)=F_{X}(z)+z f_{X}(z), 2 f_{X}(z)+z f_{X}^{\prime}(z) \geq 0\right\} & z \in \mathcal{L}_{2, k_{2}} \\ \ell_{2, k_{2}-1} & \text { otherwise }\end{cases}
$$

The average minimum distortion when $\left(U_{1}, U_{2}\right) \in \mathbf{Z}_{2}$ is

$$
\begin{aligned}
\mathbb{E}\left[X_{2} \mid X_{2} \in \mathcal{L}_{2, k_{2}}\right]-\hat{z}_{2}^{*} \mathbb{P}\left(X_{2} \geq\right. & \left.\hat{z}_{2}^{*} \mid X_{2} \in \mathcal{L}_{2, k_{2}}\right)= \\
& \int_{\ell_{2, k_{2}-1}}^{\ell_{2, k_{2}}} x \frac{f_{X}(x)}{F_{X}\left(\ell_{2, k_{2}}\right)-F_{X}\left(\ell_{2, k_{2}-1}\right)} \mathrm{d} x-\hat{z}_{2}^{*} \frac{F_{X}\left(\ell_{2, k_{2}}\right)-F_{X}\left(\hat{z}_{2}^{*}\right)}{F_{X}\left(\ell_{2, k_{2}}\right)-F_{X}\left(\ell_{2, k_{2}-1}\right)}
\end{aligned}
$$

When $\left(U_{1}, U_{2}\right) \in \mathbf{Z}_{0}$, then $Z_{M}$ could be either $X_{1}$ or $X_{2}$ and it suffices to compare

$$
z \mathbb{P}\left(Z_{M} \geq z \mid X_{1} \in \mathcal{L}_{1, k_{1}}, X_{2} \in \mathcal{L}_{2, k_{2}}\right)
$$

under $Z_{M}=X_{1}$ and $Z_{M}=X_{2}$. We need to find $\hat{z}^{*}$ and $\hat{i}^{*}$ as follows,

$$
\underset{z, i}{\arg \max } z \frac{F_{X}\left(\ell_{i, k_{i}}\right)-F_{X}(z)}{F_{X}\left(\ell_{i, k_{i}}\right)-F_{X}\left(\ell_{i, k_{i}-1}\right)}
$$

An expression for the distortion as a function of the quantizer parameters $\ell$ and the distribution $F(\cdot)$ is found by substituting in the Bayes estimator for in the different regions into 280 . 